\title{
Star formation quenching stages of active and non-active galaxies
}

\author{
V. Kalinova ${ }^{1}$, D. Colombo ${ }^{1}$, S. F. Sánchez ${ }^{2}$, K. Kodaira ${ }^{1,3,4}$, R. García-Benito ${ }^{5}$, R. González Delgado ${ }^{5}$, \\ E. Rosolowsky ${ }^{6}$, and E. A. D. Lacerda ${ }^{2}$ \\ 1 Max Planck Institute for Radioastronomy, Auf dem Hügel 69, 53121 Bonn, Germany \\ e-mail: kalinova@mpifr.de \\ 2 Instituto de Astronomía, Universidad Nacional Autonóma de México, A.P. 70-264, 04510 Mexico DF, Mexico \\ 3 National Astronomical Observatory of Japan, Osawa2-21-1, Mitaka-shi, 181-8588 Tokyo, Japan \\ ${ }^{4}$ SOKENDAI, International Village, Hayama-machi, Miura-gun 240-0193, Kanagawa-ken, Japan \\ 5 Instituto de Astrofísica de Andalucía, CSIC, Apartado de correos 3004, 18080 Granada, Spain \\ 6 Department of Physics 4-181 CCIS, University of Alberta, Edmonton, AB T6G 2E1, Canada
}

Received 12 November 2020 / Accepted 21 January 2021

\begin{abstract}
The mechanisms that bring galaxies to strongly reduce their star formation activity (star-formation quenching) remain poorly understood. To better study galaxy evolution, we propose a classification based on maps of ionised hydrogen distribution traced by the kiloparsec-resolved, equivalent width of $\mathrm{H} \alpha$ maps, and the nuclear activity of the galaxies using information from the BaldwinPhilips-Terlevich diagnostic diagrams. Using these tools, we group a sample of 238 galaxies from the Calar Alto Legacy Integral Field spectroscopy Area survey in six quenching stages (QSs): (i) objects dominated by recent star formation; (ii) systems that present a quiescent-nuclear-ring structure in their centre; (iii) galaxies that are centrally quiescent; (iv) galaxies with no clear pattern in their ionisation gas distribution (mixed); (v) systems that posses only a few star-forming regions (nearly retired), or (vi) galaxies that are completely quiescent (fully retired). Regarding their nuclear activity, we further divide the galaxies into two groups: active systems that host a weak or strong active galactic nucleus (AGN) at their centre, and non-active objects. Galaxies grouped into quenchingstage classes occupy specific locations on the star-formation-rate versus stellar mass diagram. The 'blue cloud' is populated by the star-forming and the quiescent-nuclear-ring galaxies, the 'green valley' is populated by centrally quiescent and mixed systems, and the 'red sequence' by the nearly- and fully retired objects. Generally, galaxies that host a weak or strong AGN show properties comparable to those of their non-active counterparts at the same QSs, except for the AGN-hosting star-forming systems. The degree of star-formation quenching increases along the present emission-line pattern sequence from star-forming to fully retired. The proposed emission-line classes reinforce the 'inside-out' quenching scenario, which foresees that the suppression of star formation begins from the central regions of the galaxies.
\end{abstract}

Key words. galaxies: evolution - galaxies: structure - galaxies: star formation - galaxies: active - galaxies: nuclei galaxies: fundamental parameters

\section{Introduction}

Distributions of galaxy properties such as morphologies (Hubble 1926), colours (Strateva et al. 2001; Baldry et al. 2004), star-formation rates (SFRs; Brinchmann et al. 2004; Renzini \& Peng 2015), ages (Gallazzi et al. 2005, 2008; Zibetti et al. 2017), and gas content (e.g. Young \& Scoville 1991; Blanton \& Moustakas 2009) in the local universe show strong bi-modality. Similar bi-modality is observed even up to $z \sim 2.5$ (Brammer et al. 2009; Williams et al. 2009). The so-called red sequence is populated by red spheroidal systems, while the blue cloud hosts blue disc galaxies. The region in between is called the green valley and is underpopulated, suggesting that the transition occurs within a narrow range of certain galaxy parameters from the blue cloud to the red sequence. Galaxies in the green valley do not show a particular morphology (Schawinski et al. 2014) or other particular properties, rather their colour is the consequence of the switching off of star formation, a process usually referred to as star formation quenching (e.g. Faber et al. 2007). Indeed, when posed on an SFR versus stellar mass $\left(M_{*}\right)$ diagram, galaxies show the same bi-modality, with star-forming galaxies tightly organised across the star formation main sequence (SFMS, e.g. Brinchmann et al. 2004; Whitaker et al. 2012; Renzini \& Peng
2015; Speagle et al. 2014; Cano-Díaz et al. 2016; Sánchez et al. 2019), retired galaxies in the corresponding red sequence, and galaxies in the process of quenching (immediately below the SFMS) in the green valley (e.g. Schawinski et al. 2014).

The integrated SFR versus $M_{*}$ diagram $\left(\mathrm{SFR}-M_{*}\right)$ has been broadly explored in the literature (e.g. Schawinski et al. 2014; Renzini \& Peng 2015; Catalán-Torrecilla et al. 2015, 2017; González Delgado et al. 2016; Cano-Díaz et al. 2016, 2019; Belfiore et al. 2018; Bluck et al. 2019; Sánchez et al. 2018, 2019; Lacerda et al. 2020) and has served as an important diagnostic tool for studying galaxy evolution.

Additionally, galaxy evolution theories have been largely constrained through ionised gas emission-line (EL) classification schemes that study the star formation, chemical properties, and nuclear activity of large samples of galaxies. Baldwin et al. (1981; see also Veilleux \& Osterbrock 1987) originally proposed a diagram (now known as the Baldwin-Philips-Terlevich (BPT) diagram) that uses the intensity ratio of emission lines ([O III] $\lambda 5007 / \mathrm{H} \beta$ versus [N II] $\lambda 6584 / \mathrm{H} \alpha$ line ratios) to distinguish between dominant excitation sources (such as HII regions, power-law continuum spectrum photo-ionisations, and heating by shock waves in the original formulation) from the spectra of extragalactic objects. Later on, Kauffmann et al. (2003) defined 
loci on the BPT diagram to distinguish star-forming and starburst galaxies (see also Kewley et al. 2001), Seyfert regions related to galaxies hosting an active galactic nucleus (AGN), or low-ionisation nuclear emission regions (LINERs), in which the ionisation might be attributed to old and hot stars (e.g. Stasińska et al. 2008).

More recently, Cid Fernandes et al. (2011) proposed a different bi-dimensional diagram to disentangle the excitation source in the centre of galaxies: The 'WHAN' diagram considers the equivalent width of the $\mathrm{H} \alpha\left(W_{\mathrm{H} \alpha}\right)$ versus the [N II] $\lambda 6584 / \mathrm{H} \alpha$ ratio to classify galaxies into purely star-forming or strong AGN hosts. Additionally, this diagnostic is able to distinguish between objects that are retired (or passive) and galaxies that genuinely host a weak AGN.

The advent of spatially resolved integral field spectroscopy (IFS) surveys such as ATLAS ${ }^{3 \mathrm{D}}$ (Cappellari et al. 2011), Calar Alto Legacy Integral Field spectroscopy Area (CALIFA; Sánchez et al. 2012), Sydney-AAO Multi-object Integral field spectrograph (SAMI; Croom et al. 2012), Mapping Nearby Galaxies at Apache Point Observatory (MaNGA; Bundy et al. 2015), and All-weather MUse Supernova Integral field Nearby Galaxies (AMUSING++; López-Cobá et al. 2020) has provided large statistical samples with which to study excitation sources of the ionised gas not only between galaxies, but also within them. In particular, they enable identification of the dominant excitation source of a galaxy, because different galactic regions can be characterised by a large variety of ionisation effects (see Sánchez 2020 for an extensive review).

An attempt to classify single regions of galaxies through the use of the BPT diagram was made by Singh et al. (2013), who show that excitation properties that characterise LINERs are not simply limited to the nuclear regions, but extend far away from the galactic centre. Those 'low ionisation emission regions' (therefore redefined as 'LIERs') are probably due to post-AGB stars rather than to AGN activity. Following a similar method, Belfiore et al. (2016) used the BPT scheme to group galaxies based on their dominant excitation source and consider the spatially resolved patterns described by the diagram. This classification distinguishes between galaxies dominated by star formation, galaxies hosting an AGN, objects that show LIERs only in the centre (cLIERs) or throughout their full extent (eLIERs), and mergers. In particular, cLIERs are bulged spiral galaxies located in the green valley, while eLIERs are basically elliptical, retired objects.

The diffuse ionised gas classification proposed by Lacerda et al. (2018) instead put emphasis on the use of resolved $W_{\mathrm{H} \alpha}$ maps to show where the gas excitation in the different galaxy regions is powered by hot low-mass evolved stars (HOLMES; Flores-Fajardo et al. 2011), HII regions (and therefore star formation), or a mixture of both effects. This scheme is simple, because it involves the use of $W_{\mathrm{H} \alpha}$ only, but effective because its predictions are largely consistent with those based on the BPT diagram. However, it does not aim to put a galaxy in a given category, but rather to classify galactic regions in diffuse ionised gas classes. Furthermore, it does not allow the user to distinguish excitation due to AGNs. Recent works of Sánchez et al. (2018) and Lacerda et al. (2020) define and explore the properties of AGN hosts from large samples of MaNGA and CALIFA galaxies, respectively, through classification based on the information from the BPT diagrams and the $W_{\mathrm{H} \alpha}$ values of the central galaxy regions.

In the present article, we synthesise the $W_{\mathrm{H} \alpha}$ values and BPT-diagram classification approaches in a similar way in order to propose a new bi-dimensional method that distinguishes between several 'star formation quenching stages', considering the nuclear activity of the galaxy. The novelty of our method is that it not only defines galaxy groups based on the global $W_{\mathrm{H} \alpha}$ value or the $W_{\mathrm{H} \alpha}$ value at particular radii, but also considers the patterns described by the resolved $W_{\mathrm{H} \alpha}$ map across the entire galaxy. Our method is inspired by the previous work of Sánchez et al. (2013), who first proposed the use of the resolved $W_{\mathrm{H} \alpha}$ map to distinguish between star-forming and retired regions in the galaxies, extending the selection introduced by the WHAN diagram (see also Sánchez et al. 2014, 2018; Sánchez-Menguiano et al. 2016, 2018; Cano-Díaz et al. 2016, 2019; López-Cobá et al. 2019; Sánchez 2020). The proposed classification compares the properties of active and non-active systems with various ionisation distributions and is used here to investigate the quenching mechanisms that transform galaxies from one type to another.

This study is organised as follows: Sect. 2 describes the sample selection and the analysed data; Sect. 3 defines the criteria of the emission-line classification; and Sects. 4 and 5 present the resolved and global properties of the EL galaxies. Finally, Sects. 6 and 7 present our discussion and summary, respectively.

\section{Data and analysis}

\subsection{Sample selection}

Our study is based on CALIFA integral-field unit (IFU) data, which observed 667 galaxies (in its third data release, Sánchez et al. 2016a) in the redshift range $0.005<z<0.03$ using the Potsdam Multi-Aperture Spectrophotometer (PMAS, Roth et al. 2005) in PPaK (PMAS fiber Package, Verheijen et al. 2004; Kelz et al. 2006) mode, installed on the $3.5 \mathrm{~m}$ telescope at the Calar Alto observatory. In particular, CALIFA targets span various morphologies and stellar masses, forming a representative sample of nearby Universe galaxies. Further details can be found in the survey-presentation paper (Sánchez et al. 2012), the datarelease papers (Husemann et al. 2013; García-Benito et al. 2015; Sánchez et al. 2016a), and the sample paper of Walcher et al. (2014).

For this work, we adopt the sample of 238 CALIFA galaxies originally explored in Kalinova et al. (2017a, hereafter K17). This sample was originally selected from Falcón-Barroso et al. (2017), who provided stellar kinematics maps for 300 CALIFA galaxies observed until June 2014. After exclusion of mergers and galaxies with unreliable dynamical models, K17 present a sample of 238 targets in a broad range of stellar masses (from $6 \times 10^{8} M_{\odot}$ to $5 \times 10^{11} M_{\odot}$ ) and types (from E1 to $\mathrm{Sdm}$ ). The current sample is representative of the CALIFA mother sample (see Fig. 1 in K17) and is therefore representative of the local Universe galaxy population too.

\subsection{Emission-line data}

There are three sets of CALIFA data: low-spectral-resolution mode V500 ( $F W H M \sim 6 \AA)$, high-resolution V1200 (FWHM $2.3 \AA$ ), and a combination of both observation setups, called COMBO data $(F W H M \sim 6 \AA)$. V500 ranges between 3745 and $7500 \AA$, V1200 between 3650 and $4840 \AA$, and COMBO between 3700 and $7500 \AA$ (see Sánchez et al. 2016a). Depending on scientific goals, we use different data sets in order to optimise our results. For example, the COMBO dataset has the same resolution as V500, but gives more reliable spectrophotometry at the blue end of its spectra $(\lambda<4600 \AA)$, which contains important stellar population tracers (i.e. COMBO data are not influenced 
by vignetting effects). For this set of galaxies, we make use of the ionised gas maps of $\mathrm{H} \alpha, \mathrm{H} \beta,[\mathrm{NII}]$, [SII], [OI], [OIII] line fluxes, and $W_{\mathrm{H} \alpha}$ obtained with the Pipe $3 \mathrm{D}$ pipeline using the low-resolution mode V500 of the CALIFA data set, where the lines are strong and present in this spectral window (in contrast to V1200 data), and available for all galaxies of our sample (in contrast to COMBO data).

Full details about Pipe3D are given in Sánchez et al. (2016c,b); here we provide a short description. The pipeline uses a combination of synthetic simple stellar population (SSP) spectra from the GRANADA library (Martins et al. 2005) and empirical ones from the MILES project (Sánchez-Blázquez et al. 2006; Vazdekis et al. 2010; Falcón-Barroso et al. 2011). This joint SSPlibrary (see more details in Cid Fernandes et al. 2013) adopts the Salpeter (Salpeter 1955) initial mass function (IMF). It consists of 156 templates, covering 39 stellar ages (from $1 \mathrm{Myr}$ to $14 \mathrm{Gyr})$, and 4 metallicities ranges $\left(Z / Z_{\odot}=0.2,0.4,1\right.$ and 1.5$)$. The library uses Girardi et al. (2000) evolutionary tracks, except for the youngest ages between $1 \mathrm{Myr}$ and $3 \mathrm{Myr}$, which are based on Geneva tracks (Schaller et al. 1992; Schaerer et al. 1993a,b; Charbonnel et al. 1993).

The original $V$-band data-cube is binned to increase the signal-to-noise ratio $(\mathrm{S} / \mathrm{N})$ but keep the overlap between regions with different physics to a minimum. A spaxel-wise stellar population fit is performed using Fit3D. Afterwards, the model is evaluated by considering the continuum flux within each spatial bin. The ionised emission line data cube is then constructed by subtracting the stellar-population model spaxel by spaxel. Pipe3D analyses 52 emission lines and provides various maps (e.g. flux intensity, velocity, velocity dispersion and $W_{\mathrm{H} \alpha}$ ), alongside their uncertainties.

\subsection{Stellar population data}

In order to derive the star-formation history properties of the galaxies and to optimise our results, we rely on the COMBO dataset, which provides a free vignetted field of view (FOV) on the blue side of the spectral range in contrast to V500 alone (although COMBO data are not available for seven galaxies in our sample; see Table B.1). We estimated the stellar surfacemass-density $\left(\mu_{*}\right)$, stellar mass $\left(M_{*}\right)$, age $(\tau)$, metallicity $(Z)$, and SFR of the galaxies assuming a Chabrier IMF (Chabrier 2003), which is necessary for the comparison of our sample with Sloan Digital Sky Survey $\left(\mathrm{SDSS}^{1}\right)$ reference galaxies in the context of SFR-M* diagram exploration in Sect. 6.1. As PIPE3D only adopts a Salpeter IMF, this makes it inconvenient for our analysis here. We instead run the STARLIGHT spectral synthesis code of Cid Fernandes et al. (2005) on the COMBO data, fitting the individual spectra for each spatially resolved element of the IFU, and then implementing the output results in the platform of Python CALIFA STARLIGHT Synthesis organiser (PyCASSO; de Amorim et al. 2017) to obtain the spatially resolved data.

For the fits, we use the $\mathrm{CBe}$ base of Charlot \& Bruzual (2007, priv. comm.), which is a combination of 246 single stellar population (SSP) spectra covering 41 ages from 0.001 to $14 \mathrm{Gyr}$ and six metallicity ranges: $\log Z / Z_{\odot}=-2.3,-1.7$, $-0.7,-0.4,0,+0.4$. The SSP models are revised from Bruzual \& Charlot (2003), where both MILES (Sánchez-Blázquez et al. 2006; Falcón-Barroso et al. 2011) and GRANADA (Martins et al. 2005) stellar libraries replace the original library STELIB (Le Borgne et al. 2003). Further, 'Padova 1994' stellar tracks has been applied (Alongi et al. 1993; Bressan et al. 1993; Fagotto

\footnotetext{
1 https: //WWW. sdss.org/
}

et al. 1994a,b,c; Girardi et al. 1996). For more details about the SSP analysis, we refer to the related works of Cid Fernandes et al. (2013), González Delgado et al. (2015), de Amorim et al. (2017), García-Benito et al. (2017, 2019).

The mean stellar surface brightness, $\mu_{*}$, is extinction corrected and calculated within $1 R_{\mathrm{e}}$. The galaxy age $(\tau)$ and metallicity $(Z)$ are light- and mass-weighted within $1 R_{\mathrm{e}}$, respectively. The total SFR is calculated from the spatially resolved star formation history of galaxies by adding the amount of stellar mass formed into stars in the last $32 \mathrm{Myr}$ (and divided by this time interval; González Delgado et al. 2016, 2017; de Amorim et al. 2017). This approach helps to account for the star formation of the galaxies that is heavily obscured or has a low quantity of ionised gas (i.e. those galaxies where the $\mathrm{H}_{\alpha}$ emission is very weak). The SFR from $\mathrm{H}_{\alpha}$ has a shorter timescale of emission in comparison to the SFR from SSP, which mainly comes from OB stars (3 Myr, Kennicutt \& Evans 2012). In addition, the SSPbased approach averages over stochastic star formation to provide a good estimation of the SFR.

\subsection{Photometric data}

We analysed the photometric properties of our galaxies using SDSS data (Abazajian et al. 2009; Alam et al. 2015). We derived the bulge-to-disc (B/D) ratios of the galaxies from their bulgeto-total $(\mathrm{B} / \mathrm{T})$ and disc-to-total $(\mathrm{D} / \mathrm{T})$ flux ratios, which are provided by Méndez-Abreu et al. (2017), who analyse $r$-band SDSS images from Data Release (DR) 7 (Abazajian et al. 2009) of 404 CALIFA galaxies. The overlap with our sample (for galaxies with both available $\mathrm{B} / \mathrm{T}$ and $\mathrm{D} / \mathrm{T}$ ratios) is 127 galaxies, which approximately covers half of the galaxies within each EL class, with no significant changes across our classes. Thus, we do not expect large bias in our conclusions regarding B/D ratios.

We calculated the total luminosity $\left(L_{\mathrm{r}}^{\text {tot }}\right)$ of the galaxies from the $r$-band SDSS (DR12; Alam et al. 2015) images using the Multi-Gaussian Expansion method (MGE; Emsellem et al. $1994)$ by $\mathrm{K} 17$. We measured the effective radii $\left(R_{\mathrm{e}}\right)$ using a growth curve analysis based on elliptical apertures from SDSS DR7 (Abazajian et al. 2009) images, which provide reliable estimations in cases of highly inclined galaxies (Sanchez et al., in prep.). The galaxy morphology and the presence of a bar were defined visually by a few members of the CALIFA team as described in Walcher et al. (2014).

\subsection{Kinematical and dynamical data}

To calculate the galactic specific angular momentum $\left(\lambda_{R_{\mathrm{e}}}\right)$ of our galaxies, we use the public available stellar kinematic maps of the CALIFA galaxies, provided by Falcón-Barroso et al. (2017). They use the high-resolution V1200 setup of CALIFA data, which has much higher spectral resolution $\left(\sim 72 \mathrm{~km} \mathrm{~s}^{-1}\right)$ than V500 grating $\left(\sim 139 \mathrm{~km} \mathrm{~s}^{-1}\right)$, and where V500 has difficulty in reliably measuring velocity dispersions below $100 \mathrm{~km} \mathrm{~s}^{-1}$ (see Sect. 4.2 of their paper). Further, Falcón-Barroso et al. (2017) derive the line-of-sight velocity $(V)$ and velocity dispersion $(\sigma)$ distributions after fitting the stellar continuum of the galaxies in each spatial bin using the pPXF code of Cappellari \& Emsellem (2004) with stellar templates from the Indo-US spectral library (Valdes et al. 2004). The averaging of the pixels was done using the Voronoi 2D binning method of Cappellari \& Copin (2003) with a minimal $\mathrm{S} / \mathrm{N}$ of 20 per pixel.

We calculate the specific angular momentum $\lambda_{R_{\mathrm{e}}}$ using Eq. (6) in Emsellem et al. (2007): 


$$
\lambda_{R_{\mathrm{e}}}=\frac{\sum_{i=0}^{N_{k}} F_{i} R_{i}\left|V_{i}\right|}{\sum_{i=0}^{N_{k}} F_{i} R_{i} \sqrt{V_{i}^{2}+\sigma_{i}^{2}}},
$$

where $F_{i}, R_{i}, V_{i}$ and $\sigma_{i}$ are the flux, circular radius, velocity, and velocity dispersion of the $i$-th spatial bin.

We report $\lambda_{R_{\mathrm{e}}}$ as the integrated value within one effective radius of the galaxy, reached by all of our targets. Our measurements of $\lambda_{R_{\mathrm{e}}}$ are comparable to the published calculations of Falcón-Barroso et al. (2019), although we adopt different values for the position angles and the inclinations of the galaxies (from K17).

The amplitude $\left(V_{\mathrm{c}, \max }\right)$ of the circular velocity curve of the galaxies within $1.5 R_{\mathrm{e}}$ is inferred from stellar dynamical modelling (Sect. 3.1 in K17) based on axisymmetric Jeans equations (i.e. using the $\mathrm{JAM}^{2}$ code of Cappellari 2008). In addition, the total dynamical mass $M_{\text {dyn }}^{\text {tot }}$ (panel K) comes from multiplication of the total luminosity $\left(L_{r}^{\text {tot }}\right)$ derived via the MGE method (Emsellem et al. 1994) by the dynamical mass-to-light ratio $\gamma_{\text {dyn }}$ of the galaxies obtained using the Markov Chain Monte Carlo approach (MCMC; Foreman-Mackey et al. 2013) of the JAM model (see Kalinova et al. 2017b). Our masses are comparable to the recent calculations of the dynamical mass by Aquino-Ortíz et al. (2018), who use the kinematic parameter $S_{k}^{2}=K V_{\text {rot }}^{2}+\sigma^{2}$ as a dynamical proxy (where $K$ is constant, $V_{\text {rot }}$ is the rotation velocity, and $\sigma$ is the velocity dispersion of the galaxy; Weiner et al. 2006). To calibrate the constant $K$, Aquino-Ortíz et al. (2018) use the dynamical mass measurements of Leung et al. (2018), Zhu et al. (2018a), and Zhu et al. (2018b).

Through $M_{\text {dyn }}^{\text {tot }}$ and $M_{*}^{\text {tot }}$, we calculate the mass discrepancy factor $f_{d}=1-\left(M_{*}^{\text {tot }} / M_{\mathrm{dyn}}^{\mathrm{tot}}\right)$, showing the relationship between the total stellar and dynamical masses as a fraction.

\section{Two-dimensional emission-line classification}

We classify galaxies according to their ionisation, following recent studies in the field (Schawinski et al. 2007; Stasińska et al. 2008; Cid Fernandes et al. 2011; Singh et al. 2013; Belfiore et al. 2016; Sánchez et al. 2018; Sánchez 2020; Lacerda et al. 2020). In particular, our classification is based on the peculiar patterns defined by the spatially resolved $\mathrm{H} \alpha$-equivalent-width $\left(W_{\mathrm{H} \alpha}\right)$ value within the galaxies (similar to Cid Fernandes et al. 2011; Sánchez et al. 2018; Lacerda et al. 2018, 2020; EspinosaPonce et al. 2020). Briefly, star-forming or HII regions show $W_{\mathrm{H} \alpha}>6 \AA$; finding $W_{\mathrm{H} \alpha} \leq 3 \AA$ indicates that the gas in the region is ionised by the old stellar population (post-AGB stars or HOLMES); values of $3 \AA<W_{\mathrm{H} \alpha} \leq 6 \AA$ are consistent neither with star formation nor HOLMES, but rather with other mixed phenomena (e.g. diffuse ionised gas, high-velocity shocks; e.g. Lacerda et al. 2018). Therefore the $W_{\mathrm{H} \alpha}$ values of $3 \AA$ and $6 \AA$ mark the thresholds we use to recognise patterns in the resolved $W_{\mathrm{H} \alpha}$ map (see also Cid Fernandes et al. 2011).

To distinguish between the active and non-active galaxies of our sample (i.e. whether or not they host an AGN in their centres), we use three BPT excitation diagnostic diagrams (and their corresponding maps) involving the [OIII], [SII], [OI], and [NII] line ratios with respect to the Balmer lines (Baldwin et al. 1981, Veilleux \& Osterbrock 1987). We assess these diagrams using the loci separating different ionisation regimes proposed by Kauffmann et al. (2003). The utility of combining $W_{\mathrm{H} \alpha}$ together with the BPT diagnostic diagrams has been suggested by many

\footnotetext{
2 http://purl.org/cappellari/software
}

authors (e.g. Sarzi et al. 2010; Cid Fernandes et al. 2010; Singh et al. 2013; Sánchez et al. 2014; Lacerda et al. 2018, 2020). Thus, the galaxy is defined as a host of a weak AGN if its central pixels, located within $0.5 R_{\mathrm{e}}$ of the galaxy, populate the Seyfert region of the BPT diagrams and their $W_{\mathrm{H} \alpha}$ values range between $3 \AA$ and $6 \AA$. In case the $W_{\mathrm{H} \alpha}$ values of those central pixels go above $6 \AA$, then the galaxy is considered to host a strong AGN (see Sect. 3.2 for more details.)

We apply a 1- $\sigma$ clipping to the $W_{\mathrm{H} \alpha}$ maps and measurements in the BPT diagrams, which removes low-signal-to-noise-ratio spaxels. We experimented with a stricter 3- $\sigma$ clipping, but this omitted targets with lower S/Ns in the emission-line data (typically for old population galaxies).

Visually identifying dominant patterns in the $W_{\mathrm{H} \alpha}$ maps of CALIFA galaxies, we find six quenching stages (QSs): star-forming, quiescent-nuclear-ring, centrally quiescent, mixed, nearly retired, and fully retired. On the other hand, from the BPT diagrams we distinguish three nuclear activities (NAs) of the galaxies: non-active, weak AGN (wAGN), and strong AGN (sAGN).

If we explore the properties of the galaxies only by the QS criteria, we refer to a 'quenching classification', while if we explore the galaxies only through their nuclear activity criteria, we link to a 'nuclear activity classification'. In Fig. 1, we combine both QS and NA criteria and define a two-dimensional (2D) emission-line classification called QuestNA (QUEnching STages and Nuclear Activity).

QuestNA can be expressed as a matrix of six QS rows by three NA columns, giving eighteen EL classes, but in reality the EL classes only show fourteen populated classes, because centrally quiescent and fully retired QSs do not allow a nuclear activity in the centre of the galaxies by definition: the $W_{\mathrm{H} \alpha}$ values in these regions are equal to or below $3 \AA$, which is too low to distinguish the AGN activity from the other processes in the galaxies (e.g. Cid Fernandes et al. 2010; Sánchez et al. 2014; Lacerda et al. 2020). The QS rows are ordered by increasing ratio of retired/star-forming regions in the galaxy, while the NA columns are organised according to the increasing nuclear activity of the galaxy (i.e. non-active $\rightarrow \mathrm{wAGN} \rightarrow \mathrm{sAGN}$ ).

\subsection{Quenching stage criteria}

Based on the distribution of ionisation in different regions within the 238 CALIFA galaxies and visual inspection of their $W_{\mathrm{H} \alpha}$ maps, we propose the following QSs of the galaxies (i.e. this is the first dimension of the emission-line classification):

(i) Star-forming galaxies. The first row of panels $(\mathrm{A}-\mathrm{C})$ of Fig. 1 exemplifies the typical star-forming class galaxies (NGC 1151, NGC 0214 and NGC 7466) from each type of nuclear activity (non-active, wAGN, and sAGN, respectively). The median value of the $W_{\mathrm{H} \alpha}$ spaxels should be larger than $6 \AA$ and there are no large regions in these galaxies with lowionisation emission lines, where $W_{\mathrm{H} \alpha}<3 \AA$ (such low ionisation spaxels are usually less than $3 \%$ of the total ones). The starforming class can be represented by both active and non-active galaxies.

(ii) Quiescent-nuclear-ring galaxies. The second row of panels (D-F) of Fig. 1 represents the quiescent-nuclear-ring class galaxies (NGC 3811, NGC 7321 and UGC03995) from each type of nuclear activity. This group is characterised by a quiescent-nuclear-ring region within the $0.2-0.5 R_{\mathrm{e}}$ of the galaxy, where the $W_{\mathrm{H} \alpha}$ is below $3 \AA$. On the other hand, the 


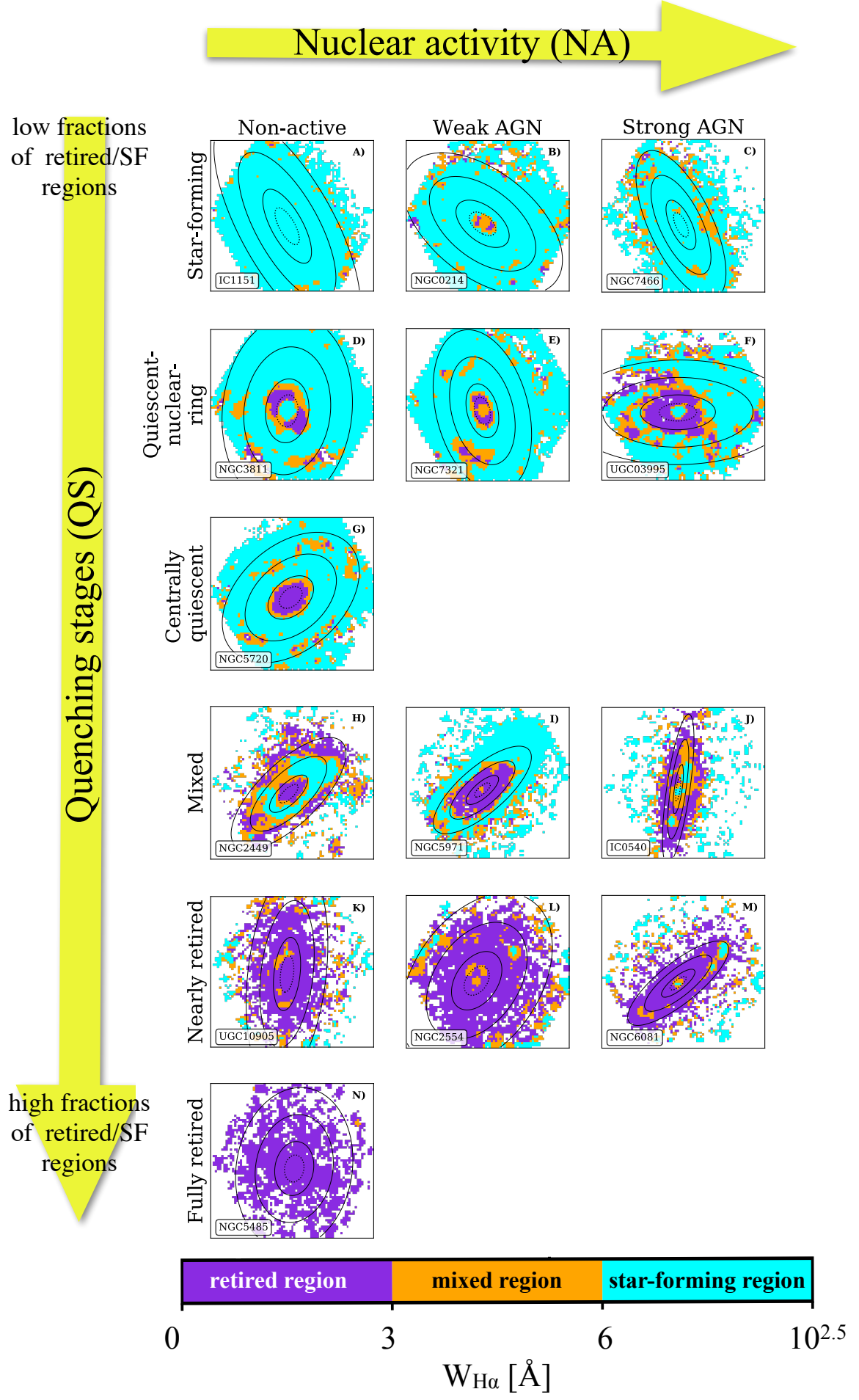

Fig. 1. Two-dimensional emission line classification 'QuestNA' of our sample of galaxies (see Sect. 3). The rows represent the quenching stages of the galaxies: star-forming, quiescent-nuclear-ring, centrally quiescent, mixed, nearly retired, and fully retired with increasing 'retired/star-forming regions' ratio of the galaxy from top to bottom. The columns reflect the level of galaxy nuclear activity: non-active, weak, and strong AGN from left to right. Each panel of the scheme shows the $W_{\mathrm{H} \alpha}$ map of the galaxy, where the dashed contour indicates 0.5 of the effective radius, and the continuous contours correspond to 1,2 , and $3 R_{\mathrm{e}}$, respectively. The colour bar of the $W_{\mathrm{H} \alpha}$ maps divides the regions of the galaxy into star-forming ( $\left.W_{\mathrm{H} \alpha}>6 \AA\right)$, retired $\left(W_{\mathrm{H} \alpha} \leq 3 \AA\right)$, and mixed $\left(3 \AA<W_{\mathrm{H} \alpha} \leq 6 \AA\right)$. There are 14 emission-line classes of QuestNA instead of 18, because two of the quenching stages, centrally quiescent and fully retired, are only non-active galaxies, which by definition are fully retired in the central regions. central spaxels, which are surrounded by the ring, always have $W_{\mathrm{H} \alpha}$ values above $3 \AA$. In the outer regions of the galaxies $(0.5-$ $2.0 R_{\mathrm{e}}$ ), there is evidence for the presence of a star-forming disc with $W_{\mathrm{H} \alpha}$ values going above $6 \AA$. The quiescent-nuclear-ring class contains both active and non-active galaxies.

(iii) Centrally quiescent galaxies. In panel G of Fig. 1, galaxy NGC 5720 represents the centrally-quiescent class galaxies, which shows low-ionisation EL regions at small galactocentric radii: $W_{\mathrm{H} \alpha}$ stays below $3 \AA$ up to $0.5 R_{\mathrm{e}}$. There is evidence for the presence of a star-forming disc because $W_{\mathrm{H} \alpha}$ values go above $6 \AA$ within the range $0.5-2.0 R_{\mathrm{e}}$. The centrally quiescent class are only non-active galaxies because the $W_{\mathrm{H} \alpha}$ value of the central pixels is always equal to or below $3 \AA$ even in cases where the line ratios populate the Seyfert region of the BPT diagrams. This class is similar to the cLIER galaxies from the [SII]-BPT classification of Belfiore et al. (2016).

(iv) Mixed galaxies. The fourth row of panels $(\mathrm{H}, \mathrm{I}$ and J) of Fig. 1 exemplifies the mixed galaxies (NGC 2449, NGC 5971 and IC0540). This class is characterised by starforming regions (high-ionisation EL regions, where $W_{\mathrm{H} \alpha}>6 \AA$ ), 


\section{Non-active galaxy: BPT diagram $+\mathrm{W}_{\mathrm{H} \alpha}$ criterion}
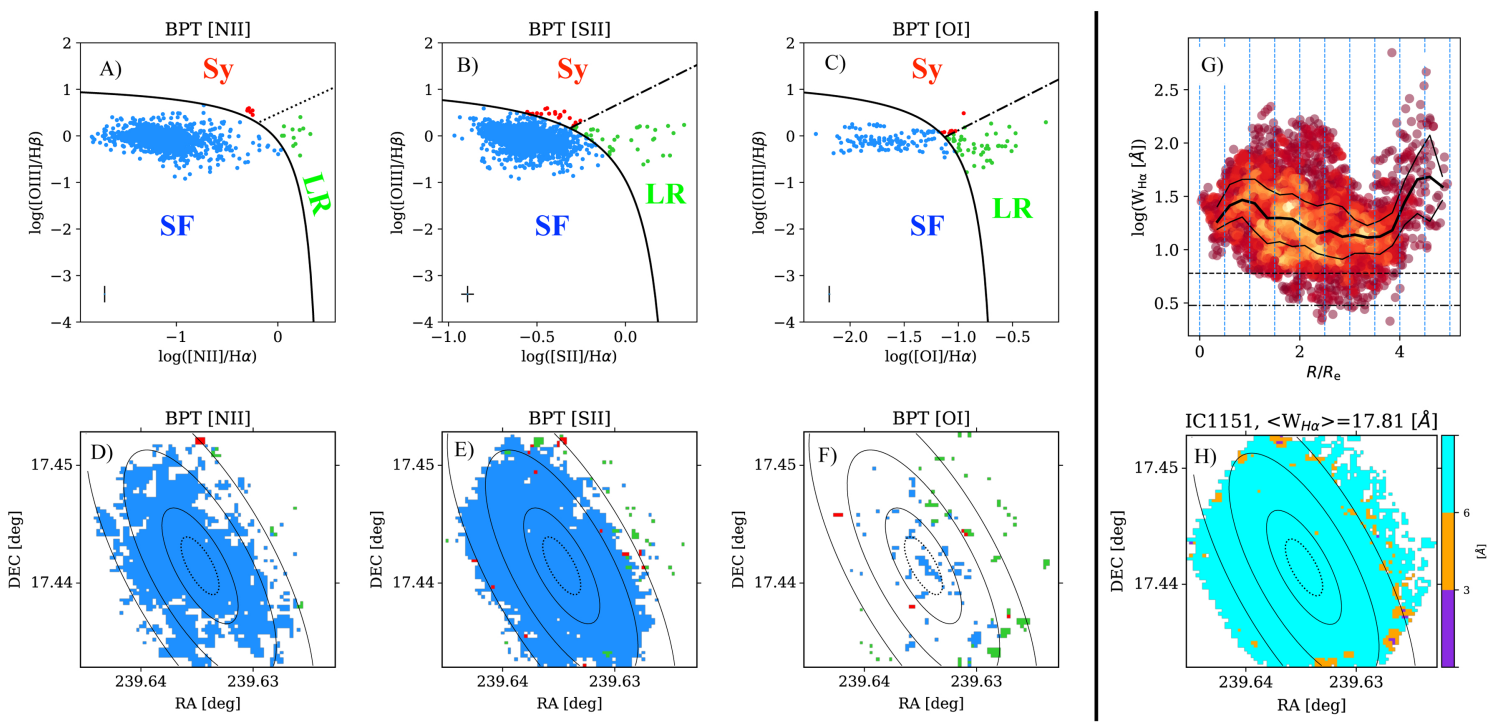

Fig. 2. Galaxy IC1151 as an example of the non-active class based on the BPT diagram and $W_{\mathrm{H} \alpha}$ criterion (see Sect. 3). Panels A, B, and $C$ : resolved [NII]-,[SII]- and [OI]-BPT diagnostic diagrams, where the solid curve is the empirical star-formation line of Kauffmann et al. (2003) and pixels below this line are dominated by star formation (SF; blue). The division (dash-dotted line) between the Seyfert (Sy; red) and LINER (LR; green) regions in panels $B$ and $C$ is adopted from Kewley et al. (2006), while the empirical Seyferts/LINER division (dotted line) in panel $A$ comes from Cid Fernandes et al. (2010). Panels D, E, and F: resolved [NII]-,[SII]-, and [OI]-BPT maps corresponding to the BPT diagram. Panels $G$ : resolved $W_{\mathrm{H} \alpha}$ radial profile from individual spaxels, where the dashed-dotted and dashed lines correspond to the thresholds of 3 and $6 \AA$, respectively. The blue dotted vertical lines define the bins of $0.5 R_{\mathrm{e}}$. The black thick line shows the median value of the $W_{\mathrm{H} \alpha}$, where the thin black lines indicate the median absolute deviation. Panel $H$ : resolved $W_{\mathrm{H} \alpha}$ map, where the colour bar indicates the thresholds of the spaxels: $W_{\mathrm{H} \alpha} \leq 3 \AA$ (violet), $3 \AA<W_{\mathrm{H} \alpha} \leq 6 \AA$ (orange) and $W_{\mathrm{H} \alpha}>6 \AA$ (cyan). The dotted line indicates the region of $0.5 R_{\mathrm{e}}$, where the continuous lines mark separation of 1,2 , and $3 R_{\mathrm{e}}$. The median value of the $W_{\mathrm{H} \alpha}$ for the full maps is given as label in the panel. To classify this galaxy as non-active, the central pixels that are below $0.5 R_{\mathrm{e}}$ should not populate the Seyfert region of the BPT diagrams (at least two from the three BPT diagrams), but their $W_{\mathrm{H} \alpha}$ values can vary, depending on the quenching stage of the galaxy.

intermediate-ionisation EL regions ( $3 \AA<W_{\mathrm{H} \alpha} \leq 6 \AA$ ), and lowionisation emission-line regions $\left(W_{\mathrm{H} \alpha} \leq 3 \AA\right.$ ) with no clear morphological pattern on the $W_{\mathrm{H} \alpha}$ map. The median value of $W_{\mathrm{H} \alpha}$ of the galaxy is usually between 3 and $6 \AA$. A low-ionisation EL region in the centre of the galaxies is possible, but it should be more extended than $0.5 R_{\mathrm{e}}$ (to be distinguishable from the centrally quiescent class) or randomly distributed within the disc. This mixed class can be represented by both active and nonactive galaxies.

(v) Nearly retired galaxies. The fifth row of panels (K, L and $\mathrm{M}$ ) of Fig. 1 exemplifies the nearly retired class galaxies (UGC10905, NGC 2554 and NGC 6081), where the median value of the $W_{\mathrm{H} \alpha}$ map is equal to or below $3 \AA$. Similar to the mixed class, nearly retired galaxies have no clear pattern, but these galaxies are mostly dominated by low-ionisation EL regions (i.e. above $90 \%$ of the spaxels usually have $W_{\mathrm{H} \alpha}$ values equal to or below $3 \AA$ ) and little star-formation within $2 R_{\mathrm{e}}$ of the galaxy (usually less than $3 \%$ of the spaxels have $W_{\mathrm{H} \alpha}>6 \AA$ ). This class cannot be mistaken with the centrally quiescent class in cases where there are low-ionisation ELs in the central regions because members of this class do not have a well-defined starforming disc beyond $0.5 R_{\mathrm{e}}$ as in the centrally quiescent class. The nearly retired class can be represented by both active and non-active galaxies.

(vi) Fully retired galaxies. Panel N of Fig. 1 represents the fully retired class of galaxies (NGC 5485), where the median value of $W_{\mathrm{H} \alpha} \leq 3 \AA$. The galaxy is fully dominated by these lowionisation EL regions within $2 R_{\mathrm{e}}$ of the galaxy. Similar to the centrally quiescent class, the fully retired class is entirely made up of non-active galaxies because the $W_{\mathrm{H} \alpha}$ value of the central pixels is always equal to or below $3 \AA$ even in cases where the line ratios populate the Seyfert region of the BPT diagrams. This class is somewhat similar to the eLIER galaxies in the [SII]-BPT classification of Belfiore et al. (2016).

\subsection{Nuclear activity criteria}

From the BPT diagrams in panels A-C of Figs. 2-4, we define whether the galaxy is active or non-active for each QS (i.e. this is the second dimension of the EL classification QuestNA). If the line ratios of the central spaxels that are below $0.5 R_{\mathrm{e}}$ populate the Seyfert region of the BPT diagrams (at least two from the three BPT diagrams), then the galaxy is defined as an AGNhost candidate (three is the minimum number of central pixels that define the nuclear activity of the galaxies based on the point spread function of CALIFA maps).

If the central pixels $\left(<0.5 R_{\mathrm{e}}\right)$ have values of $W_{\mathrm{H} \alpha}$ above $3 \AA$, the nuclear activity classification is reliable, and the galaxy is considered active; otherwise it is labelled non-active (galaxy is also classified as non-active if the line ratios do not populate the Seyfert regions). Once the galaxy is classified as active, we further define whether it hosts a weak AGN, where the $W_{\mathrm{H} \alpha}$ values of the central pixels range between 3 and $6 \AA$, or a strong AGN, where the $W_{\mathrm{H} \alpha}$ values of the central pixels go above $6 \AA$. The corresponding resolved maps of the BPT diagrams in panels D-F help us to locate the Seyfert spaxels, and decide whether or not they belong to the centre of the galaxy. Panel $\mathrm{G}$ and $\mathrm{H}$ show the resolved radial profile and the map of $W_{\mathrm{H} \alpha}$, respectively, 
Weak AGN galaxy: BPT diagram $+\mathrm{W}_{\mathrm{H} \alpha}$ criterion
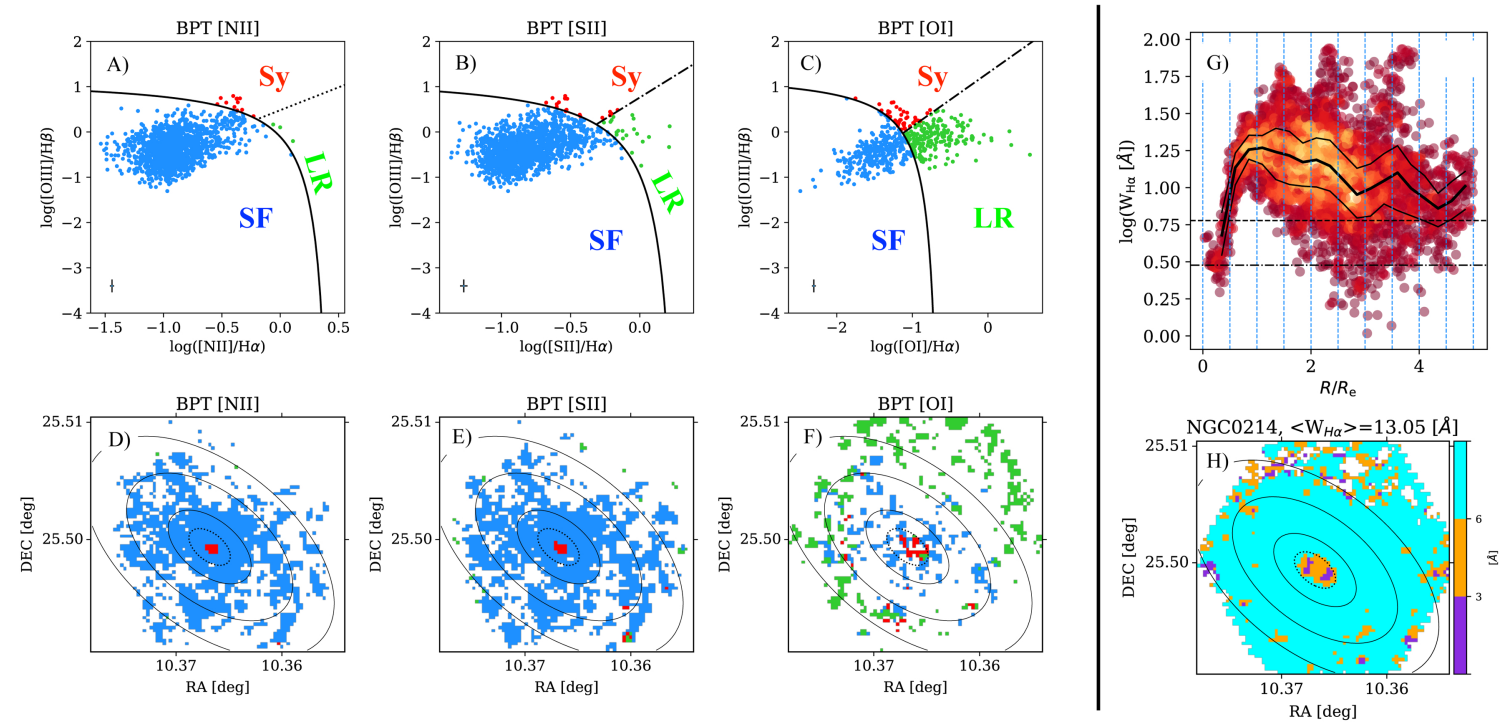

Fig. 3. Galaxy NGC 0214 as an example of a weak AGN class based on the BPT diagram and $W_{\mathrm{H} \alpha}$ criterion (see Sect. 3). Description as per Fig. 2 . To classify this galaxy as wAGN, the central pixels that are below $0.5 R_{\mathrm{e}}$ should have values $3 \AA<W_{\mathrm{H} \alpha} \leq 6 \AA$, and they should populate the Seyfert (Sy) region of the BPT diagrams (at least two from the three BPTs).

\section{Strong AGN galaxy: BPT diagram $+\mathrm{W}_{\mathrm{H} \alpha}$ criterion}
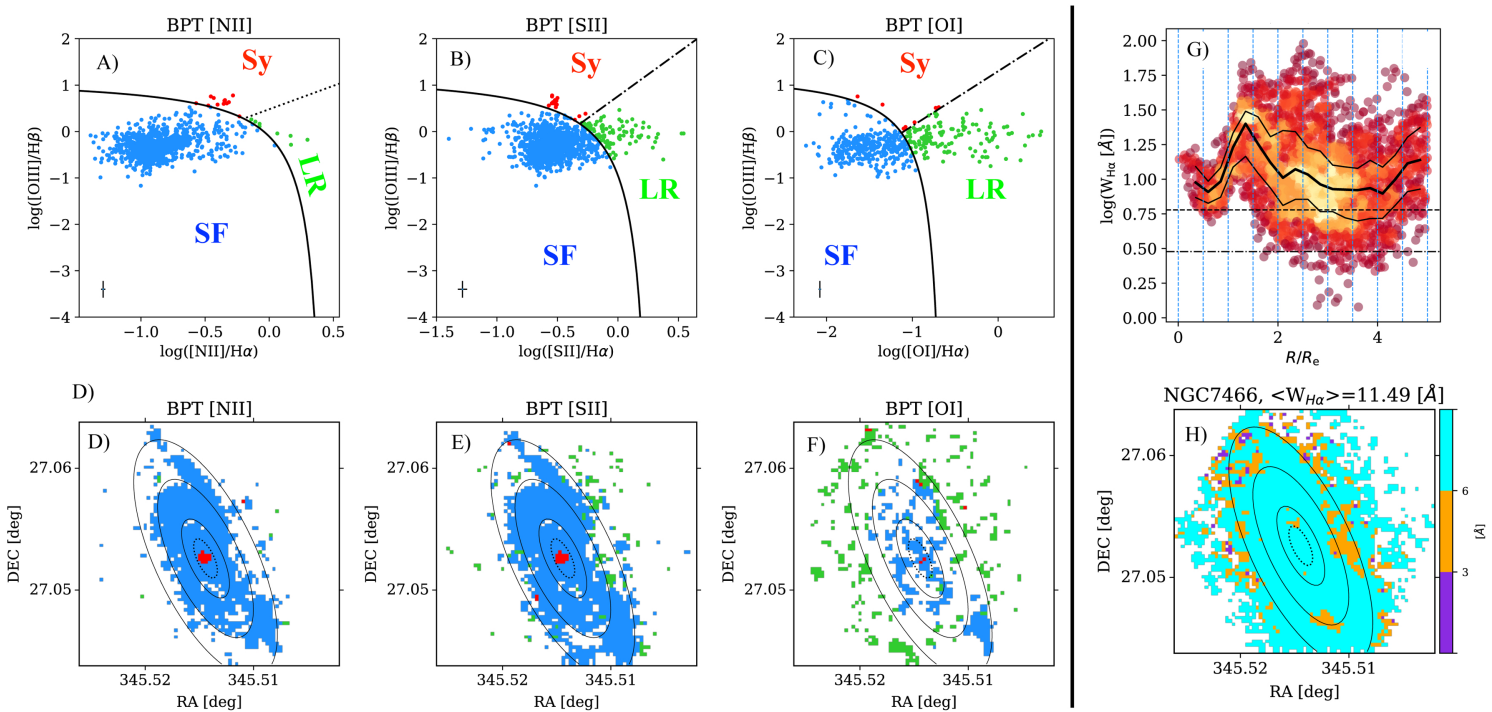

Fig. 4. Galaxy NGC 7566 as an example of a strong AGN class based on the BPT diagram and $W_{\mathrm{H} \alpha}$ criterion (see Sect. 3). Description as per Fig. 2. To classify this galaxy as sAGN, the central pixels that are below $0.5 R_{\mathrm{e}}$ should have $W_{\mathrm{H} \alpha}$ values above $6 \AA$ and they should also populate the Seyfert (Sy) region of the BPT diagrams (at least two from the three BPTs).

which are needed for applying the thresholds of the nuclear activity classification (i.e. 3 and $6 \AA$ of the $W_{\mathrm{H} \alpha}$ ). All resolved maps and BPT diagrams for the rest of the sample are shown in Appendix A.

\subsection{Uncertainties on the EL classification}

Using the scheme, we perform a by-eye classification of our galaxy sample, finding 96 star-forming, 12 quiescent-nuclearring, 23 centrally quiescent, 37 mixed, 34 nearly retired, and 36 fully retired galaxies (from which 215 non-active, 8 wAGN, and 15 sAGN). Despite the detailed analysis, there are marginal cases in our classification where a galaxy could be slightly below or above certain thresholds of the median $W_{\mathrm{H} \alpha}$ and/or its radial profile. Therefore, we attribute a quality index to our classified galaxies: a flag value of 1 indicates an unambiguous QS classification; flag=2 indicates uncertain QS classification due to complex $W_{\mathrm{H} \alpha}$ morphology; and flag $=3$ indicates uncertain $\mathrm{QS}$ classification due to bad data. Approximately $10 \%$ of the galaxies have uncertain QS classification (flag $=2$ or 3 ). On the other hand, the quality index, related to the nuclear activity classification includes sure $(S)$ and unsure $(U)$ cases, depending on whether the nuclear activity is confirmed by three or two of the BPT diagrams, respectively (nine galaxies have label=U, which is $\sim 4 \%$ of the total of 238 galaxies 
and $\sim 40 \%$ of the total active galaxies). The QS and NA classification labels, with the corresponding flags for all 238 CALIFA galaxies, are given in Table B.1.

For the AGN (weak and strong) classes, it is possible that the ionisation in the centre of the galaxy, shown in the Seyfert region of the BPT diagrams, is caused by star-formation-driven shocks rather than the AGN itself (e.g. López-Cobá et al. 2019). In this case, the galaxies typically show a bi-conical outflow from the centre of the galaxy to the outskirts, confirmed by the velocityto-velocity dispersion ratio of the galaxy in the central regions. López-Cobá et al. (2019) examined 667 CALIFA galaxies from DR3 (Sánchez et al. 2016a) in which our sample is included and defined 17 galaxies with star-formation-driven outflows. We compare our sample with theirs, and we only exclude the galaxy NGC 0681 from our list of the wAGN candidates due to the presence of outflow.

We also compare our AGN results with the list of bona fide AGN host galaxies, defined by Lacerda et al. (2020) from the extended sample of CALIFA survey (Sánchez et al. 2016a, Galbany et al. 2018). There is an overlap of 10 galaxies (eight galaxies in the sAGN class: UGC03995, MCG-0202-030, UGC00987, NGC 2410, IC2247, NGC 2639, IC0540, NGC 3160 and two galaxies in the wAGN class: NGC 2554 and NGC 6762) from the total 23 AGN (15 sAGN and 8 wAGN) host galaxies in our sample. We classify more galaxies as having AGN because of the more relaxed criteria we adopt in our study compared to Lacerda et al. (2020). Specifically, we require the Seyfert region of the BPT diagrams to be populated at least for two of the three diagrams but Lacerda et al. (2020) require population in all three diagrams. The goals of these two studies are different: Lacerda et al. (2020) focus on the bona fide cases of AGN hosts and exclude some boarder cases, while we aim to record any possibility of AGN activity in the galaxy. For example, NGC 1167, which has a strong AGN in the centre (e.g. Struve et al. 2010), is included in our AGN sample but would be excluded by the stricter requirements of Lacerda et al. (2020). Outflows and shocks are not included in the current 2D EL classification since it is out of the scope of the current study. On the other hand, it might be possible to add their categorisation to the nuclear activity criteria and expand the EL classes.

Finally, we might expect to find more EL classes or subclasses with improved spectral and spatial resolution data, and a higher number of sample statistics in comparison to the current sample (e.g. the AMUSING ++ survey, López-Cobá et al. 2020).

\section{Characteristic $W_{\mathrm{H} \alpha}$ radial profiles of the EL galaxies}

To classify the galaxies in their corresponding EL classes, we use set of figures and diagrams for each galaxy as discussed in Sect. 3. One important element is the $W_{\mathrm{H} \alpha}$ radial profile, which provides a detailed information about the distribution of the retired and the star-forming regions of the galaxies.

In Fig. 5, we show the characteristic (stacked) $W_{\mathrm{H} \alpha}$ radial profiles of all galaxies in each EL class. The profiles are obtained through a radial map positioned in the centre of the galaxy and projected on the plane of the galaxy, assuming the position angle and inclination reported in Table B.1 of K17. Similarly to Fig. 1, the rows of Fig. 5 correspond to the QSs of the galaxies, and the columns correspond to their nuclear activity groups. The nuclear activity leaves a clear trace within roughly $0.2 R_{\mathrm{e}}$ of the profiles: $W_{\mathrm{H} \alpha}>3 \AA$ for wAGNs (middle column), $W_{\mathrm{H} \alpha}>6 \AA$ for sAGNs (right column), while this value is not fixed for the non-active galaxies (left column).

Further, we compare the median profiles of the galaxy in each QS for the three different nuclear activity groups in Fig. 6. The details of those profiles vary significantly between the classes (especially within $0.5 R_{\mathrm{e}}$ ), but the average value of $W_{\mathrm{H} \alpha}$ generally increases from the centre of the galaxy to the outskirts. This might indicate that the star formation quenching in the galaxies largely proceeds 'inside-out', which has been noticed for CALIFA galaxies elsewhere (e.g. González Delgado et al. 2016; Belfiore et al. 2017; Lin et al. 2017; Ellison et al. 2018; Sánchez et al. 2018). Nevertheless, patterns can be more complicated as shown by the mixed and retired classes.

The star-forming galaxy group shows the highest radial values of $W_{\mathrm{H} \alpha}$, well above the $6 \AA$ threshold (see also panels A, B, and $\mathrm{C}$ in Fig. 5), where the radial median of $W_{\mathrm{H} \alpha}$ is larger than $13 \AA$ at each radii). This is particularly clear for the non-active group in the left panel of Fig. 6, which contains the largest statistical sample of galaxies.

Considering the nuclear activity in particular, the median profile of the sAGN galaxies is between 3 and $6 \AA$, which is below that of the non-active galaxies across the radial extent of the profile. The wAGN profile is roughly similar to the nonactive profile for the star-forming QS, except within $0.5 R_{\mathrm{e}}$ where they differ by $\sim 10 \AA$. Nevertheless, for the other QSs the difference between the profiles from the three nuclear activity groups is less evident.

Similarly high values $\left(W_{\mathrm{H} \alpha} \sim 10 \AA\right)$ are reached by the median radial profiles of quiescent-nuclear-ring and centrally quiescent classes beyond one effective radius. Within $1 R_{\mathrm{e}}$, instead, both profiles show a dip below $W_{\mathrm{H} \alpha}=3 \AA$ that characterises quenched regions (see also panels $D$ and $G$ in Fig. 5). Nevertheless, the central radial bin (where $R=0.25 R_{\mathrm{e}}$ ) of the quiescent-nuclear-ring class shows values that are again above the threshold that defined star formation dominance $(6 \AA)$.

It is interesting to note the case of the mixed profile, which is constantly in the range $3 \AA<W_{\mathrm{H} \alpha} \leq 6 \AA$, except in the central bins where the radial median of $W_{\mathrm{H} \alpha}$ is below $3 \AA$ (see also panel F of Fig. 5). This might be an indication that for this class, which generally presents a complex star formation pattern, the quenching begins from the very centre. Further, in Fig. 6, the mixed-wAGN profile in the regions of $R>2 R_{\mathrm{e}}$ goes above the $W_{\mathrm{H} \alpha}=6 \AA$ demarcation line (see also Fig. 5, panel I). This profile is generated by a single galaxy and may not be representative of the mixed-wAGN category.

Nearly retired and fully retired galaxy groups show $W_{\mathrm{H} \alpha}$ almost constantly below the $3 \AA$ threshold that marks ionisation by post-AGB star dominance. Both profiles increase in values from the centre to the outskirts, but the nearly retired profile is always above the fully retired median profile. In addition, the nearly retired galaxy group profile reaches values of $W_{\mathrm{H} \alpha}>3 \AA$, where $R>3 R_{\mathrm{e}}$, which might indicate some weak star formation activity in this region. The profiles from the AGN-host galaxies in the nearly retired stage are approximately equivalent to their non-active counterparts.

\section{Global properties of the galaxies at different quenching stages}

To understand the typical properties of each EL class, we investigate some basic parameters of the studied stellar systems. The results show that the EL classes are characterised by specific 


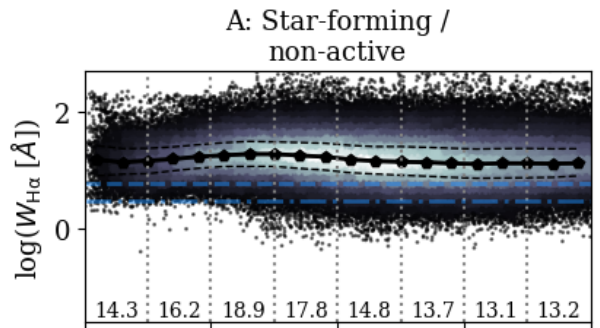

D: Quiescent-nuclear-ring /

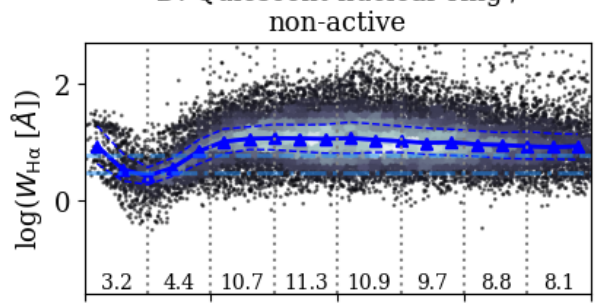

G: Centrally quiescent /

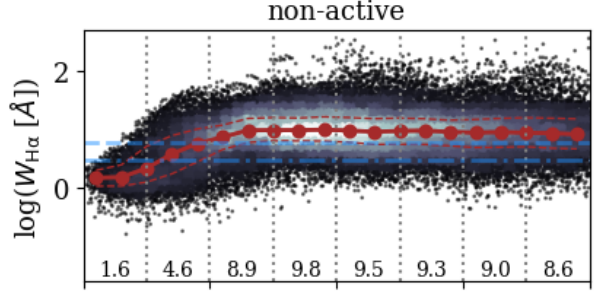

J: Mixed /

non-active

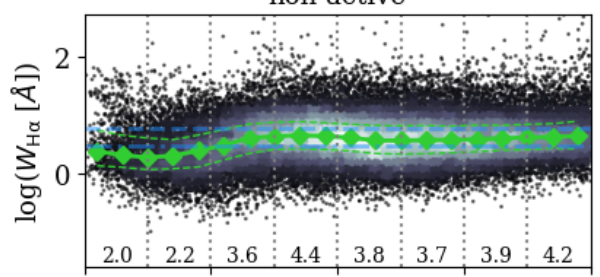

M: Nearly retired / non-active

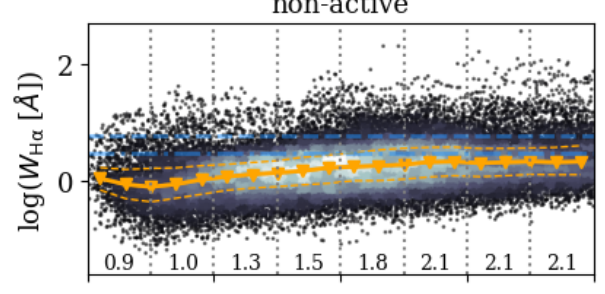

P: Fully retired / non-active

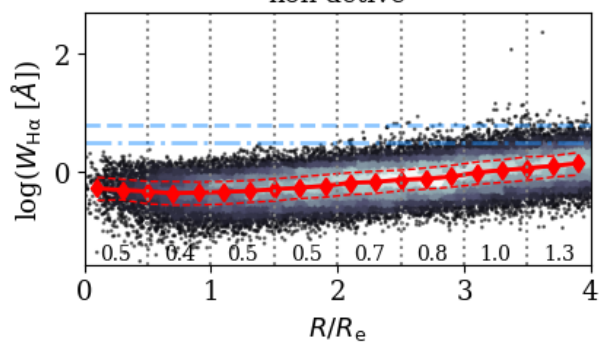

B: Star-forming / weak AGN

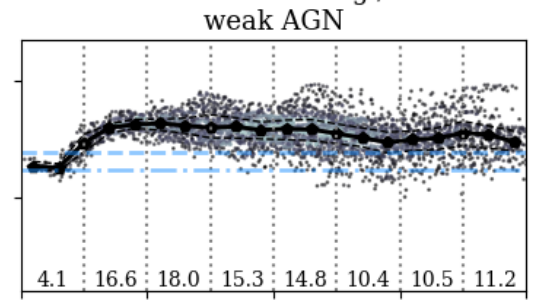

E: Quiescent-nuclear-ring / weak AGN

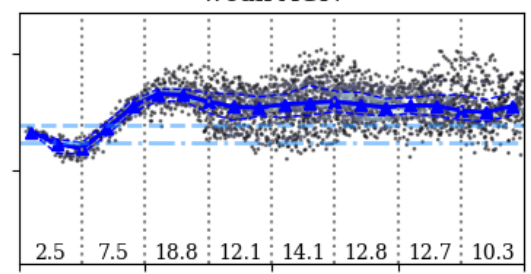

K: Mixed /

weak AGN

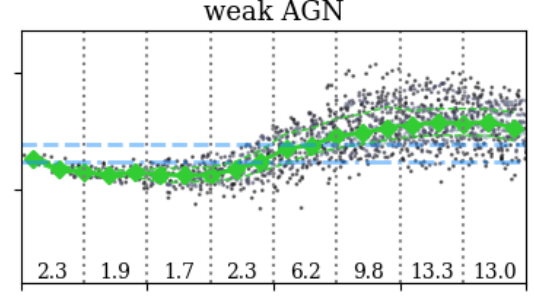

$\mathrm{N}$ : Nearly retired / weak AGN

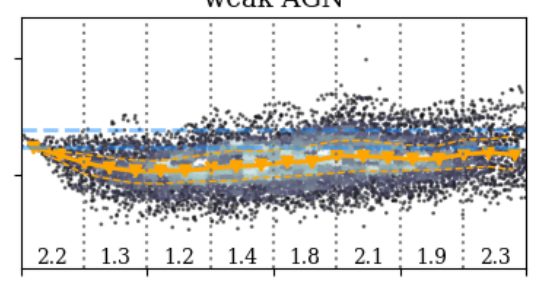

C: Star-forming / strong AGN

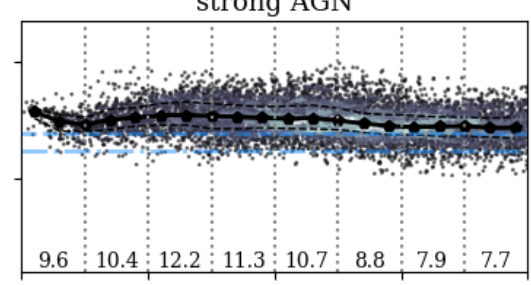

F: Quiescent-nuclear-ring / strong AGN

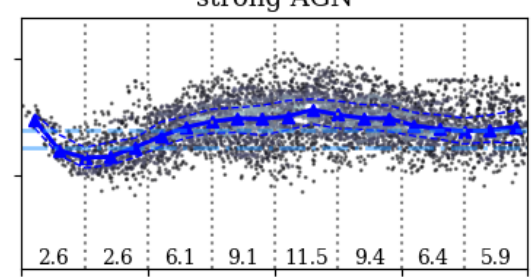

L: Mixed /

strong AGN

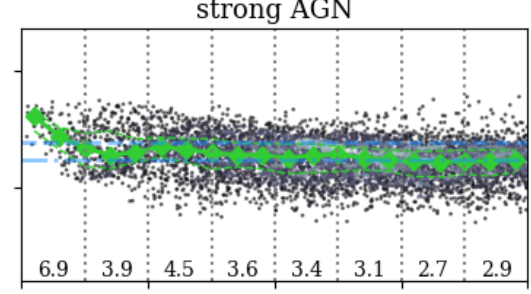

O: Nearly retired / strong AGN

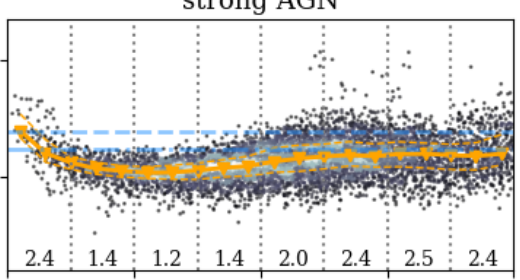

Fig. 5. Stacked radial profiles versus equivalent width of $\mathrm{H} \alpha\left(W_{\mathrm{H} \alpha}\right)$ for the galaxies in the different emission-line classes, normalised by galaxy effective radius $R_{\mathrm{e}}$ (Sect. 4). The coloured full lines show the median of the radial distributions, and the coloured dashed lines indicate the 25 th and 75th percentiles of the radial distributions. The blue dash-dotted and dashed horizontal lines display constant $W_{\mathrm{H} \alpha}=3 \AA$ and $W_{\mathrm{H} \alpha}=6 \AA$ values, respectively. The numbers at the bottom of each panel show the median $W_{\mathrm{H} \alpha}$ in a given radial bin of $0.5 R / R_{\mathrm{e}}$. Bins are separated by vertical, black, dashed lines. 

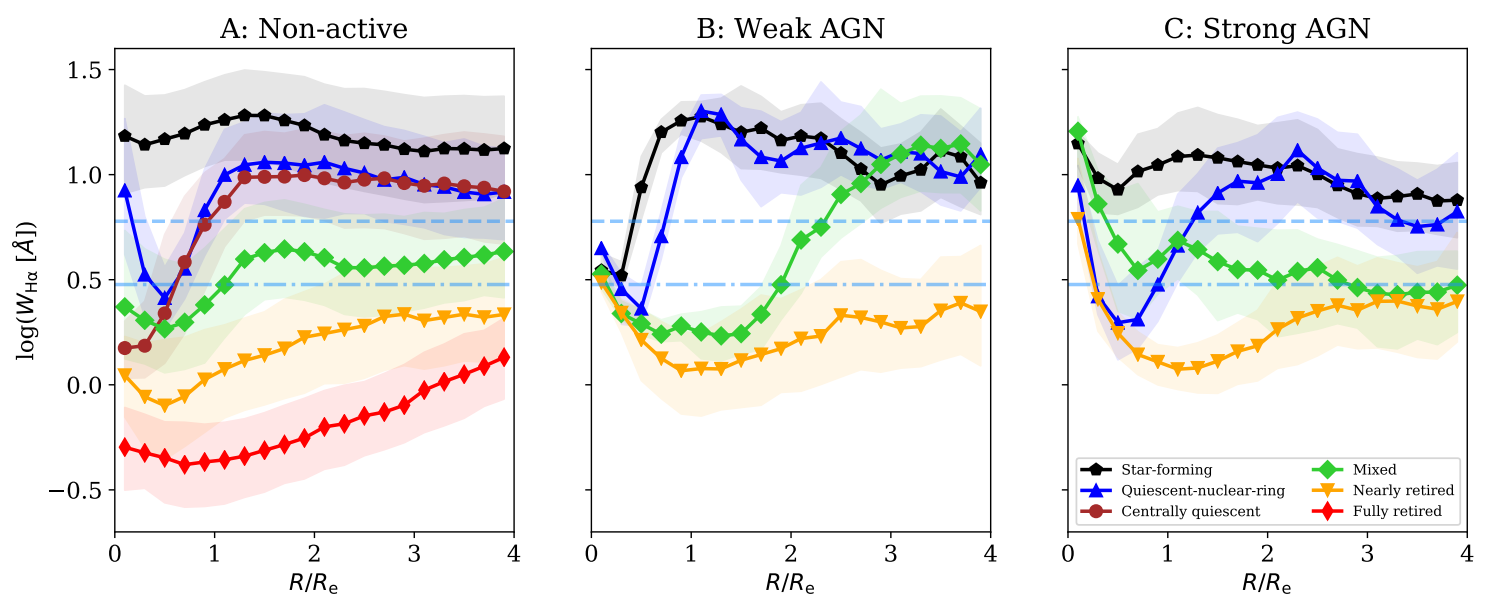

Fig. 6. Median radial profiles equivalent width of $\mathrm{H} \alpha\left(W_{\mathrm{H} \alpha}\right)$ for our sample of galaxies in each quenching stage for non-active (left), weak AGN (middle), and strong AGN (right) classes, normalised by galaxy effective radius $R_{\mathrm{e}}$ (Sect. 4). The shaded regions express the 25th and 75th percentiles of the radial distributions. The blue dash-dotted and dashed horizontal lines display $W_{\mathrm{H} \alpha}=3 \AA$ and $W_{\mathrm{H} \alpha}=6 \AA$ thresholds, respectively. The variation in the $W_{\mathrm{H} \alpha}$ profile suggests an 'inside-out' star-formation quenching scenario for our sample of galaxies.

properties of the galaxies, indicating that QuestNA is a valid classification method.

\subsection{Morphology}

The morphology of similar emission-line class galaxies has been broadly explored in the literature (e.g. Schawinski et al. 2007; Sánchez et al. 2018, 2019; Lacerda et al. 2020), and is an important parameter that gives direct information about the structural characteristics of the galaxies in the various categories. In Fig. 7 , we show the distributions of our EL classes through the Hubble sequence using the CALIFA classification of Walcher et al. (2014). In general, the morphology changes from late- to earlytype moving from the star-forming to the retired classes.

Examining the non-active galaxies (continuous lines is Fig. 7), the star-forming class are mainly late-type spirals (Sb-Sdm), while quiescent-nuclear-ring and centrally quiescent systems are represented by early-type spirals (Sab-Sbc). Similarly to the centrally quiescent and quiescent-nuclear-ring objects, the mixed class possesses early-type spiral morphologies $(\mathrm{Sa}-\mathrm{Sb})$, but it also includes a few cases of lenticulars ( $\mathrm{SO}$ S0a) and ellipticals (E4-E7). Furthermore, the nearly retired and fully retired classes of the non-active galaxies are mainly presented by lenticular and elliptical galaxies.

In total, the sAGN class (slashes in Fig. 7) is mostly represented by early-type spiral $(\mathrm{Sa}-\mathrm{Sbc})$ and lenticular $(\mathrm{S} 0-\mathrm{S} 0 \mathrm{a})$ galaxies. Similarly, the wAGN class (circles in Fig. 7) spans over the early-type spiral (Sa-Sbc) and lenticular (S0-SOa) systems, but also includes older galaxies such as the ellipticals (E4E7). The comparison between the distributions of the active and non-active galaxies shows a mismatch for the star-forming and nearly retired QSs. Panel B of Fig. 7 of the star-forming QS shows that the sAGNs are represented by early-type spiral galaxies (Sa-Sbc), which differs from the main peak of the non-active galaxies' distribution, located towards the late-type spirals. Furthermore, panel F of Fig. 7 of the nearly retired QS indicates that the wAGNs span over the early-type galaxies and early-type spirals, where the latter is an outlier region for the distribution of non-active galaxies, peaking at the lenticular galaxies.

We also make a structural comparison between the active and non-active group, taking into account the presence of a sure (B) or unsure $(\mathrm{AB})$ bar in each quenching group based on CALIFA bar classification of Walcher et al. (2014). Figure 8 shows that the highest number of barred galaxies belong to the quiescentnuclear-ring QS, followed by the centrally quiescent and mixed groups. The upper error-bar of the histograms represents the maximal number of bars in a group, which includes both bars (B) and unsure bar (AB) cases of the galaxies (see also Table B.1). The information from the morphological and bar classifications of Walcher et al. (2014) for our sample suggest that the secular evolutionary processes (which involve the internal evolutionary phenomena induced by dynamical features such as spiral arms and bars) are much more prominent in the quiescent-nuclearring, centrally quiescent, and mixed classes than in the remaining classes.

\subsection{Photometric, stellar, kinematic, and dynamical properties}

Besides the galaxy morphology, our classification correlates with a large set of galactic properties as shown in Fig. 9. This proves that knowledge of the QS of a galaxy is sufficient to access the average value of a variety of parameters. The bulgeto-disc ratios $(\mathrm{B} / \mathrm{D}$, panel $\mathrm{A})$; the stellar mass surface density $\left(\mu_{*}\right.$, panel $\left.\mathrm{D}\right)$; the stellar age $(\tau$, panel $\mathrm{F})$; the stellar metallicity $(Z$, panel $\mathrm{G})$; and the peak circular velocity $\left(V_{\mathrm{c}, \max }\right.$, panel $\left.\mathrm{J}\right)$ all increase moving from the star-forming to the fully retired groups for the non-active galaxies. Conversely, the SFR sharply decreases across the QSs (panel $\mathrm{H}$ ).

For other properties, the trends are less obvious. Regarding the total luminosity ( $L_{r}^{\text {tot }}$, panel B), the brightest galaxies, on average, are observed within the nearly retired class, and not within the fully retired ones, while galaxies in the star-forming class are the faintest objects. A similar trend is shown by total stellar and dynamical mass $\left(M_{*}\right.$, panel E; and $M_{\mathrm{dyn}}^{\text {tot }}$, panel $\mathrm{K}$; respectively), while the opposite behaviour is observed for angular momentum $\left(\lambda_{R_{\mathrm{e}}}\right.$, panel I). The size of the galaxies inferred from the median effective radius, $R_{\mathrm{e}}$, shows a complicated behaviour across the stages (panel $\mathrm{C}$ ). The largest-size galaxies are mainly quiescent-nuclear-ring galaxies $\left(R_{\mathrm{e}} \sim 5.35 \mathrm{kpc}\right)$ and nearly retired $\left(R_{\mathrm{e}} \sim 4.89 \mathrm{kpc}\right)$, followed by the centrally quiescent $\left(R_{\mathrm{e}} \sim 4.81 \mathrm{kpc}\right)$. Fully retired galaxies have the smallest $R_{\mathrm{e}} \sim 2.23 \mathrm{kpc}$ in comparison to the rest of the classes, which might indicate that most of the luminosity is generated by their large spheroidal components (considering their large B/D ratio). 


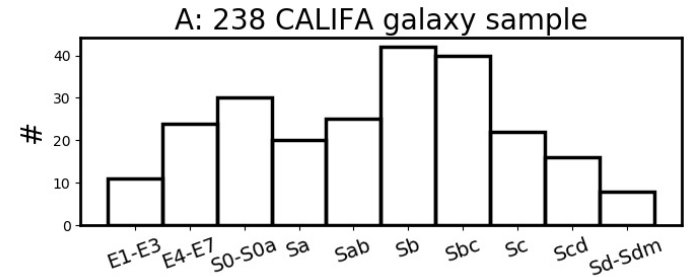

B: Star-forming

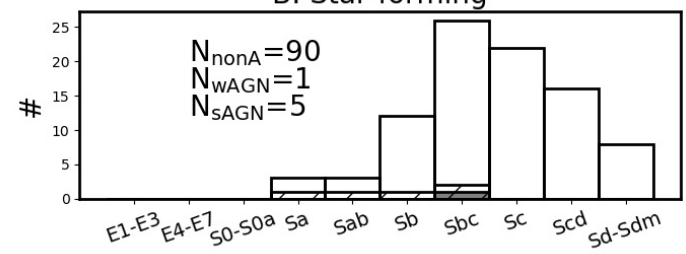

C: Quiescent-nuclear-ring

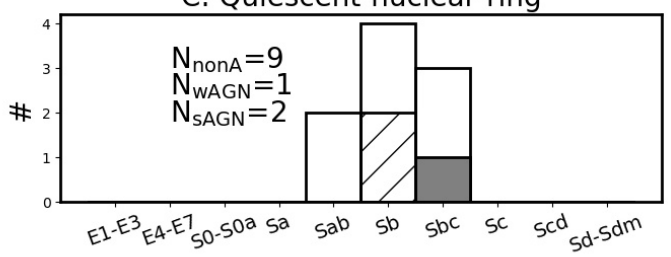

D: Centrally quiescent

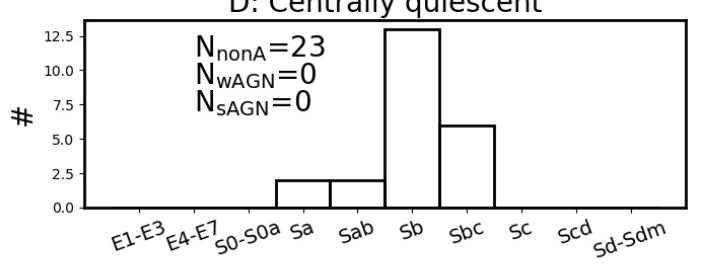

E: Mixed

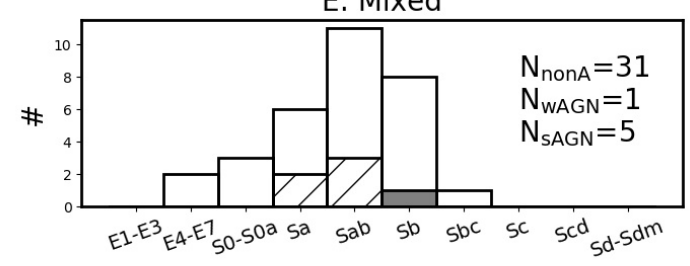

F: Nearly retired
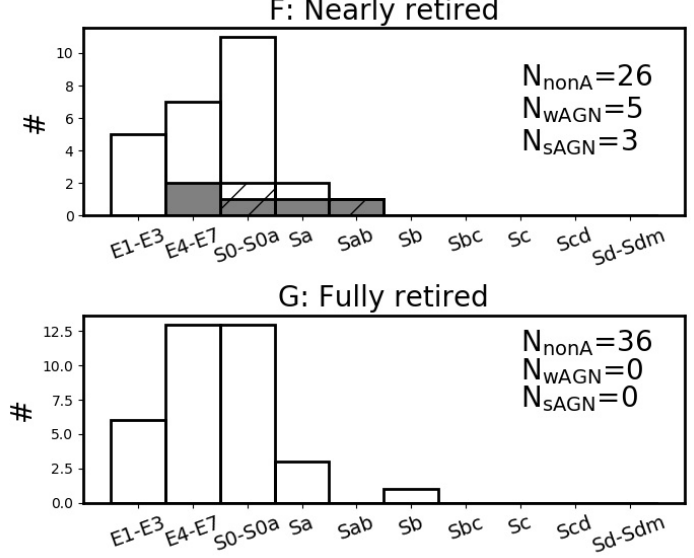

Fig. 7. Distributions of the active and non-active galaxies in each quenching stage through Hubble type (panels $B-G$ ). The blank bars outline the distribution of the non-active galaxies, while the grey-filled bars and the slashes correspond to the wAGN and sAGN distributions, respectively. Panel $A$ : distribution of the full sample. $N$ gives the number of the galaxies for each nuclear activity group (see Sect. 5.1).

The mass discrepancy factor $f_{\mathrm{d}}=1-\left(M_{*}^{\text {tot }} / M_{\mathrm{dyn}}^{\text {tot }}\right)$ (panel L) displays a rather flat behaviour. We find that $f_{\mathrm{d}}$ is largest for star-forming galaxies $\left(f_{\mathrm{d}}=80 \%\right)$ in comparison to the
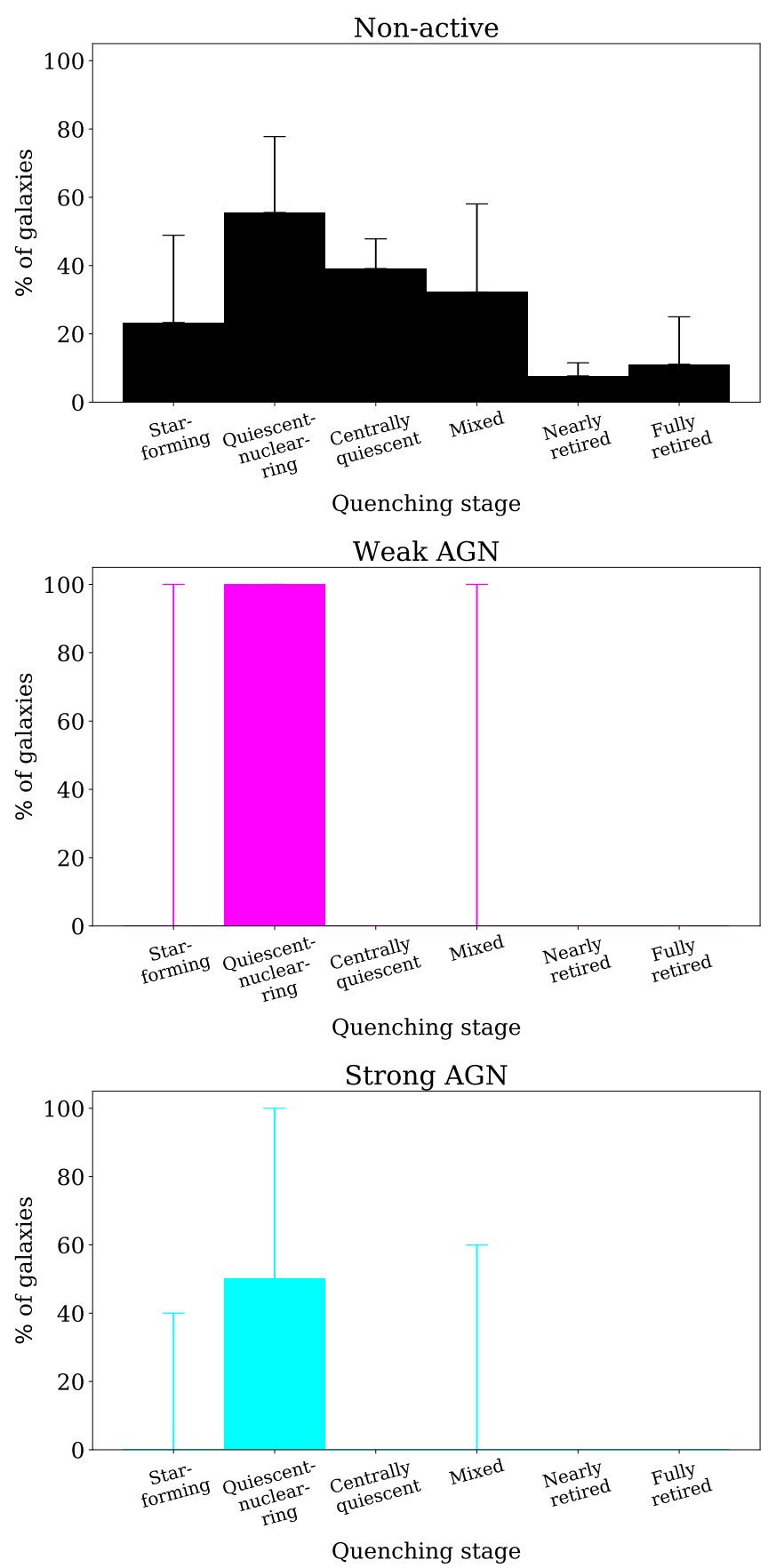

Fig. 8. Distributions of the barred galaxies through active and nonactive galaxies for each quenching stage of our sample. The upper errorbar for each stage indicates the fraction of galaxies with the unsure bar. Quiescent-nuclear-ring is represented by the highest number of barred galaxies, followed by centrally quiescent and mixed groups (see Sect. 5.1).

rest of the quenching classes, where $f_{\mathrm{d}}$ varies between 64 and $68 \%$. The large value of $f_{\mathrm{d}}$ through EL classes could be due to the non-universal nature of the galaxy IMF (e.g. Hoversten \& Glazebrook 2008; Cappellari et al. 2012; Lyubenova et al. 2016), radial variation of stellar mass-to-light ratio $\gamma_{*}$ (e.g. GarcíaBenito et al. 2019), or the presence of dark matter mass (e.g. Das et al. 2020). The variation of the mass discrepancy could also be explained with an uncounted gas mass in the galaxies, and this is expected to be significant for late-type systems (e.g. McGaugh et al. 2000). In forthcoming papers, we will infer the total gas 
mass fractions of our sample using a dust-to-gas calibration (see Barrera-Ballesteros et al. 2018) or CO data (from Bolatto et al. 2017; Colombo et al. 2020) and HI data (Kalinova et al., in prep.) to calculate the dark matter content for each galaxy. The median values of the galaxy properties from the box-and-whisker diagrams are listed in Table B.2.

The behaviour of the AGN-host galaxies at a given QS is also reported in Fig. 9. The sAGN galaxies within the star-forming class are the objects that show the most discrepant behaviour with respect to their non-active counterparts. Those galaxies are mostly located in the upper end of the distributions of all parameters, except for the SFR (panel H), where sAGN galaxies show global SFRs similar to the non-active objects in the same class. However, star-forming-sAGN appear between the objects of the star-forming class with the lowest $f_{\mathrm{d}}$. AGN-host galaxies that belong to the mixed and nearly retired categories seem to show low values of $R_{\mathrm{e}}$.

\section{Discussion}

\subsection{Imprints of galaxy evolution in the EL classes}

To understand whether our proposed categories are linked to galaxy evolution, we superimpose the EL classes on the SFR$M_{*}$ diagram (Fig. 10). Galaxies in the star-forming class almost fully populate the SFMS (Fig. 10, panel B). However, the AGNhost galaxies at this QS mostly show strong AGN activity and are located at the upper end of the SFMS, occupied by highmass galaxies. Quiescent-nuclear-ring and centrally quiescent galaxies appear to populate the green valley of star formation, which is generated by objects that are switching off their star formation in the central region (Fig. 10, panel C and D, respectively). Galaxies with an undefined distribution of the ionisation (mixed class) spread from the SFMS to the red sequence, but they appear mostly located in the green valley as well (Fig. 10, panel E). Strong AGN-host galaxies appear well distributed across the full high-mass region of the SFR- $M_{*}$ diagram. Nearly retired and fully retired galaxies are tightly constrained along the red sequence. In particular, nearly and fully retired galaxies dominate the high-mass and low-mass ends of the red sequence, respectively. It is interesting to note that the nearly retired galaxies are the most massive systems from our sample, which are also the most dispersion-dominated galaxies (i.e. they have the lowest $\lambda_{R_{\mathrm{e}}}$, panel I of Fig. 9) with the largest size stellar discs among the other classes (panels A and C of Fig. 9). These latter are old stellar population galaxies, but still have a little star-formation activity within the $2 R_{\mathrm{e}}$. This suggests that maybe the fully retired class are the progenitor of the nearly retired class, where star formation has been reignited in the galaxies by merger events or environmental processes in earlier epochs, or that the two classes might have followed independent evolutionary paths since the beginning of their formation (or a combination of both mechanisms). To understand more about the origin of the nearly- and fully retired galaxies, in our next article we will link the QS of the galaxies and their dynamical properties (Kalinova et al., in prep.).

Further, AGN galaxies at the nearly retired stage appear to be mostly in the 'weak' category. This might indicate that the AGN activity reduces with the evolution of the galaxies and the progression of the quenching, possibly due to the progressive diminution of the gas supply.

Panels B and C collect the position of all wAGN and sAGN galaxies independently of their quenching stage. The location of the AGN host galaxies in the transition region between the blue cloud and the red sequence has been reported in other works (e.g. Schawinski et al. 2014; Sánchez et al. 2018; Cano-Díaz et al. 2019; Lacerda et al. 2020). The wAGN galaxies are largely dispersed between the green valley and the red sequence, and mostly occupy either the low-mass or high-mass end of the SFR$M_{*}$ diagram. Further, they also show bi-modality in their properties (see also Heckman \& Best 2014).

A quicker way to investigate the properties of the galaxies in respect to their position on the SFR- $M_{*}$ diagram and connect to their stage of evolution (e.g. Ellison et al. 2018; Thorp et al. 2019; Bluck et al. 2020) is provided by Fig. 11, which summarises the distribution of $\triangle \mathrm{SFR}$, where $\triangle \mathrm{SFR} \equiv \log \mathrm{SFR}-\log$ SFMS. Here, $\triangle$ SFR is the logarithmic distance from the SFMS of Elbaz et al. (2007) using the method described in Bluck et al. (2016). In the figure, the normalised histogram represents local galaxies from SDSS DR7 with a given $\triangle$ SFR. In general, the SDSS histogram in grey highlights the fact that galaxies at $z \sim 0$ are mostly located in the red sequence, followed by the blue cloud, and the green valley. In the figure, the QSs of non-active galaxies are indicated with the median and the median absolute deviation of $\Delta$ SFR of the given subsamples. Quenching-stage galaxies appear well divided through $\Delta$ SFR, indicating how a given QS represents a relatively narrow range of certain physical parameters, validating our classification as a useful tool to trace galaxy evolution through the star formation and nuclear activity. Only the median absolute deviation bars of mixed and centrally quiescent classes overlap in the green valley region.

Furthermore, in Fig. 11 for a given QS, we explore how the $\triangle \mathrm{SFR}$ values of the active galaxies (grey star for sAGN and grey square for wAGN) are comparable with the $\triangle \mathrm{SFR}$ median value (coloured symbols) and median absolute deviation (colour bars) of the non-active systems. Overall, star-forming sAGN galaxies show the biggest mismatch with the distribution of their non-active counterparts; they are all shifted towards lower $\triangle$ SFR values (i.e. they all deviate from the SFMS) than the star-forming non-active objects. This suggests that the suppression of the starformation has already started in these galaxies and might be related to the presence of the AGN in their centres through feedback processes (e.g. Frigo et al. 2019; Kauffmann \& Haehnelt 2000).

\subsection{Inside-out quenching and the role of AGNs}

Through our EL classification, we observe that the galaxies on their way to quenching always begin to switch off their star formation from the centre (see Fig. 6). The quiescent-nuclearring galaxies are an exception (because they have some instance of star formation below $0.2 R_{\mathrm{e}}$ ) but also a rare example, and they are mostly quenched within $0.3-0.5 R_{\mathrm{e}}$. In other words, we rarely observe a galaxy that is largely star forming in the centre and fully quenched in the outskirts. Usually, this is due to the presence of a close companion or interaction between galaxies (which have been purposely excluded from the sample analysed here). Outside-in star-formation quenching may also be induced by ram-pressure stripping in galaxy cluster environments (e.g. Bekki 2009; Vulcani et al. 2020).

Our results are consistent with an 'inside-out' quenching scenario (e.g. González Delgado et al. 2016; Catalán-Torrecilla et al. 2017; Belfiore et al. 2018; Sánchez et al. 2018; Lin et al. 2019; Lacerda et al. 2020), which predicts that the starformation quenching starts from the centre of the galaxies due to the presence and the continuous growth of the bulge (e.g. Martig et al. 2009). Galaxies with a larger central mass concentration are expected to host more massive black holes (BHs) from the $M_{\text {bulge }}-M_{B H}$ relation (e.g. Marconi \& Hunt 2003; Häring \& Rix 2004; McConnell \& Ma 2013), and experience more significant energetic feedback. Thus, the growth of the 


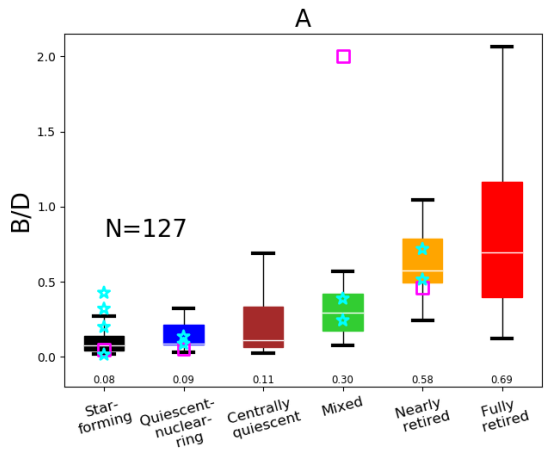

Emission-line class

D

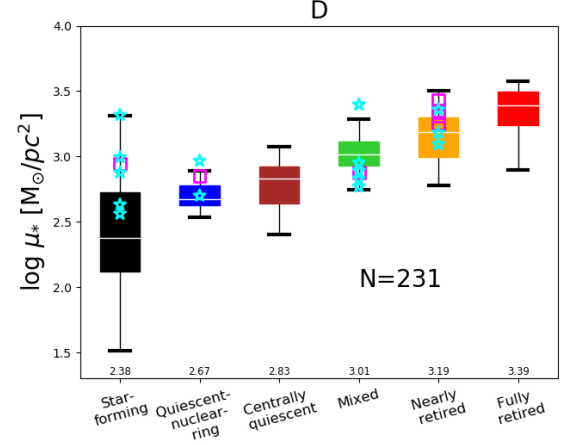

Emission-line class

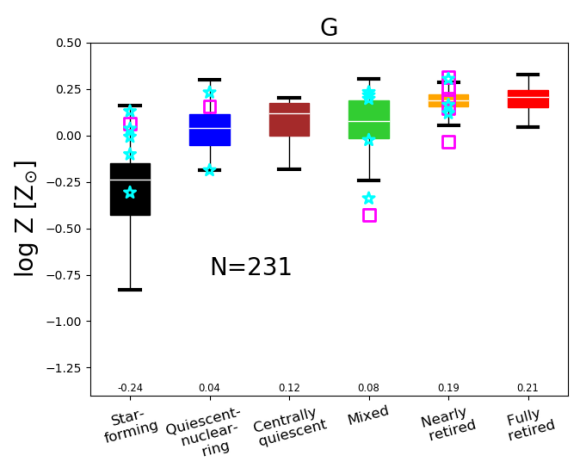

Emission-line class

।

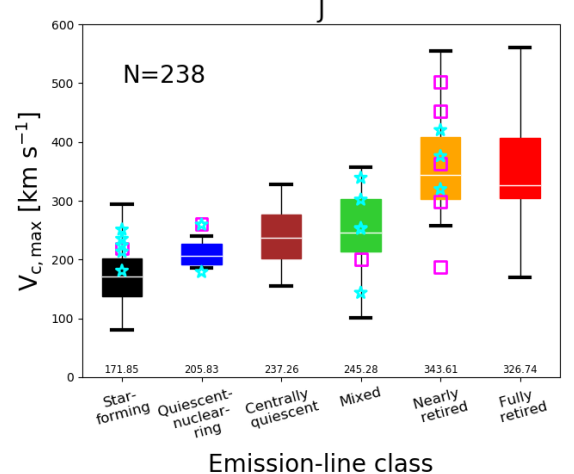

B

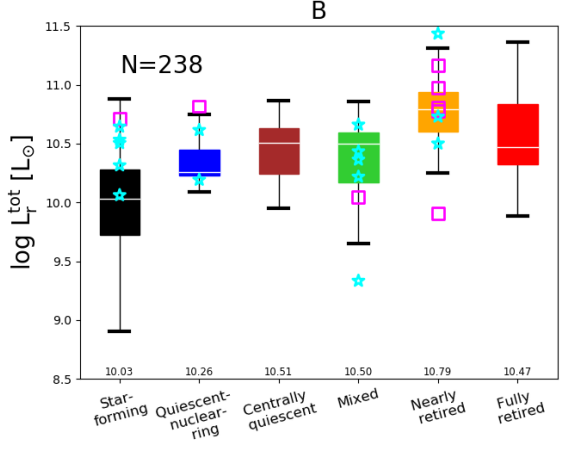

Emission-line class

E

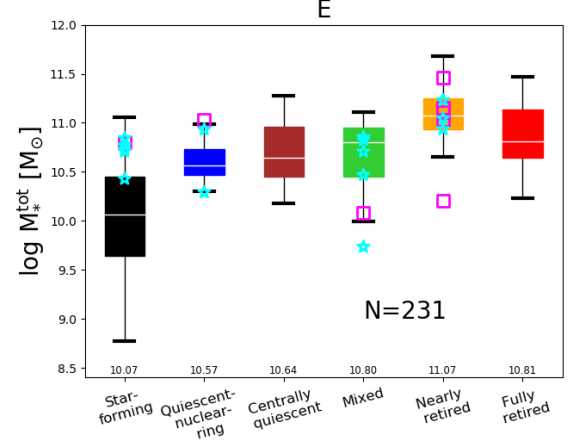

Emission-line class

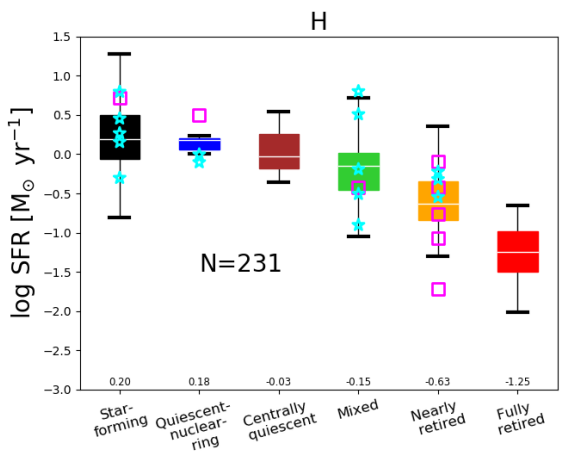

Emission-line class

$\mathrm{K}$

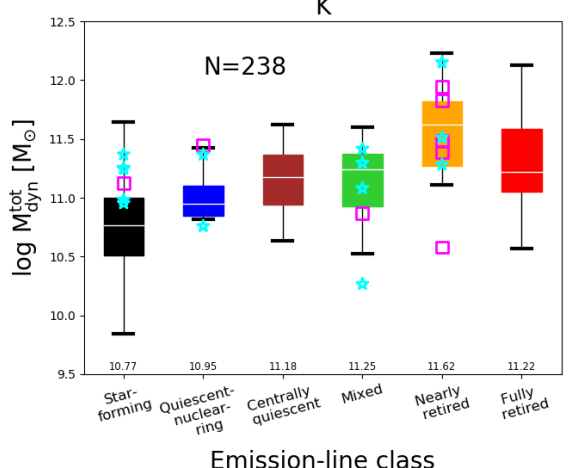

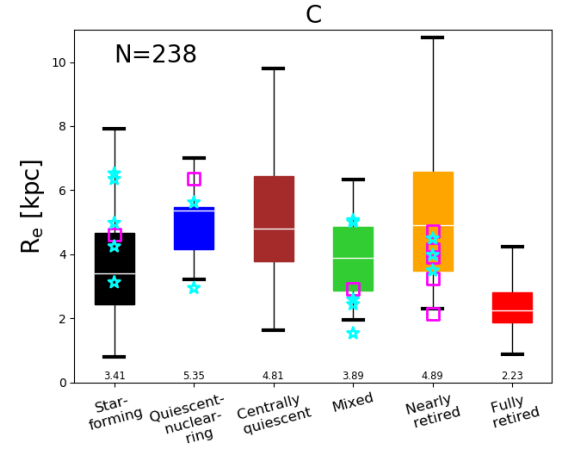

Emission-line class

$\mathrm{F}$

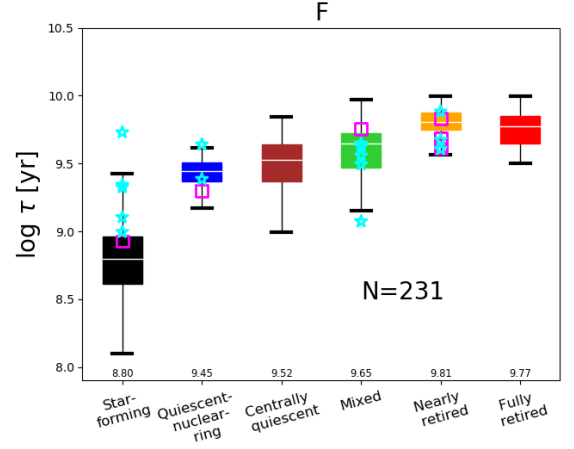

Emission-line class

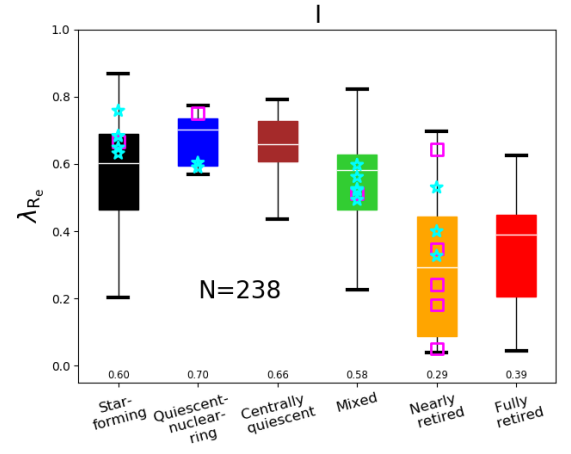

Emission-line class

L

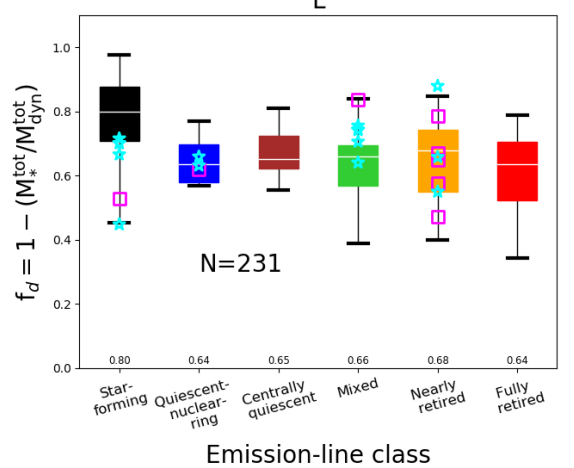

Fig. 9. Box-and-whisker diagrams of basic galaxy properties through the EL classification (see Sect. 5). The colour diagrams represent the properties of the non-active galaxies as follows: black - star-forming; dark blue - quiescent-nuclear-ring; brown - centrally quiescent; green - mixed; orange - nearly retired; and red - fully retired. The active galaxies are over-plotted with empty symbols on the diagrams for a given quenching stage (cyan star for sAGN and magenta square for wAGN). The boxes represent the data between the lower and the upper quartiles of the distribution with median values (Tukey 1977), given by the white lines and the numbers. The extent of the data outside the quartiles (whiskers) is indicated by the vertical black lines. The label ' $N$ ' in each panel gives the total number of the galaxies participating in the statistics. These diagrams represent distributions of various galactic properties in the different emission-line classes: bulge-over-disc flux ratio (B/D, panel $A)$; total luminosity in $r$-band $\left(L_{\mathrm{r}}^{\text {tot }}\right.$, panel $\left.B\right)$; effective radius $\left(R_{\mathrm{e}}\right.$, panel $\left.C\right)$; stellar mass surface density $\left(\mu_{*}\right.$, panel $\left.D\right)$; stellar mass $\left(M_{*}\right.$, panel $\left.E\right)$; stellar age $(\tau$, panel $F)$; stellar metallicity $(Z$, panel $G)$; star formation rate (SFR, panel $H)$; specific angular momentum $\left(\lambda_{R_{\mathrm{e}}}\right.$, panel $\left.I\right)$; peak circular velocity $\left(V_{\mathrm{c}, \max }\right.$, panel $\left.J\right)$; total dynamical mass $\left(M_{\mathrm{dyn}}^{\mathrm{tot}}\right.$, panel $\left.K\right)$; and mass discrepancy factor $\left(f_{\mathrm{d}}\right.$, panel $\left.L\right)$. The SF-sAGN galaxies show the most discrepant behaviour with respect to their non-active counterparts. The median values of the galaxy properties from the box-and-whisker diagrams are given in Table B.2. See text in Sects. 2 and 5 for further details. 

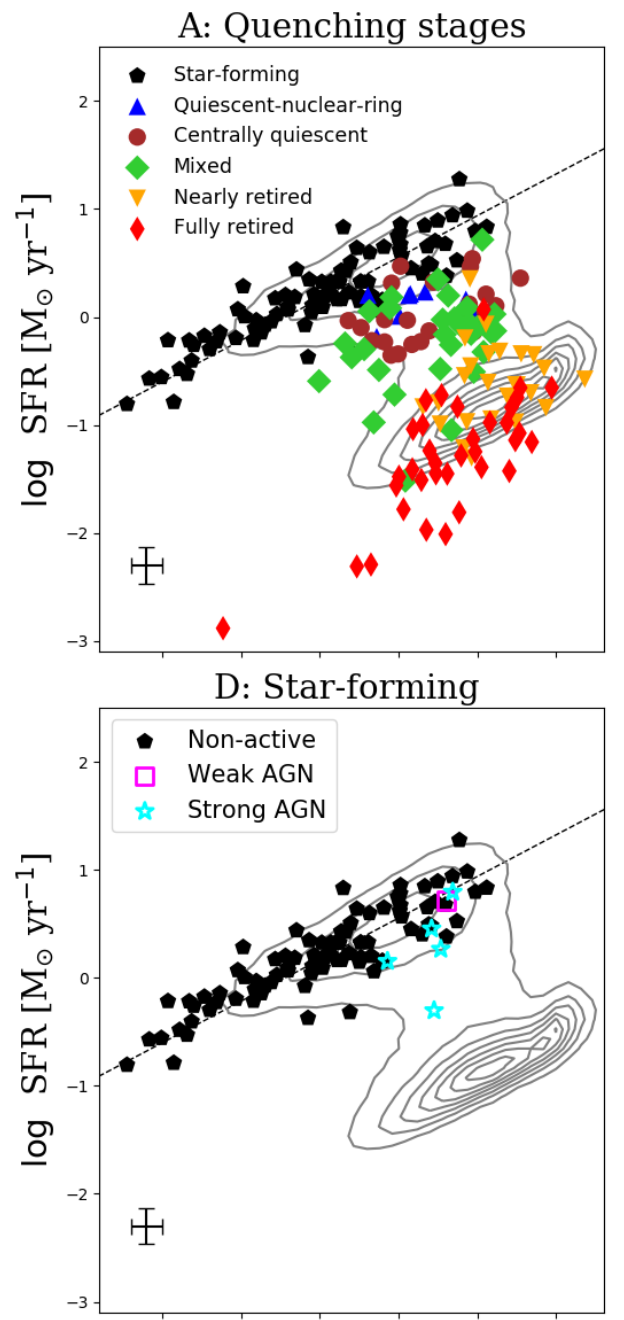

H: Mixed

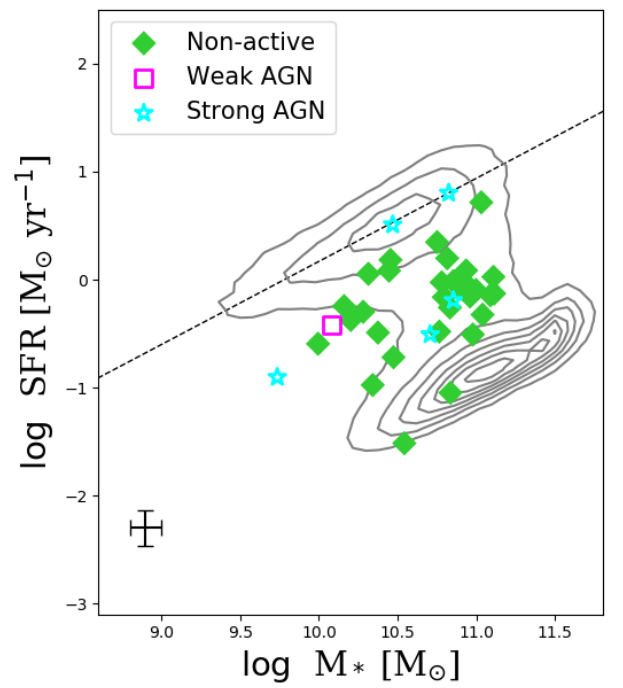

B: Weak AGN

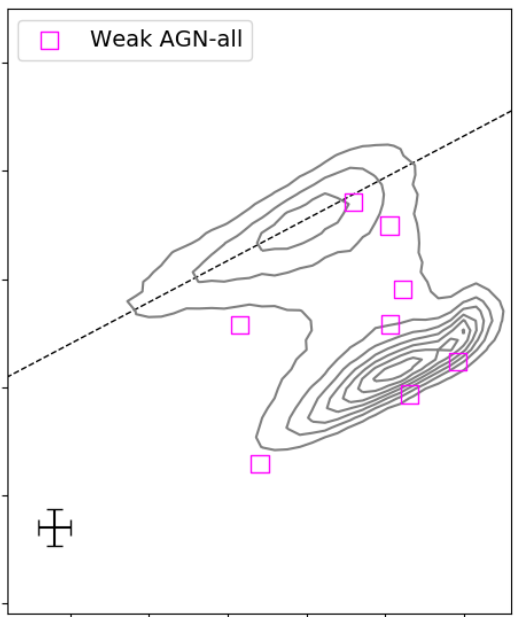

F: Quiescent-nuclear-ring

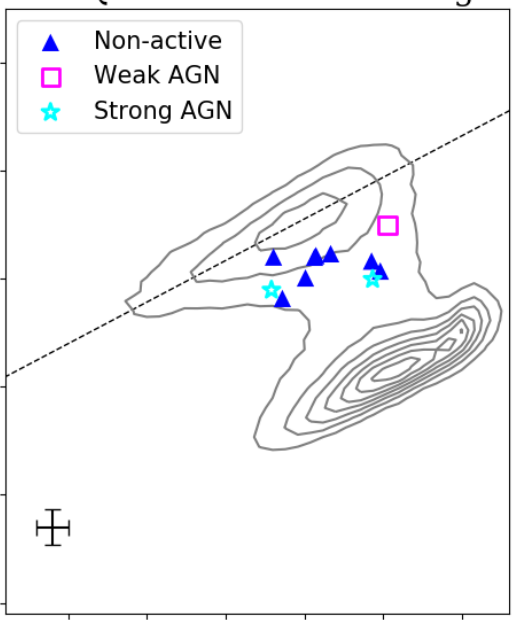

I: Nearly retired

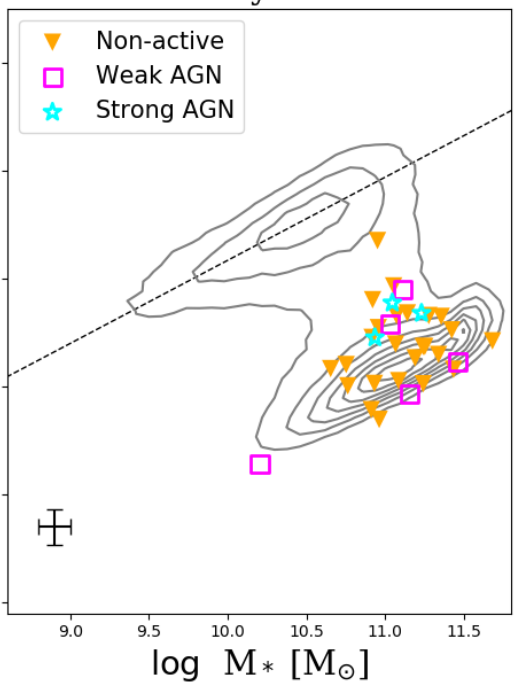

C: Strong AGN

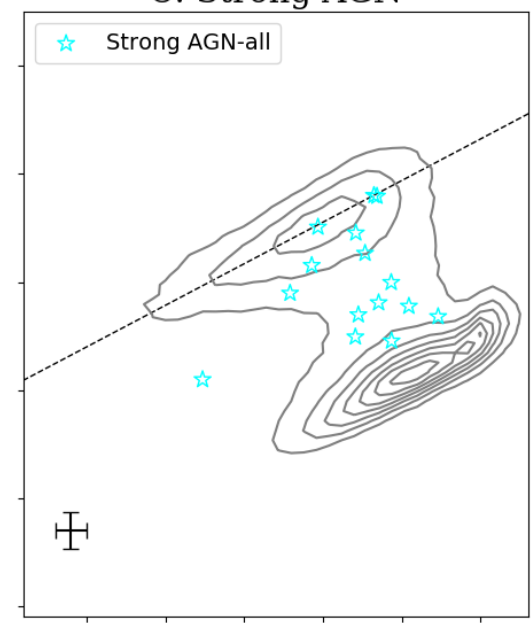

G: Centrally quiescent

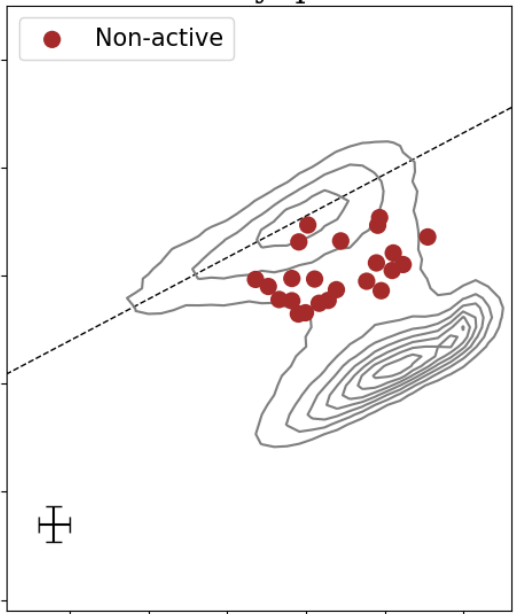

$\mathrm{J}$ : Fully retired

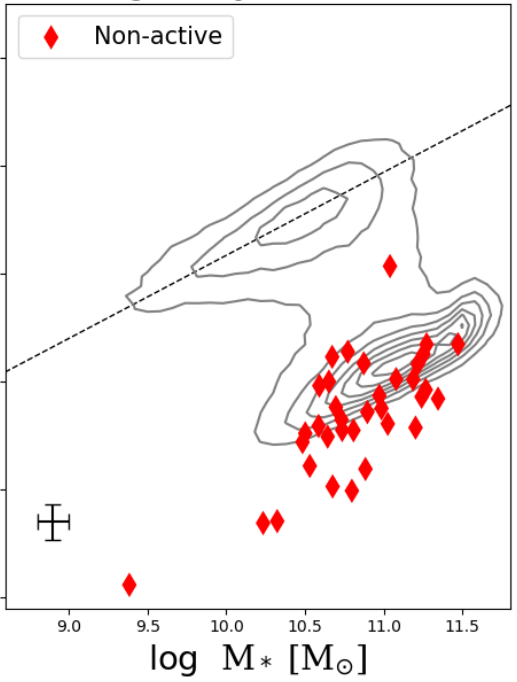

Fig. 10. SFR- $M_{*}$ for our sample of CALIFA galaxies, considering their quenching stages and nuclear activities (see Sect. 6.1). All active and non-active galaxies through quenching stage are presented in panel A. Panels B and C: galaxies that host a weak or strong AGN (respectively), independently of their quenching stage. Panels $D-J$ : galaxies in a given quenching stage, where the filled symbols represent the non-active galaxies, while the empty symbols correspond to the active galaxies: wAGN (magenta squares) or sAGN (cyan stars). The dashed line indicates the SFMS using the fit of Elbaz et al. (2007). The grey contours represent the SDSS DR7 population for reference from the MPA-JHU catalogue (Kauffmann et al. 2003; Brinchmann et al. 2004; Salim et al. 2007). The error bars in the panels represent the typical uncertainties on the stellar masses and the SFRs, adopted from Cid Fernandes et al. (2014) and González Delgado et al. (2017), respectively. The degree of star-formation quenching increases along the present EL-pattern sequence from star-forming to fully retired. For a given QS, the active galaxies occupy the same domain on the SFR- $M_{*}$ diagram as the non-active ones. 
V. Kalinova et al.: Star formation quenching stages of active and non-active galaxies

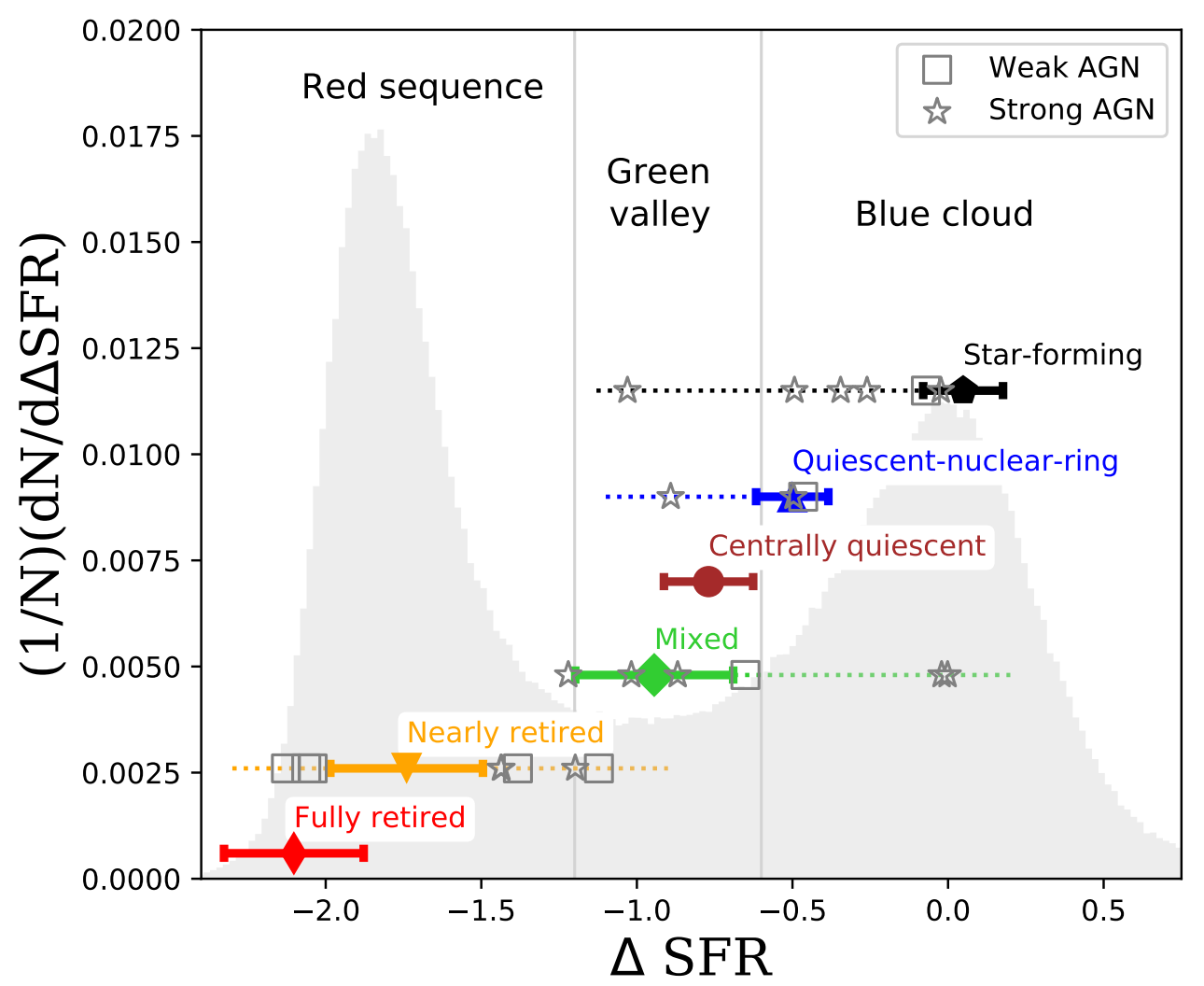

Fig. 11. Distribution of $\Delta$ SFR for SDSS DR7 galaxies at $z \sim 0$ (shaded regions; MPA-JHU catalogue, Kauffmann et al. 2003; Brinchmann et al. 2004; Salim et al. 2007) and for our sample of galaxies. The SDSS $y$-axis gives the number of the galaxies per given $\Delta$ SFR bin (i.e. d $N /$ d $\Delta$ SFR), normalised to the total number $N$ of the SDSS galaxies, while the $y$-axis of our sample is arbitrary and chosen here only for representative purposes (due to the smaller number of our galaxies in contrast to the number of the SDSS galaxies). The vertical grey lines indicate the green valley region (adopted values from Bluck et al. 2016). The colour symbols indicate the median $\Delta$ SFR distribution for the non-active galaxies of our sample in each QS, while the colour bar indicates the corresponding median absolute deviation. The active galaxies are represented by the grey empty symbols (stars for sAGNs and squares for wAGNs). The horizontal dotted colour lines are plotted to guide the eye for the position of the active galaxies in comparison to the non-active ones for a given QS. Quenching stage galaxies are well divided through $\Delta$ SFR, suggesting that the QS represents a certain evolutionary phase from the life cycle of the galaxy (see Sect. 6.1).

central spheroid could explain the precedence of the inside-out over the outside-in quenching scenario for the evolution of our galaxies (e.g. González Delgado et al. 2016; Ellison et al. 2018; Lin et al. 2019; Bluck et al. 2019).

The proposed EL classification is mostly based on the morphological distribution of the value of $W_{\mathrm{H} \alpha}$. This approach presents a unique perspective that allows us to define some EL classes as specific QSs. Nevertheless, we also include the BPT diagrams to search for signatures of AGN ionisation in the galaxies. In particular, while sAGNs are exclusively observed in the green valley of our sample (see also Kauffmann et al. 2003; Salim et al. 2007; Sánchez et al. 2018; Lacerda et al. 2020), galaxies hosting wAGNs are spread over the green valley and the red sequence. A similar bi-modality of the AGNs is also noted by Heckman \& Best (2014).

The role of the AGN in the quenching of the galaxies is complex and not yet well understood. AGNs can enhance or suppress star formation in galaxies (e.g. Wagner et al. 2016; Husemann \& Harrison 2018; Cresci \& Maiolino 2018). For instance, gas outflow from the AGN can trigger the formation of new stars in dense cold gas environments (the so-called positive feedback; Silk 2013; Zubovas et al. 2013; Mahoro et al. 2017). On the other hand, the suppression of star formation can happen via heating of the cold gas or its mechanical removal (the so-called negative feedback; Silk \& Rees 1998; Nesvadba et al. 2010; McNamara et al. 2016).
The negative feedback is well accepted in models to regulate massive galaxy growth (e.g. Kauffmann \& Haehnelt 2000; Schaye et al. 2015). For example, Frigo et al. (2019) investigate the impact of AGN feedback on the formation of massive galaxies through performance of cosmological simulations and find evidence that the AGN feedback plays an essential role in the formation of quiescent, slow-rotating, triaxial and non-discy early-type galaxies.

We find only a small percentage of AGN-host galaxies in our sample. Similar small percentages have been observed elsewhere, and this has been interpreted as evidence that starformation quenching in the nearby universe cannot be due to AGN action alone (e.g. Saintonge et al. 2012, 2017; Kirkpatrick et al. 2014). Some studies report that AGN feedback acts locally and does not influence the global SFR budget of the galaxy (e.g. Shin et al. 2019). However, these conclusions could be misleading, because the AGN may no longer be luminous when the effect from the AGN becomes observable (as discussed in Harrison 2017).

Surprisingly, the QSs of active and non-active galaxies seem almost identical (except of centrally quiescent and fully retired QSs, which only represent the non-active galaxy group as described in Sect. 3.1). Additionally, the analysis of active and non-active galaxies at the same QS allowed us to observe that there are no significant differences in the properties of objects with different nuclear activity. An exception is observed for 
the star-forming QS, where all AGNs (mainly strong AGNs) in this group are hosted by high-mass and older galaxies that are relatively close to the star-formation green valley. It seems that galaxies do not develop an observable AGN until several of their properties have reached some given value (e.g. the so-called golden mass, Dekel et al. 2019). Future exploration of the gas properties of the EL galaxies will shed more light on the relation between the role of the AGN feedback and the star-formation quenching of the galaxies.

\section{Summary}

We explore the ionised gas properties of 238 (E1-Sdm) CALIFA galaxies through 2D emission-line classification (QuestNA) based on spatially resolved $W_{\mathrm{H} \alpha}$ maps and BPT diagrams ([NI], [SII], [OI]). We define six quenching stages: galaxies dominated by recent star-formation; centrally quiescent galaxies; systems possessing quiescent-nuclear-ring-like structure; galaxies with mixed ionised processes; nearly retired galaxies that posses only a few star forming regions, and systems that are completely quiescent (fully retired within $2 R_{\mathrm{e}}$ ). We further divide the galaxies according to their nuclear activity: non-active galaxies, host of a weak (wAGN), or strong AGN (sAGN). The degree of star-formation quenching increases along the present EL-pattern sequence from star-forming to fully retired.

The EL classes occupy certain domains in the SFR- $M_{*}$ diagram, where star-forming and quiescent-nuclear-ring classes are located within the blue cloud; centrally quiescent and mixed are transient galaxies in the green valley; and nearly and fully retired are members of the red sequence.

The active galaxies do not show significantly different properties from their corresponding non-active counterparts for a given QS, except for the star-forming sAGN class. Overall, the sAGN galaxies are mainly located in the blue cloud or the green valley. On the other hand, the wAGN galaxies are largely dispersed between the green valley and the red sequence, and mostly occupy either the low-mass or high-mass end of the SFR$M_{*}$ diagram.

A morphological analysis of our sample indicates that secular evolutionary features (e.g. bar, spiral arms) dominate the structure of quiescent-nuclear-ring, centrally quiescent, and mixed classes in comparison to the other EL classes. The $W_{\mathrm{H} \alpha}$ profiles of the EL galaxies indicate an 'inside-out' star-formation quenching scenario and emphasise the importance of the galaxy bulge or central spheroid (see Fig. 6).

In forthcoming papers, we will continue the exploration of the CALIFA galaxies grouped in EL classes through various other properties, such as dynamics, velocity profiles, gas content, and environment, in order to complete the proposed classification and further explore the quenching mechanism of the active and non-active galaxies.

Acknowledgements. We thank the anonymous referee, whose suggestions helped us to improve the quality and presentation of this paper. DC acknowledges support by the Deutsche Forschungsgemeinschaft, DFG project number SFB956A. SFS acknowledge Conacyt projects CB-285080 and FC-2016-01 1916, and PAPIIT-DGAPA IN100519. RGB and RGD acknowledges financial support from the State Agency for Research of the Spanish MCIU through the "Center of Excellence Severo Ochoa" award to the Instituto de Astrofísica de Andalucía (SEV-2017-0709), and AYA2016-77846-P. ER acknowledges the support of the Natural Sciences and Engineering Research Council of Canada (NSERC), funding reference number RGPIN-2017-03987. In this study, we made use of the data of the first legacy survey, the Calar Alto Legacy Integral Field Area (CALIFA) survey, based on observations made at the Centro Astronómico Hispano Alemán (CAHA) at Calar Alto, operated jointly by the Max Planck-Institut für Astronomie and the Instituto de Astrofísica de Andalucía (CSIC). This research has made use of the NASA/IPAC Extragalactic Database (NED), which is operated by the Jet Propulsion Laboratory, California Institute of Technology, under contract with the National Aeronautics and Space Administration. Funding for the Sloan Digital Sky Survey IV has been provided by the Alfred P. Sloan Foundation, the US Department of Energy Office of Science, and the Participating Institutions. SDSS-IV acknowledges support and resources from the Center for High-Performance Computing at the University of Utah. The SDSS web site is www.sdss.org. SDSS-IV is managed by the Astrophysical Research Consortium for the Participating Institutions of the SDSS Collaboration including the Brazilian Participation Group, the Carnegie Institution for Science, Carnegie Mellon University, the Chilean Participation Group, the French Participation Group, Harvard-Smithsonian Center for Astrophysics, Instituto de Astrofísica de Canarias, The Johns Hopkins University, Kavli Institute for the Physics and Mathematics of the Universe (IPMU)/University of Tokyo, Lawrence Berkeley National Laboratory, Leibniz Institut für Astrophysik Potsdam (AIP), Max-Planck-Institut für Astronomie (MPIA Heidelberg), Max-Planck-Institut für Astrophysik (MPA Garching), Max-Planck-Institut für Extraterrestrische Physik (MPE), National Astronomical Observatory of China, New Mexico State University, New York University, University of Notre Dame, Observatário Nacional/MCTI, The Ohio State University, Pennsylvania State University, Shanghai Astronomical Observatory, United Kingdom Participation Group, Universidad Nacional Autónoma de México, University of Arizona, University of Colorado Boulder, University of Oxford, University of Portsmouth, University of Utah, University of Virginia, University of Washington, University of Wisconsin, Vanderbilt University, and Yale University. This research made use of Astropy (http: //www . astropy.org), a community-developed core Python package for Astronomy (Astropy Collaboration, 2013).

\section{References}

Abazajian, K. N., Adelman-McCarthy, J. K., Agüeros, M. A., et al. 2009, ApJS, 182,543

Alam, S., Albareti, F. D., Allende Prieto, C., et al. 2015, ApJS, 219, 12

Alongi, M., Bertelli, G., Bressan, A., et al. 1993, A\&AS, 97, 851

Aquino-Ortíz, E., Valenzuela, O., Sánchez, S. F., et al. 2018, MNRAS, 479, 2133

Baldry, I. K., Glazebrook, K., Brinkmann, J., et al. 2004, ApJ, 600, 681

Baldwin, A., Phillips, M. M., \& Terlevich, R. 1981, PASP, 93, 817

Barrera-Ballesteros, J. K., Heckman, T., Sánchez, S. F., et al. 2018, ApJ, 852, 74

Bekki, K. 2009, MNRAS, 399, 2221

Belfiore, F., Maiolino, R., Maraston, C., et al. 2016, MNRAS, 461, 3111

Belfiore, F., Maiolino, R., Tremonti, C., et al. 2017, MNRAS, 469, 151

Belfiore, F., Maiolino, R., Bundy, K., et al. 2018, MNRAS, 477, 3014

Blanton, M. R., \& Moustakas, J. 2009, ARA\&A, 47, 159

Bluck, A. F. L., Mendel, J. T., Ellison, S. L., et al. 2016, MNRAS, 462, 2559

Bluck, A. F. L., Bottrell, C., Teimoorinia, H., et al. 2019, MNRAS, 485, 666

Bluck, A. F. L., Maiolino, R., Sánchez, S. F., et al. 2020, MNRAS, 492, 96

Bolatto, A. D., Wong, T., Utomo, D., et al. 2017, ApJ, 846, 159

Brammer, G. B., Whitaker, K. E., van Dokkum, P. G., et al. 2009, ApJ, 706, L173

Bressan, A., Fagotto, F., Bertelli, G., \& Chiosi, C. 1993, A\&AS, 100, 647

Brinchmann, J., Charlot, S., White, S. D. M., et al. 2004, MNRAS, 351, 1151

Bruzual, G., \& Charlot, S. 2003, MNRAS, 344, 1000

Bundy, K., Bershady, M. A., Law, D. R., et al. 2015, ApJ, 798, 7

Cano-Díaz, M., Sánchez, S. F., Zibetti, S., et al. 2016, ApJ, 821, L26

Cano-Díaz, M., Ávila-Reese, V., Sánchez, S. F., et al. 2019, MNRAS, 488, 3929

Cappellari, M. 2008, MNRAS, 390, 71

Cappellari, M., \& Copin, Y. 2003, MNRAS, 342, 345

Cappellari, M., \& Emsellem, E. 2004, PASP, 116, 138

Cappellari, M., Emsellem, E., Krajnović, D., et al. 2011, MNRAS, 413, 813

Cappellari, M., McDermid, R. M., Alatalo, K., et al. 2012, Nature, 484, 485

Catalán-Torrecilla, C., Gil de Paz, A., Castillo-Morales, A., et al. 2015, A\&A, 584, A87

Catalán-Torrecilla, C., Gil de Paz, A., Castillo-Morales, A., et al. 2017, ApJ, 848, 87

Chabrier, G. 2003, PASP, 115, 763

Charbonnel, C., Meynet, G., Maeder, A., Schaller, G., \& Schaerer, D. 1993, A\&AS, 101, 415

Cid Fernandes, R., Mateus, A., Sodré, L., Stasińska, G., \& Gomes, J. M. 2005, MNRAS, 358, 363

Cid Fernandes, R., Stasińska, G., Schlickmann, M. S., et al. 2010, MNRAS, 403, 1036

Cid Fernandes, R., Stasińska, G., Mateus, A., \& Vale Asari, N. 2011, MNRAS, 413, 1687

Cid Fernandes, R., Pérez, E., García Benito, R., et al. 2013, A\&A, 557, A86

Cid Fernandes, R., González Delgado, R. M., García Benito, R., et al. 2014, A\&A, 561, A130

Colombo, D., Sanchez, S. F., Bolatto, A. D., et al. 2020, A\&A, 644, A97

Cresci, G., \& Maiolino, R. 2018, Nat. Astron., 2, 179

Croom, S. M., Lawrence, J. S., Bland-Hawthorn, J., et al. 2012, MNRAS, 421, 872 
Das, M., McGaugh, S. S., Ianjamasimanana, R., Schombert, J., \& Dwarakanath, K. S. 2020, ApJ, 889, 10

de Amorim, A. L., García-Benito, R., Cid Fernandes, R., et al. 2017, MNRAS, 471,3727

Dekel, A., Lapiner, S., \& Dubois, Y. 2019, ArXiv e-prints [arXiv:1904.08431]

Elbaz, D., Daddi, E., Le Borgne, D., et al. 2007, A\&A, 468, 33

Ellison, S. L., Sánchez, S. F., Ibarra-Medel, H., et al. 2018, MNRAS, 474, 2039

Emsellem, E., Monnet, G., \& Bacon, R. 1994, A\&A, 285, 723

Emsellem, E., Cappellari, M., Krajnović, D., et al. 2007, MNRAS, 379, 401

Espinosa-Ponce, C., Sánchez, S. F., Morisset, C., et al. 2020, MNRAS, 494, 1622

Faber, S. M., Willmer, C. N. A., Wolf, C., et al. 2007, ApJ, 665, 265

Fagotto, F., Bressan, A., Bertelli, G., \& Chiosi, C. 1994a, A\&AS, 104, 365

Fagotto, F., Bressan, A., Bertelli, G., \& Chiosi, C. 1994b, A\&AS, 105, 29

Fagotto, F., Bressan, A., Bertelli, G., \& Chiosi, C. 1994c, A\&AS, 105, 39

Falcón-Barroso, J., Sánchez-Blázquez, P., Vazdekis, A., et al. 2011, A\&A, 532, A95

Falcón-Barroso, J., Lyubenova, M., van de Ven, G., et al. 2017, A\&A, 597, A48

Falcón-Barroso, J., van de Ven, G., Lyubenova, M., et al. 2019, A\&A, 632, A59

Flores-Fajardo, N., Morisset, C., Stasińska, G., \& Binette, L. 2011, MNRAS, 415,2182

Foreman-Mackey, D., Hogg, D. W., Lang, D., \& Goodman, J. 2013, PASP, 125 306

Frigo, M., Naab, T., Hirschmann, M., et al. 2019, MNRAS, 489, 2702

Galbany, L., Anderson, J. P., Sánchez, S. F., et al. 2018, ApJ, 855, 107

Gallazzi, A., Brinchmann, J., Charlot, S., \& White, S. D. M. 2008, MNRAS, 383,1439

Gallazzi, A., Charlot, S., Brinchmann, J., White, S. D. M., \& Tremonti, C. A. 2005, MNRAS, 362, 41

García-Benito, R., Zibetti, S., Sánchez, S. F., et al. 2015, A\&A, 576, A135

García-Benito, R., González Delgado, R. M., Pérez, E., et al. 2017, A\&A, 608, A27

García-Benito, R., González Delgado, R. M., Pérez, E., et al. 2019, A\&A, 621, A 120

Girardi, L., Bressan, A., Chiosi, C., Bertelli, G., \& Nasi, E. 1996, A\&AS, 117, 113

Girardi, L., Bressan, A., Bertelli, G., \& Chiosi, C. 2000, A\&AS, 141, 371

González Delgado, R. M., García-Benito, R., Pérez, E., et al. 2015, A\&A, 581, A103

González Delgado, R. M., Cid Fernandes, R., Pérez, E., et al. 2016, A\&A, 590, A44

González Delgado, R. M., Pérez, E., Cid Fernandes, R., et al. 2017, A\&A, 607, A128

Häring, N., \& Rix, H.-W. 2004, ApJ, 604, L89

Harrison, C. M. 2017, Nat. Astron., 1, 0165

Heckman, T. M., \& Best, P. N. 2014, ARA\&A, 52, 589

Hoversten, E. A., \& Glazebrook, K. 2008, ApJ, 675, 163

Hubble, E. P. 1926, ApJ, 64, 321

Husemann, B., \& Harrison, C. M. 2018, Nat. Astron., 2, 196

Husemann, B., Jahnke, K., Sánchez, S. F., et al. 2013, A\&A, 549, A87

Kalinova, V., Colombo, D., Rosolowsky, E., et al. 2017a, MNRAS, 469, 2539

Kalinova, V., van de Ven, G., Lyubenova, M., et al. 2017b, MNRAS, 464, 1903

Kauffmann, G., \& Haehnelt, M. 2000, MNRAS, 311, 576

Kauffmann, G., Heckman, T. M., White, S. D. M., et al. 2003, MNRAS, 341, 33

Kelz, A., Verheijen, M. A. W., Roth, M. M., et al. 2006, PASP, 118, 129

Kennicutt, R. C., \& Evans, N. J. 2012, ARA\&A, 50, 531

Kewley, L. J., Dopita, M. A., Sutherland, R. S., Heisler, C. A., \& Trevena, J. 2001, ApJ, 556, 121

Kewley, L. J., Groves, B., Kauffmann, G., \& Heckman, T. 2006, MNRAS, 372, 961

Kirkpatrick, A., Pope, A., Aretxaga, I., et al. 2014, ApJ, 796, 135

Lacerda, E. A. D., Cid Fernandes, R., Couto, G. S., et al. 2018, MNRAS, 474 3727

Lacerda, E. A. D., Sánchez, S. F., Cid Fernandes, R., et al. 2020, MNRAS, 492, 3073

Le Borgne, J. F., Bruzual, G., Pelló, R., et al. 2003, A\&A, 402, 433

Leung, G. Y. C., Leaman, R., van de Ven, G., et al. 2018, MNRAS, 477, 254

Lin, L., Belfiore, F., Pan, H.-A., et al. 2017, ApJ, 851, 18

Lin, L., Hsieh, B.-C., Pan, H.-A., et al. 2019, ApJ, 872, 50

López-Cobá, C., Sánchez, S. F., Bland-Hawthorn, J., et al. 2019, MNRAS, 482, 4032

López-Cobá, C., Sánchez, S. F., Anderson, J. P., et al. 2020, AJ, 159, 167

Lyubenova, M., Martín-Navarro, I., van de Ven, G., et al. 2016, MNRAS, 463, 3220

Mahoro, A., Pović, M., \& Nkundabakura, P. 2017, MNRAS, 471, 3226

Marconi, A., \& Hunt, L. K. 2003, ApJ, 589, L21

Martig, M., Bournaud, F., Teyssier, R., \& Dekel, A. 2009, ApJ, 707, 250

Martins, L. P., González Delgado, R. M., Leitherer, C., Cerviño, M., \& Hauschildt, P. 2005, MNRAS, 358, 49
McConnell, N. J., \& Ma, C.-P. 2013, ApJ, 764, 184

McGaugh, S. S., Schombert, J. M., Bothun, G. D., \& de Blok, W. J. G. 2000, ApJ, 533, L99

McNamara, B. R., Russell, H. R., Nulsen, P. E. J., et al. 2016, ApJ, 830, 79

Méndez-Abreu, J., Ruiz-Lara, T., Sánchez-Menguiano, L., et al. 2017, A\&A, 598, A32

Nesvadba, N. P. H., Boulanger, F., Salomé, P., et al. 2010, A\&A, 521, A65

Renzini, A., \& Peng, Y.-J. 2015, ApJ, 801, L29

Roth, M. M., Kelz, A., Fechner, T., et al. 2005, PASP, 117, 620

Saintonge, A., Tacconi, L. J., Fabello, S., et al. 2012, ApJ, 758, 73

Saintonge, A., Catinella, B., Tacconi, L. J., et al. 2017, ApJS, 233, 22

Salim, S., Rich, R. M., Charlot, S., et al. 2007, ApJS, 173, 267

Salpeter, E. E. 1955, A\&A, 121, 161

Sánchez, S. F. 2020, ARA\&A, 58, 99

Sánchez, S. F., Kennicutt, R. C., Gil de Paz, A., et al. 2012, A\&A, 538, A8

Sánchez, S. F., Rosales-Ortega, F. F., Jungwiert, B., et al. 2013, A\&A, 554, A58

Sánchez, S. F., Rosales-Ortega, F. F., Iglesias-Páramo, J., et al. 2014, A\&A, 563, A49

Sánchez, S. F., García-Benito, R., Zibetti, S., et al. 2016a, A\&A, 594, A36

Sánchez, S. F., Pérez, E., Sánchez-Blázquez, P., et al. 2016b, Rev. Mexicana Astron. Astrofis., 52, 171

Sánchez, S. F., Pérez, E., Sánchez-Blázquez, P., et al. 2016c, Rev. Mexicana Astron. Astrofis., 52, 21

Sánchez, S. F., Avila-Reese, V., Hernandez-Toledo, H., et al. 2018, Rev. Mexicana Astron. Astrofis., 54, 217

Sánchez, S. F., Avila-Reese, V., Rodríguez-Puebla, A., et al. 2019, MNRAS, 482, 1557

Sánchez-Blázquez, P., Peletier, R. F., Jiménez-Vicente, J., et al. 2006, MNRAS, 371, 703

Sánchez-Menguiano, L., Sánchez, S. F., Pérez, I., et al. 2016, A\&A, 587, A70

Sánchez-Menguiano, L., Sánchez, S. F., Pérez, I., et al. 2018, A\&A, 609, A119

Sarzi, M., Shields, J. C., Schawinski, K., et al. 2010, MNRAS, 402, 2187

Schaerer, D., Charbonnel, C., Meynet, G., Maeder, A., \& Schaller, G. 1993a, A\&AS, 102, 339

Schaerer, D., Meynet, G., Maeder, A., \& Schaller, G. 1993b, A\&AS, 98, 523

Schaller, G., Schaerer, D., Meynet, G., \& Maeder, A. 1992, A\&AS, 96, 269

Schawinski, K., Thomas, D., Sarzi, M., et al. 2007, MNRAS, 382, 1415

Schawinski, K., Urry, C. M., Simmons, B. D., et al. 2014, MNRAS, 440, 889

Schaye, J., Crain, R. A., Bower, R. G., et al. 2015, MNRAS, 446, 521

Shin, J., Woo, J.-H., Chung, A., et al. 2019, ApJ, 881, 147

Silk, J. 2013, ApJ, 772, 112

Silk, J., \& Rees, M. J. 1998, A\&A, 331, L1

Singh, R., van de Ven, G., Jahnke, K., et al. 2013, A\&A, 558, A43

Speagle, J. S., Steinhardt, C. L., Capak, P. L., \& Silverman, J. D. 2014, ApJS, 214,15

Stasińska, G., Vale Asari, N., Cid Fernandes, R., et al. 2008, MNRAS, 391, L29

Strateva, I., Ivezić, Ž., Knapp, G. R., et al. 2001, AJ, 122, 1861

Struve, C., Oosterloo, T., Sancisi, R., Morganti, R., \& Emonts, B. H. C. 2010 , A\&A, 523, A75

Thorp, M. D., Ellison, S. L., Simard, L., Sánchez, S. F., \& Antonio, B. 2019, MNRAS, 482, L55

Tukey, J. W. 1977, Exploratory data analysis (Addison-Wesley Publishing Company)

Valdes, F., Gupta, R., Rose, J. A., Singh, H. P., \& Bell, D. J. 2004, ApJS, 152, 251

Vazdekis, A., Sánchez-Blázquez, P., Falcón-Barroso, J., et al. 2010, MNRAS 404, 1639

Veilleux, S., \& Osterbrock, D. E. 1987, ApJS, 63, 295

Verheijen, M. A. W., Bershady, M. A., Andersen, D. R., et al. 2004, Astron. Nachr., 325, 151

Vulcani, B., Fritz, J., Poggianti, B. M., et al. 2020, ApJ, 892, 146

Wagner, A. Y., Bicknell, G. V., Umemura, M., Sutherland, R. S., \& Silk, J. 2016 Astrono. Nachr., 337, 167

Walcher, C. J., Wisotzki, L., Bekeraité, S., et al. 2014, A\&A, 569, A1

Weiner, B. J., Willmer, C. N. A., Faber, S. M., et al. 2006, ApJ, 653, 1027

Whitaker, K. E., van Dokkum, P. G., Brammer, G., \& Franx, M. 2012, ApJ, 754, L 29

Williams, R. J., Quadri, R. F., Franx, M., van Dokkum, P., \& Labbé, I. 2009, ApJ, 691, 1879

Young, J. S., \& Scoville, N. Z. 1991, ARA\&A, 29, 581

Zhu, L., van de Ven, G., van den Bosch, R., et al. 2018a, Nat. Astron., 2, 233

Zhu, L., van de Ven, G., Méndez-Abreu, J., \& Obreja, A. 2018b, MNRAS, 479 945

Zibetti, S., Gallazzi, A. R., Ascasibar, Y., et al. 2017, MNRAS, 468, 1902

Zubovas, K., Nayakshin, S., King, A., \& Wilkinson, M. 2013, MNRAS, 433 3079 


\section{Appendix A: Diagnostic maps and diagrams for all 238 CALIFA galaxies}

The resolved $W_{\mathrm{H} \alpha}$ maps, $W_{\mathrm{H} \alpha}$ profiles, BPT maps, and BPT diagrams for the full sample of 238 CALIFA galaxies are available at https://doi .org/10.5281/zenodo. 4529149.

\section{Appendix B: Tables with properties of the 238 CALIFA galaxies}

In Tables B.1 and B.2, we list the main properties of the 238 (E1-Sdm) CALIFA galaxies, presented in this work.

Table B.1. Properties of the 238 (E1-Sdm) CALIFA galaxies.

\begin{tabular}{|c|c|c|c|c|c|c|c|c|c|c|c|}
\hline $\begin{array}{l}\text { Galaxy } \\
\text { (1) }\end{array}$ & $\begin{array}{c}D \\
\mathrm{Mpc} \\
(2) \\
\end{array}$ & $\begin{array}{c}\mathrm{Re} \\
\operatorname{arcsec} \\
(3)\end{array}$ & $\begin{array}{c}\log M_{*} \\
M_{\odot} \\
(4)\end{array}$ & $\begin{array}{c}\log \mathrm{SFR} \\
M_{\odot} \mathrm{yr}^{-1} \\
(5)\end{array}$ & $\begin{array}{c}\Delta \mathrm{SFR} \\
M_{\odot} \mathrm{yr}^{-1} \\
(6)\end{array}$ & $\begin{array}{l}\text { Type } \\
(7)\end{array}$ & $\begin{array}{l}\text { Bar } \\
(8) \\
\end{array}$ & $\begin{array}{l}\text { QS } \\
\text { (9) }\end{array}$ & $\begin{array}{c}\text { flag-QS } \\
(10)\end{array}$ & $\begin{array}{l}\text { NA } \\
\text { (11) } \\
\end{array}$ & $\begin{array}{c}\text { flag-NA } \\
\text { (12) }\end{array}$ \\
\hline IC0480 & 61.7 & 14.0 & 9.988 & 0.145 & -0.016 & $\mathrm{Sc}$ & $\mathrm{AB}$ & SF & 1 & nonA & S \\
\hline IC0540 & 26.1 & 12.1 & 9.736 & -0.903 & -0.869 & Sab & $\mathrm{AB}$ & MX & 1 & sAGN & S \\
\hline IC0674 & 103.4 & 9.2 & 10.997 & -0.096 & -1.033 & $\mathrm{Sab}$ & B & MX & 2 & nonA & S \\
\hline IC0944 & 95.8 & 13.6 & 11.108 & 0.026 & -0.996 & $\mathrm{Sab}$ & A & MX & 1 & nonA & S \\
\hline IC 1079 & 119.3 & 18.2 & 11.252 & -0.628 & -1.762 & E4 & A & $\mathrm{nR}$ & 2 & nonA & S \\
\hline IC 1151 & 31.0 & 19.9 & 9.521 & 0.006 & 0.206 & Scd & B & SF & 1 & nonA & S \\
\hline IC 1256 & 67.0 & 10.1 & 10.397 & 0.157 & -0.319 & $\mathrm{Sb}$ & $\mathrm{AB}$ & SF & 1 & nonA & $S$ \\
\hline IC 1528 & 53.3 & 16.0 & 10.134 & 0.428 & 0.155 & Sbc & $\mathrm{AB}$ & SF & 1 & nonA & $S$ \\
\hline IC1652 & 72.9 & 8.3 & 10.502 & -1.479 & -2.035 & SOa & A & $\mathrm{fR}$ & 1 & nonA & $S$ \\
\hline IC 1755 & 109.9 & 16.4 & 10.977 & -0.507 & -1.429 & $\mathrm{Sb}$ & A & MX & 1 & nonA & S \\
\hline IC2101 & 60.7 & 15.8 & 10.164 & 0.425 & 0.130 & Scd & $\mathrm{AB}$ & $\mathrm{SF}$ & 1 & nonA & $S$ \\
\hline IC2247 & 57.7 & 15.2 & 10.429 & 0.154 & -0.345 & $\mathrm{Sab}$ & A & SF & 1 & sAGN & $S$ \\
\hline IC2487 & 58.3 & 15.8 & 10.326 & 0.202 & -0.219 & $\mathrm{Sc}$ & $\mathrm{AB}$ & SF & 1 & nonA & S \\
\hline IC4566 & 81.0 & 12.7 & 10.779 & -0.025 & -0.794 & $\mathrm{Sb}$ & B & MX & 1 & nonA & S \\
\hline IC5309 & 59.6 & 15.9 & 10.109 & 0.164 & -0.089 & $\mathrm{Sc}$ & $\mathrm{AB}$ & $\mathrm{SF}$ & 2 & nonA & $S$ \\
\hline IC5376 & 71.5 & 11.1 & 10.584 & -0.254 & -0.873 & $\mathrm{Sb}$ & A & $\mathrm{cQ}$ & 1 & nonA & $S$ \\
\hline MCG-01-54-016 & 42.1 & 16.8 & 9.156 & -0.528 & -0.048 & Scd & A & $\mathrm{SF}$ & 1 & nonA & S \\
\hline MCG-02-02-030 & 48.9 & 12.4 & 10.291 & -0.103 & -0.497 & $\mathrm{Sb}$ & $\mathrm{AB}$ & QnR & 1 & sAGN & $\mathrm{U}$ \\
\hline MCG-02-02-040 & 49.8 & 16.1 & 9.952 & 0.108 & -0.025 & Scd & $\mathrm{AB}$ & $\mathrm{SF}$ & 1 & nonA & $S$ \\
\hline MCG-02-03-015 & 79.8 & 12.7 & - & - & - & $\mathrm{Sab}$ & $\mathrm{AB}$ & SF & 3 & nonA & S \\
\hline MCG-02-51-004 & 79.3 & 11.6 & 10.650 & 0.402 & -0.268 & $\mathrm{Sb}$ & A & SF & 1 & nonA & $S$ \\
\hline NGC 0001 & 64.7 & 11.7 & 10.691 & 0.494 & -0.207 & $\mathrm{Sbc}$ & A & SF & 1 & nonA & S \\
\hline NGC 0023 & 64.9 & 7.8 & 10.935 & 0.983 & 0.094 & $\mathrm{Sb}$ & B & $\mathrm{SF}$ & 1 & nonA & $\mathrm{S}$ \\
\hline NGC 0155 & 85.8 & 9.2 & 10.884 & -1.809 & -2.659 & E1 & A & $\mathrm{fR}$ & 1 & nonA & S \\
\hline NGC 0160 & 74.0 & 16.8 & 11.047 & 0.050 & -0.926 & $\mathrm{Sa}$ & A & $\mathrm{cQ}$ & 2 & nonA & $S$ \\
\hline NGC 0171 & 53.9 & 18.0 & 10.641 & -0.228 & -0.890 & $\mathrm{Sb}$ & B & $\mathrm{cQ}$ & 1 & nonA & S \\
\hline NGC 0177 & 52.7 & 16.2 & - & - & - & Sab & A & QnR & 2 & nonA & S \\
\hline NGC 0192 & 57.9 & 14.2 & 10.750 & 0.348 & -0.399 & $\mathrm{Sab}$ & $\mathrm{AB}$ & $\mathrm{MX}$ & 1 & nonA & $\mathrm{S}$ \\
\hline NGC 0214 & 64.2 & 14.8 & 10.801 & 0.715 & -0.071 & Sbc & $\mathrm{AB}$ & $\mathrm{SF}$ & 1 & wAGN & $\mathrm{S}$ \\
\hline NGC 0216 & 21.4 & 10.9 & 9.185 & -0.404 & 0.054 & $\mathrm{Sd}$ & A & $\mathrm{SF}$ & 1 & nonA & $\mathrm{S}$ \\
\hline NGC 0217 & 55.2 & 19.2 & 10.832 & -0.258 & -1.068 & $\mathrm{Sa}$ & A & MX & 1 & nonA & $S$ \\
\hline NGC 0237 & 58.4 & 10.1 & 10.195 & 0.507 & 0.187 & $\mathrm{Sc}$ & B & SF & 1 & nonA & $\mathrm{S}$ \\
\hline NGC 0257 & 73.7 & 16.2 & 10.842 & 0.941 & 0.124 & $\mathrm{Sc}$ & A & SF & 1 & nonA & $\mathrm{S}$ \\
\hline NGC 0429 & 78.0 & 6.7 & 10.736 & -1.448 & -2.184 & $\mathrm{Sa}$ & A & $\mathrm{fR}$ & 1 & nonA & $S$ \\
\hline NGC 0444 & 68.3 & 15.2 & 9.794 & 0.090 & 0.080 & Scd & A & SF & 1 & nonA & $\mathrm{S}$ \\
\hline NGC 0499 & 62.3 & 9.2 & 11.240 & -1.140 & -2.265 & E5 & A & $\mathrm{fR}$ & 1 & nonA & $\mathrm{S}$ \\
\hline NGC 0504 & 59.9 & 6.8 & 10.587 & -1.410 & -2.032 & So & A & fR & 1 & nonA & $\mathrm{S}$ \\
\hline NGC 0517 & 59.6 & 5.8 & 10.644 & -1.509 & -2.175 & So & A & $\mathrm{fR}$ & 1 & nonA & $\mathrm{S}$ \\
\hline NGC 0528 & 67.8 & 8.2 & 10.932 & -0.970 & -1.857 & S0 & A & $\mathrm{nR}$ & 1 & nonA & $S$ \\
\hline NGC 0529 & 68.1 & 9.9 & 11.079 & -0.977 & -1.977 & E4 & A & $\mathrm{fR}$ & 1 & nonA & $S$ \\
\hline
\end{tabular}

Notes. Columns list: (1) Galaxy identifier (NED); (2) Galactocentric galaxy distance in Mpc (NED); (3) Effective radius of the galaxy in arcseconds, measured from SDSS images via growth curve analysis using elliptical apertures (Sanchez et al., in prep.); (4) Stellar mass, adopting Chabrier IMF in $M_{\odot}$ (Sect. 2.3); (5) SFR of the galaxies in $M_{\odot} \mathrm{yr}^{-1}$ (Sect. 2.3); (6) SFR distance, measured from the SFMS in $M_{\odot} \mathrm{yr}^{-1}$ (Sect. 6.1); (7) Hubble type based on by-eye morphological classification from Walcher et al. (2014); (8) Bar class based on by-eye morphological classification from Walcher et al. (2014), where A-non-barred galaxy, B-barred galaxy and AB - unsure bar in the galaxy (Sect. 5.1); (9) Quenching stages (QS) of the 238 CALIFA galaxies, where SF - Star-forming, QnR - quiescent-nuclear-ring; cQ - centrally quiescent; MX - mixed; nR nearly retired and fR - fully retired (Sect. 3); (10) Reliability of the QS classification, where 1 - sure class , 2 - unsure class due to complex structure of the galaxy, 3 - unsure class due to poor data (Sect. 3.3). (11) Nuclear activity groups for our sample, where nonA - non-active galaxy, wAGN-weak AGN and sAGN - strong AGN (Sect. 3.2). (12) Reliability of the NA classification, where S: sure class based on the information of the three BPT diagrams and U: unsure class based on the information of two from the three BPT diagrams (Sect. 3.3). 
Table B.1. continued.

\begin{tabular}{|c|c|c|c|c|c|c|c|c|c|c|c|}
\hline $\begin{array}{l}\text { Galaxy } \\
\text { (1) }\end{array}$ & $\begin{array}{c}D \\
\mathrm{Mpc} \\
(2) \\
\end{array}$ & $\begin{array}{c}\mathrm{Re} \\
\operatorname{arcsec} \\
(3) \\
\end{array}$ & $\begin{array}{c}\log M_{*} \\
M_{\odot} \\
(4)\end{array}$ & $\begin{array}{c}\log \mathrm{SFR} \\
M_{\odot} \mathrm{yr}^{-1} \\
(5)\end{array}$ & $\begin{array}{c}\Delta \mathrm{SFR} \\
M_{\odot} \mathrm{yr}^{-1} \\
(6)\end{array}$ & $\begin{array}{l}\text { Type } \\
(7) \\
\end{array}$ & $\begin{array}{l}\text { Bar } \\
(8) \\
\end{array}$ & $\begin{array}{l}\text { QS } \\
(9) \\
\end{array}$ & $\begin{array}{c}\text { flag-QS } \\
(10)\end{array}$ & $\begin{array}{l}\text { NA } \\
(11) \\
\end{array}$ & $\begin{array}{c}\text { flag-NA } \\
(12)\end{array}$ \\
\hline NGC 0551 & 73.2 & 15.1 & 10.565 & 0.200 & -0.404 & Sbc & $\mathrm{AB}$ & QnR & 1 & nonA & $\mathrm{S}$ \\
\hline NGC 0681 & 24.3 & 16.6 & 10.160 & -0.245 & -0.537 & $\mathrm{Sa}$ & $\mathrm{AB}$ & MX & 2 & nonA & $\mathrm{S}$ \\
\hline NGC 0741 & 77.0 & 15.4 & 11.434 & -0.839 & -2.113 & $\mathrm{E} 1$ & A & $\mathrm{nR}$ & 1 & nonA & $\mathrm{S}$ \\
\hline NGC 0755 & 22.6 & 17.8 & 9.300 & -0.298 & 0.072 & Scd & B & SF & 1 & nonA & $\mathrm{S}$ \\
\hline NGC 0768 & 96.7 & 15.2 & 10.579 & 0.450 & -0.165 & $\mathrm{Sc}$ & B & SF & 1 & nonA & $\mathrm{S}$ \\
\hline NGC 0774 & 64.4 & 7.4 & 10.763 & -0.992 & -1.749 & S0 & A & $\mathrm{nR}$ & 1 & nonA & $\mathrm{S}$ \\
\hline NGC 0776 & 68.8 & 13.2 & 10.803 & 0.382 & -0.406 & $\mathrm{Sb}$ & B & SF & 1 & nonA & $\mathrm{S}$ \\
\hline NGC 0781 & 48.7 & 7.2 & - & - & - & $\mathrm{Sa}$ & $\mathrm{A}$ & $\mathrm{fR}$ & 3 & nonA & $\mathrm{S}$ \\
\hline NGC 0810 & 106.0 & 10.3 & 11.357 & -0.350 & -1.564 & E5 & $\mathrm{A}$ & $\mathrm{nR}$ & 2 & nonA & $\mathrm{S}$ \\
\hline NGC 0932 & 56.9 & 14.0 & 10.957 & -0.454 & -1.360 & S0a & A & $\mathrm{nR}$ & 2 & nonA & $\mathrm{S}$ \\
\hline NGC 1056 & 22.4 & 8.0 & 10.017 & 0.093 & -0.090 & $\mathrm{Sa}$ & A & SF & 1 & nonA & $\mathrm{S}$ \\
\hline NGC 1060 & 72.5 & 13.0 & 11.472 & -0.654 & -1.957 & E3 & A & $\mathrm{fR}$ & 1 & nonA & $\mathrm{S}$ \\
\hline NGC 1167 & 69.1 & 13.4 & 11.231 & -0.320 & -1.437 & So & A & $\mathrm{nR}$ & 1 & sAGN & $\mathrm{U}$ \\
\hline NGC 1349 & 90.2 & 11.1 & 11.112 & -0.128 & -1.154 & E6 & A & MX & 2 & nonA & $\mathrm{S}$ \\
\hline NGC 1542 & 50.3 & 8.9 & 10.314 & 0.054 & -0.357 & $\mathrm{Sab}$ & $\mathrm{AB}$ & MX & 1 & nonA & $\mathrm{S}$ \\
\hline NGC 1645 & 65.9 & 12.2 & 10.764 & -0.481 & -1.239 & SOa & B & MX & 1 & nonA & $\mathrm{S}$ \\
\hline NGC 1677 & 36.5 & 15.2 & 9.467 & -0.195 & 0.046 & Scd & $\mathrm{AB}$ & $\mathrm{SF}$ & 1 & nonA & $\mathrm{S}$ \\
\hline NGC 2253 & 50.2 & 14.0 & 10.518 & 0.567 & -0.001 & $\mathrm{Sbc}$ & B & SF & 1 & nonA & $\mathrm{S}$ \\
\hline NGC 2347 & 61.9 & 12.0 & 10.681 & 0.653 & -0.041 & $\mathrm{Sbc}$ & $\mathrm{AB}$ & SF & 1 & nonA & $\mathrm{S}$ \\
\hline NGC 2410 & 63.7 & 16.1 & 10.767 & 0.267 & -0.494 & $\mathrm{Sb}$ & $\mathrm{AB}$ & SF & 1 & sAGN & $\mathrm{S}$ \\
\hline NGC 2449 & 66.3 & 11.9 & 10.854 & -0.013 & -0.840 & $\mathrm{Sab}$ & $\mathrm{AB}$ & MX & 1 & nonA & $\mathrm{S}$ \\
\hline NGC 2476 & 51.0 & 6.9 & 10.673 & -0.771 & -1.458 & E6 & A & $\mathrm{fR}$ & 1 & nonA & $\mathrm{S}$ \\
\hline NGC 2481 & 28.6 & 14.8 & 10.542 & -1.515 & -2.102 & S0 & A & MX & 1 & nonA & $\mathrm{S}$ \\
\hline NGC 2486 & 62.8 & 19.4 & - & - & - & $\mathrm{Sab}$ & B & MX & 1 & nonA & $\mathrm{S}$ \\
\hline NGC 2553 & 63.5 & 8.6 & 10.591 & -1.038 & -1.662 & $\mathrm{Sb}$ & $\mathrm{AB}$ & $\mathrm{fR}$ & 1 & nonA & $\mathrm{S}$ \\
\hline NGC 2554 & 56.0 & 15.3 & 11.032 & -0.418 & -1.382 & SOa & A & $\mathrm{nR}$ & 1 & wAGN & $\mathrm{S}$ \\
\hline NGC 2592 & 26.2 & 6.8 & 10.234 & -2.309 & -2.659 & $\mathrm{E} 4$ & $\mathrm{~A}$ & $\mathrm{fR}$ & 1 & nonA & $\mathrm{S}$ \\
\hline NGC 2604 & 27.8 & 18.0 & 9.343 & -0.226 & 0.111 & $\mathrm{Sd}$ & B & SF & 1 & nonA & $\mathrm{S}$ \\
\hline NGC 2639 & 46.1 & 10.9 & 10.853 & -0.191 & -1.018 & $\mathrm{Sa}$ & $\mathrm{A}$ & MX & 1 & sAGN & $\mathrm{S}$ \\
\hline NGC 2730 & 51.1 & 18.2 & 9.931 & 0.347 & 0.231 & Scd & B & $\mathrm{SF}$ & 1 & nonA & $\mathrm{S}$ \\
\hline NGC 2880 & 22.4 & 10.0 & 10.324 & -2.292 & -2.710 & E7 & $\mathrm{AB}$ & $\mathrm{fR}$ & 1 & nonA & $\mathrm{S}$ \\
\hline NGC 2906 & 27.6 & 12.2 & 10.261 & -0.098 & -0.469 & $\mathrm{Sbc}$ & $\mathrm{A}$ & $\mathrm{cQ}$ & 2 & nonA & $\mathrm{S}$ \\
\hline NGC 2916 & 50.0 & 18.7 & 10.455 & 0.314 & -0.206 & $\mathrm{Sbc}$ & A & $\mathrm{cQ}$ & 1 & nonA & $\mathrm{S}$ \\
\hline NGC 2918 & 93.1 & 7.3 & 10.986 & -1.247 & -2.176 & E6 & A & $\mathrm{fR}$ & 1 & nonA & $\mathrm{S}$ \\
\hline NGC 3057 & 22.9 & 18.5 & 8.775 & -0.806 & -0.033 & Sdm & B & SF & 1 & nonA & $\mathrm{S}$ \\
\hline NGC 3106 & 84.5 & 11.8 & 11.088 & -0.144 & -1.151 & $\mathrm{Sab}$ & $\mathrm{A}$ & MX & 2 & nonA & $\mathrm{S}$ \\
\hline NGC 3160 & 94.7 & 11.0 & 10.706 & -0.508 & -1.220 & $\mathrm{Sab}$ & $\mathrm{AB}$ & MX & 1 & sAGN & $\mathrm{S}$ \\
\hline NGC 3300 & 40.9 & 10.1 & 10.484 & -1.560 & -2.102 & S0a & B & $\mathrm{fR}$ & 1 & nonA & $\mathrm{S}$ \\
\hline NGC 3381 & 22.1 & 16.2 & 9.365 & -0.143 & 0.177 & $\mathrm{Sd}$ & B & $\mathrm{SF}$ & 1 & nonA & $\mathrm{S}$ \\
\hline NGC 3615 & 90.9 & 6.3 & 11.216 & -0.837 & -1.943 & E5 & A & $\mathrm{fR}$ & 1 & nonA & $\mathrm{S}$ \\
\hline NGC 3811 & 43.2 & 15.3 & 10.304 & 0.202 & -0.201 & $\mathrm{Sbc}$ & B & QnR & 1 & nonA & $\mathrm{S}$ \\
\hline NGC 3815 & 50.4 & 10.0 & 10.406 & 0.649 & 0.167 & $\mathrm{Sbc}$ & $\mathrm{A}$ & SF & 1 & nonA & $\mathrm{S}$ \\
\hline NGC 3994 & 42.3 & 5.7 & 10.314 & 0.598 & 0.187 & $\mathrm{Sbc}$ & $\mathrm{AB}$ & SF & 1 & nonA & $\mathrm{S}$ \\
\hline NGC 4003 & 88.7 & 8.7 & 10.959 & -0.156 & -1.063 & SOa & B & MX & 2 & nonA & $\mathrm{S}$ \\
\hline NGC 4047 & 47.5 & 12.9 & 10.506 & 0.620 & 0.060 & $\mathrm{Sbc}$ & $\mathrm{A}$ & $\mathrm{SF}$ & 1 & nonA & $\mathrm{S}$ \\
\hline NGC 4149 & 43.1 & 11.7 & 10.202 & -0.373 & -0.698 & $\mathrm{Sa}$ & $\mathrm{AB}$ & MX & 1 & nonA & $\mathrm{S}$ \\
\hline NGC 4185 & 53.4 & 22.4 & 10.555 & -0.030 & -0.626 & $\mathrm{Sbc}$ & $\mathrm{AB}$ & $\mathrm{cQ}$ & 1 & nonA & $\mathrm{S}$ \\
\hline NGC 4210 & 39.0 & 19.2 & 10.180 & -0.033 & -0.341 & $\mathrm{Sb}$ & B & $\mathrm{cQ}$ & 1 & nonA & $\mathrm{S}$ \\
\hline NGC 4470 & 31.1 & 12.0 & 9.781 & 0.202 & 0.201 & Sc & $\mathrm{A}$ & SF & 1 & nonA & $\mathrm{S}$ \\
\hline NGC 4644 & 69.0 & 13.4 & 10.410 & -0.024 & -0.510 & $\mathrm{Sb}$ & A & $\mathrm{cQ}$ & 1 & nonA & $\mathrm{S}$ \\
\hline NGC 4711 & 56.1 & 12.5 & 10.511 & 0.672 & 0.109 & $\mathrm{Sbc}$ & A & SF & 1 & nonA & $\mathrm{S}$ \\
\hline NGC 4816 & 94.9 & 10.3 & 11.067 & -0.605 & -1.596 & E1 & A & $\mathrm{nR}$ & 1 & nonA & $\mathrm{S}$ \\
\hline NGC 4956 & 65.6 & 6.8 & 10.873 & -0.830 & -1.672 & E1 & A & $\mathrm{fR}$ & 1 & nonA & $\mathrm{S}$ \\
\hline NGC 4961 & 35.0 & 11.1 & 9.478 & 0.070 & 0.302 & Scd & B & SF & 1 & nonA & $\mathrm{S}$ \\
\hline NGC 5000 & 77.1 & 14.5 & 10.667 & 0.233 & -0.450 & Sbc & B & QnR & 2 & nonA & $\mathrm{S}$ \\
\hline NGC 5029 & 120.9 & 9.5 & 11.423 & -0.474 & -1.740 & E6 & $\mathrm{A}$ & $\mathrm{nR}$ & 1 & nonA & $\mathrm{S}$ \\
\hline NGC 5056 & 77.0 & 13.2 & - & - & - & Sc & $\mathrm{AB}$ & SF & 1 & nonA & $\mathrm{S}$ \\
\hline
\end{tabular}


Table B.1. continued.

\begin{tabular}{|c|c|c|c|c|c|c|c|c|c|c|c|}
\hline $\begin{array}{l}\text { Galaxy } \\
\text { (1) }\end{array}$ & $\begin{array}{c}D \\
\mathrm{Mpc} \\
(2) \\
\end{array}$ & $\begin{array}{c}\mathrm{Re} \\
\operatorname{arcsec} \\
(3) \\
\end{array}$ & $\begin{array}{c}\log M_{*} \\
M_{\odot} \\
(4)\end{array}$ & $\begin{array}{c}\log \mathrm{SFR} \\
M_{\odot} \mathrm{yr}^{-1} \\
(5)\end{array}$ & $\begin{array}{c}\Delta \mathrm{SFR} \\
M_{\odot} \mathrm{yr}^{-1} \\
(6)\end{array}$ & $\begin{array}{l}\text { Type } \\
(7) \\
\end{array}$ & $\begin{array}{l}\text { Bar } \\
(8) \\
\end{array}$ & $\begin{array}{l}\text { QS } \\
\text { (9) } \\
\end{array}$ & $\begin{array}{c}\text { flag-QS } \\
(10)\end{array}$ & $\begin{array}{l}\text { NA } \\
(11) \\
\end{array}$ & $\begin{array}{c}\text { flag-NA } \\
(12)\end{array}$ \\
\hline NGC 5218 & 41.9 & 16.1 & 10.445 & 0.083 & -0.429 & $\mathrm{Sab}$ & B & MX & 1 & nonA & S \\
\hline NGC 5378 & 42.7 & 16.6 & 10.341 & -0.976 & -1.408 & $\mathrm{Sb}$ & B & MX & 2 & nonA & S \\
\hline NGC 5480 & 27.0 & 15.1 & 9.716 & 0.172 & 0.221 & Scd & A & SF & 1 & nonA & $\mathrm{S}$ \\
\hline NGC 5485 & 28.1 & 12.8 & 10.530 & -1.780 & -2.358 & E5 & A & $\mathrm{fR}$ & 1 & nonA & $\mathrm{S}$ \\
\hline NGC 5520 & 27.3 & 11.0 & 9.691 & -0.041 & 0.027 & Sbc & A & SF & 1 & nonA & $\mathrm{S}$ \\
\hline NGC 5614 & 54.4 & 12.3 & 11.109 & -0.098 & -1.122 & $\mathrm{Sa}$ & $\mathrm{A}$ & $\mathrm{nR}$ & 1 & wAGN & $\mathrm{S}$ \\
\hline NGC 5630 & 37.7 & 13.3 & 9.513 & 0.284 & 0.490 & $\mathrm{Sdm}$ & B & SF & 1 & nonA & $\mathrm{S}$ \\
\hline NGC 5633 & 33.5 & 11.2 & 10.012 & 0.325 & 0.146 & $\mathrm{Sbc}$ & A & $\mathrm{SF}$ & 1 & nonA & $\mathrm{S}$ \\
\hline NGC 5657 & 54.5 & 10.7 & 10.260 & 0.190 & -0.179 & Sbc & $\mathrm{B}$ & SF & 1 & nonA & $\mathrm{S}$ \\
\hline NGC 5682 & 32.8 & 25.2 & 9.109 & -0.483 & 0.034 & Scd & B & SF & 1 & nonA & S \\
\hline NGC 5720 & 108.4 & 12.4 & 10.947 & 0.120 & -0.779 & $\mathrm{Sbc}$ & B & $\mathrm{cQ}$ & 1 & nonA & $\mathrm{S}$ \\
\hline NGC 5732 & 52.7 & 13.2 & 9.726 & 0.026 & 0.067 & Sbc & A & SF & 1 & nonA & $\mathrm{S}$ \\
\hline NGC 5784 & 75.1 & 8.6 & 11.054 & -0.068 & -1.048 & SO & $\mathrm{A}$ & $\mathrm{nR}$ & 1 & nonA & $\mathrm{S}$ \\
\hline NGC 5876 & 46.9 & 11.6 & 10.654 & -0.833 & -1.506 & SOa & B & $\mathrm{nR}$ & 2 & nonA & $\mathrm{S}$ \\
\hline NGC 5888 & 121.3 & 12.0 & 11.273 & 0.360 & -0.789 & $\mathrm{Sb}$ & B & $\mathrm{cQ}$ & 2 & nonA & $\mathrm{S}$ \\
\hline NGC 5908 & 47.3 & 16.2 & 10.941 & -0.051 & -0.945 & $\mathrm{Sa}$ & A & MX & 1 & nonA & $\mathrm{S}$ \\
\hline NGC 5971 & 61.2 & 9.8 & 10.079 & -0.421 & -0.651 & $\mathrm{Sb}$ & $\mathrm{AB}$ & MX & 2 & wAGN & $\mathrm{S}$ \\
\hline NGC 5980 & 57.1 & 13.2 & 10.666 & 0.848 & 0.166 & $\mathrm{Sbc}$ & $\mathrm{A}$ & SF & 1 & nonA & $\mathrm{S}$ \\
\hline NGC 5987 & 43.5 & 19.7 & 10.753 & -0.796 & -1.545 & $\mathrm{Sa}$ & A & $\mathrm{nR}$ & 1 & nonA & S \\
\hline NGC 6020 & 60.4 & 7.3 & 10.808 & -1.451 & -2.242 & $\mathrm{E} 4$ & $\mathrm{~A}$ & $\mathrm{fR}$ & 1 & nonA & $\mathrm{S}$ \\
\hline NGC 6021 & 66.2 & 5.9 & 10.896 & -1.282 & -2.142 & E5 & $\mathrm{A}$ & $\mathrm{fR}$ & 1 & nonA & $\mathrm{S}$ \\
\hline NGC 6032 & 60.1 & 14.8 & 10.281 & -0.297 & -0.683 & $\mathrm{Sbc}$ & $\mathrm{B}$ & MX & 2 & nonA & $\mathrm{S}$ \\
\hline NGC 6060 & 62.3 & 22.3 & 10.795 & 0.676 & -0.106 & $\mathrm{Sb}$ & $\mathrm{A}$ & SF & 1 & nonA & $\mathrm{S}$ \\
\hline NGC 6063 & 40.0 & 17.9 & 9.924 & -0.372 & -0.483 & $\mathrm{Sbc}$ & A & SF & 1 & nonA & $\mathrm{S}$ \\
\hline NGC 6081 & 72.0 & 10.0 & 10.935 & -0.546 & -1.435 & SOa & A & $\mathrm{nR}$ & 1 & sAGN & $\mathrm{U}$ \\
\hline NGC 6125 & 68.6 & 8.0 & 11.202 & -1.426 & -2.521 & E1 & A & $\mathrm{fR}$ & 1 & nonA & $\mathrm{S}$ \\
\hline NGC 6132 & 69.2 & 14.2 & 10.099 & 0.325 & 0.080 & Sbc & A & SF & 1 & nonA & $\mathrm{S}$ \\
\hline NGC 6146 & 123.0 & 7.9 & 11.463 & -0.767 & -2.063 & E5 & A & $\mathrm{nR}$ & 1 & wAGN & $\mathrm{U}$ \\
\hline NGC 6150 & 121.6 & 7.2 & 11.252 & -0.744 & -1.878 & E7 & $\mathrm{A}$ & $\mathrm{fR}$ & 1 & nonA & $\mathrm{S}$ \\
\hline NGC 6168 & 36.1 & 18.1 & 9.596 & -0.031 & 0.111 & $\mathrm{Sc}$ & $\mathrm{AB}$ & SF & 1 & nonA & $\mathrm{S}$ \\
\hline NGC 6173 & 122.5 & 13.9 & 11.681 & -0.576 & -2.040 & E6 & $\mathrm{A}$ & $\mathrm{nR}$ & 2 & nonA & $\mathrm{S}$ \\
\hline NGC 6186 & 41.9 & 11.9 & 10.454 & 0.183 & -0.336 & $\mathrm{Sb}$ & B & MX & 1 & nonA & $\mathrm{S}$ \\
\hline NGC 6278 & 40.7 & 8.4 & 10.676 & -1.970 & -2.660 & SOa & $\mathrm{AB}$ & $\mathrm{fR}$ & 1 & nonA & $\mathrm{S}$ \\
\hline NGC 6301 & 116.8 & 21.5 & 10.870 & 0.524 & -0.315 & $\mathrm{Sbc}$ & A & SF & 1 & nonA & $\mathrm{S}$ \\
\hline NGC 6310 & 49.5 & 15.3 & 10.451 & -0.353 & -0.870 & $\mathrm{Sb}$ & A & $\mathrm{cQ}$ & 1 & nonA & $\mathrm{S}$ \\
\hline NGC 6314 & 92.9 & 9.4 & 10.921 & -0.195 & -1.073 & $\mathrm{Sab}$ & $\mathrm{A}$ & $\mathrm{nR}$ & 1 & nonA & $\mathrm{S}$ \\
\hline NGC 6478 & 95.6 & 15.5 & 10.986 & 0.796 & -0.133 & $\mathrm{Sc}$ & A & SF & 1 & nonA & $\mathrm{S}$ \\
\hline NGC 6497 & 87.2 & 12.3 & 10.885 & -0.050 & -0.901 & $\mathrm{Sab}$ & B & $\mathrm{cQ}$ & 1 & nonA & $\mathrm{S}$ \\
\hline NGC 6515 & 96.7 & 10.8 & 11.086 & -0.945 & -1.951 & E3 & $\mathrm{A}$ & $\mathrm{nR}$ & 1 & nonA & $\mathrm{S}$ \\
\hline NGC 6762 & 43.0 & 10.3 & 10.205 & -1.714 & -2.042 & $\mathrm{Sab}$ & A & $\mathrm{nR}$ & 1 & wAGN & $\mathrm{S}$ \\
\hline NGC 6941 & 87.2 & 16.6 & 10.982 & 0.072 & -0.853 & $\mathrm{Sb}$ & B & QnR & 1 & nonA & $\mathrm{S}$ \\
\hline NGC 6945 & 53.9 & 8.8 & 9.385 & -2.882 & -2.578 & So & B & fR & 1 & nonA & $\mathrm{S}$ \\
\hline NGC 6978 & 84.5 & 14.9 & 10.814 & 0.198 & -0.598 & $\mathrm{Sb}$ & $\mathrm{AB}$ & MX & 2 & nonA & $\mathrm{S}$ \\
\hline NGC 7025 & 70.8 & 13.0 & 11.242 & -0.629 & -1.754 & SOa & A & $\mathrm{nR}$ & 1 & nonA & $\mathrm{S}$ \\
\hline NGC 7047 & 81.7 & 17.3 & 10.721 & 0.324 & -0.401 & $\mathrm{Sbc}$ & $\mathrm{B}$ & $\mathrm{cQ}$ & 1 & nonA & $\mathrm{S}$ \\
\hline NGC 7194 & 112.7 & 3.6 & 11.345 & -1.158 & -2.363 & E3 & A & fR & 1 & nonA & $\mathrm{S}$ \\
\hline NGC 7311 & 64.3 & 8.6 & 11.117 & 0.105 & -0.925 & $\mathrm{Sa}$ & $\mathrm{A}$ & $\mathrm{cQ}$ & 1 & nonA & $\mathrm{S}$ \\
\hline NGC 7321 & 100.5 & 13.0 & 11.028 & 0.495 & -0.466 & Sbc & B & QnR & 1 & wAGN & $\mathrm{U}$ \\
\hline NGC 7364 & 68.5 & 8.0 & 10.706 & 0.483 & -0.230 & $\mathrm{Sab}$ & $\mathrm{A}$ & SF & 1 & nonA & $\mathrm{S}$ \\
\hline NGC 7466 & 105.5 & 12.7 & 10.709 & 0.455 & -0.260 & Sbc & $\mathrm{A}$ & SF & 1 & sAGN & $\mathrm{S}$ \\
\hline NGC 7489 & 88.0 & 16.2 & 10.493 & 0.748 & 0.199 & Sbc & $\mathrm{A}$ & SF & 1 & nonA & $\mathrm{S}$ \\
\hline NGC 7549 & 67.3 & 19.1 & 10.510 & 0.780 & 0.218 & $\mathrm{Sbc}$ & $\mathrm{B}$ & SF & 2 & nonA & $\mathrm{S}$ \\
\hline NGC 7550 & 71.9 & 11.2 & 11.157 & -1.067 & -2.128 & $\mathrm{E} 4$ & $\mathrm{~A}$ & $\mathrm{nR}$ & 1 & wAGN & $\mathrm{U}$ \\
\hline NGC 7562 & 51.4 & 10.5 & 11.187 & -0.979 & -2.063 & $\mathrm{E} 4$ & $\mathrm{~A}$ & $\mathrm{fR}$ & 1 & nonA & $\mathrm{S}$ \\
\hline NGC 7563 & 59.4 & 7.5 & 11.026 & -1.391 & -2.350 & $\mathrm{Sa}$ & B & $\mathrm{fR}$ & 1 & nonA & $\mathrm{S}$ \\
\hline NGC 7591 & 69.9 & 11.7 & 10.731 & 0.703 & -0.030 & $\mathrm{Sbc}$ & B & SF & 1 & nonA & $\mathrm{S}$ \\
\hline
\end{tabular}


Table B.1. continued.

\begin{tabular}{|c|c|c|c|c|c|c|c|c|c|c|c|}
\hline $\begin{array}{l}\text { Galaxy } \\
\text { (1) }\end{array}$ & $\begin{array}{c}D \\
\mathrm{Mpc} \\
(2) \\
\end{array}$ & $\begin{array}{c}\mathrm{Re} \\
\operatorname{arcsec} \\
(3) \\
\end{array}$ & $\begin{array}{c}\log M_{*} \\
M_{\odot} \\
(4)\end{array}$ & $\begin{array}{c}\log \mathrm{SFR} \\
M_{\odot} \mathrm{yr}^{-1} \\
(5)\end{array}$ & $\begin{array}{c}\Delta \mathrm{SFR} \\
M_{\odot} \mathrm{yr}^{-1} \\
(6)\end{array}$ & $\begin{array}{l}\text { Type } \\
(7) \\
\end{array}$ & $\begin{array}{l}\text { Bar } \\
(8) \\
\end{array}$ & $\begin{array}{l}\text { QS } \\
\text { (9) } \\
\end{array}$ & $\begin{array}{c}\text { flag-QS } \\
(10)\end{array}$ & $\begin{array}{l}\text { NA } \\
\text { (11) } \\
\end{array}$ & $\begin{array}{c}\text { flag-NA } \\
\text { (12) }\end{array}$ \\
\hline NGC 7608 & 50.1 & 12.4 & 9.946 & 0.043 & -0.085 & $\mathrm{Sbc}$ & A & $\mathrm{SF}$ & 1 & nonA & S \\
\hline NGC 7611 & 46.6 & 9.0 & 10.652 & -0.999 & -1.671 & So & A & $\mathrm{fR}$ & 1 & nonA & $\mathrm{S}$ \\
\hline NGC 7619 & 53.6 & 11.2 & 11.267 & -1.075 & -2.220 & E3 & A & $\mathrm{fR}$ & 1 & nonA & $\mathrm{S}$ \\
\hline NGC 7623 & 53.3 & 6.8 & 10.732 & -1.353 & -2.087 & So & A & $\mathrm{fR}$ & 1 & nonA & $\mathrm{S}$ \\
\hline NGC 7625 & 24.7 & 10.7 & 9.963 & 0.210 & 0.069 & $\mathrm{Sa}$ & A & SF & 2 & nonA & $\mathrm{S}$ \\
\hline NGC 7631 & 53.5 & 13.8 & 10.507 & 0.009 & -0.551 & $\mathrm{Sb}$ & A & QnR & 1 & nonA & $S$ \\
\hline NGC 7653 & 60.7 & 11.8 & 10.512 & 0.471 & -0.093 & $\mathrm{Sb}$ & A & $\mathrm{cQ}$ & 1 & nonA & $\mathrm{S}$ \\
\hline NGC 7671 & 58.7 & 6.4 & 10.798 & -2.010 & -2.794 & S0 & A & $\mathrm{fR}$ & 1 & nonA & $\mathrm{S}$ \\
\hline NGC 7683 & 53.2 & 9.6 & 10.972 & -1.129 & -2.048 & So & $\mathrm{A}$ & $\mathrm{fR}$ & 1 & nonA & $\mathrm{S}$ \\
\hline NGC 7711 & 57.8 & 12.0 & 10.835 & -1.047 & -1.860 & E7 & A & MX & 2 & nonA & $\mathrm{S}$ \\
\hline NGC 7716 & 37.0 & 12.3 & 10.410 & -0.231 & -0.716 & $\mathrm{Sb}$ & A & $\mathrm{cQ}$ & 1 & nonA & $\mathrm{S}$ \\
\hline NGC 7722 & 57.4 & 12.9 & 10.934 & 0.085 & -0.804 & $\mathrm{Sab}$ & A & MX & 2 & nonA & $\mathrm{S}$ \\
\hline NGC 7738 & 94.2 & 13.1 & 11.031 & 0.716 & -0.247 & $\mathrm{Sb}$ & $\mathrm{B}$ & MX & 1 & nonA & $\mathrm{S}$ \\
\hline NGC 7787 & 92.8 & 11.2 & 10.573 & 0.215 & -0.396 & $\mathrm{Sab}$ & $\mathrm{AB}$ & QnR & 1 & nonA & $\mathrm{S}$ \\
\hline NGC 7819 & 70.4 & 12.4 & 10.068 & 0.326 & 0.104 & $\mathrm{Sc}$ & $\mathrm{A}$ & SF & 1 & nonA & $S$ \\
\hline NGC 7824 & 85.7 & 9.6 & 11.045 & -0.224 & -1.198 & $\mathrm{Sab}$ & $\mathrm{A}$ & $\mathrm{nR}$ & 1 & sAGN & $\mathrm{U}$ \\
\hline UGC00005 & 101.0 & 13.0 & 10.843 & 0.795 & -0.023 & Sbc & A & $\mathrm{SF}$ & 1 & sAGN & $\mathrm{U}$ \\
\hline UGC00029 & 122.7 & 10.4 & - & - & - & E1 & $\mathrm{A}$ & $\mathrm{nR}$ & 1 & nonA & $\mathrm{S}$ \\
\hline UGC00036 & 87.9 & 11.2 & 10.897 & -0.083 & -0.943 & $\mathrm{Sab}$ & $\mathrm{AB}$ & MX & 1 & nonA & $\mathrm{S}$ \\
\hline UGC00148 & 59.7 & 4.5 & 10.148 & 0.831 & 0.547 & $\mathrm{Sc}$ & $\mathrm{A}$ & SF & 1 & nonA & $S$ \\
\hline UGC00312 & 61.4 & 15.7 & 9.851 & 0.439 & 0.384 & $\mathrm{Sd}$ & $\mathrm{B}$ & SF & 1 & nonA & $\mathrm{S}$ \\
\hline UGC00809 & 59.6 & 11.7 & 9.586 & -0.107 & 0.042 & Scd & $\mathrm{A}$ & SF & 1 & nonA & $\mathrm{S}$ \\
\hline UGC00987 & 65.8 & 9.8 & 10.724 & -0.303 & -1.030 & $\mathrm{Sa}$ & $\mathrm{AB}$ & SF & 1 & sAGN & $\mathrm{S}$ \\
\hline UGC01057 & 88.7 & 10.8 & 10.258 & 0.328 & -0.040 & $\mathrm{Sc}$ & $\mathrm{AB}$ & SF & 1 & nonA & $S$ \\
\hline UGC01271 & 70.8 & 6.1 & 10.772 & -0.724 & -1.487 & SOa & B & $\mathrm{fR}$ & 1 & nonA & $\mathrm{S}$ \\
\hline UGC02222 & 70.6 & 10.3 & 10.696 & -1.237 & -1.942 & SOa & $\mathrm{AB}$ & $\mathrm{fR}$ & 1 & nonA & $S$ \\
\hline UGC02229 & 100.5 & 16.3 & 10.918 & -0.544 & -1.420 & SOa & $\mathrm{A}$ & $\mathrm{nR}$ & 1 & nonA & $\mathrm{S}$ \\
\hline UGC02403 & 56.8 & 12.3 & 10.395 & 0.153 & -0.320 & $\mathrm{Sb}$ & B & SF & 1 & nonA & $\mathrm{S}$ \\
\hline UGC03253 & 58.8 & 19.2 & 10.360 & -0.181 & -0.628 & $\mathrm{Sb}$ & $\mathrm{B}$ & QnR & 1 & nonA & $\mathrm{S}$ \\
\hline UGC03539 & 46.7 & 19.2 & 9.654 & -0.072 & 0.025 & $\mathrm{Sc}$ & $\mathrm{AB}$ & SF & 1 & nonA & $\mathrm{S}$ \\
\hline UGC03995 & 64.4 & 18.0 & 10.933 & -0.003 & -0.891 & $\mathrm{Sb}$ & B & QnR & 2 & sAGN & $\mathrm{S}$ \\
\hline UGC04029 & 60.1 & 17.9 & 10.250 & 0.148 & -0.214 & $\mathrm{Sc}$ & $\mathrm{AB}$ & SF & 1 & nonA & $\mathrm{S}$ \\
\hline UGC04132 & 71.0 & 14.3 & 10.748 & 0.893 & 0.147 & $\mathrm{Sbc}$ & $\mathrm{AB}$ & SF & 1 & nonA & $\mathrm{S}$ \\
\hline UGC04145 & 62.0 & 8.6 & 10.468 & 0.509 & -0.021 & $\mathrm{Sa}$ & $\mathrm{AB}$ & MX & 1 & sAGN & $S$ \\
\hline UGC04197 & 61.1 & 15.6 & 10.497 & -0.342 & -0.894 & $\mathrm{Sab}$ & $\mathrm{AB}$ & $\mathrm{cQ}$ & 1 & nonA & $\mathrm{S}$ \\
\hline UGC04280 & 48.8 & 9.9 & 10.190 & -0.318 & -0.634 & $\mathrm{Sb}$ & $\mathrm{AB}$ & SF & 1 & nonA & $\mathrm{S}$ \\
\hline UGC04308 & 47.8 & 20.6 & 10.021 & 0.191 & 0.006 & $\mathrm{Sc}$ & $\mathrm{B}$ & SF & 1 & nonA & $\mathrm{S}$ \\
\hline UGC05108 & 110.2 & 12.9 & 10.927 & 0.164 & -0.719 & $\mathrm{Sb}$ & $\mathrm{B}$ & QnR & 1 & nonA & $\mathrm{S}$ \\
\hline UGC05113 & 94.7 & 8.1 & 10.913 & -1.213 & -2.085 & SOa & $\mathrm{AB}$ & $\mathrm{nR}$ & 1 & nonA & $\mathrm{S}$ \\
\hline UGC05598 & 76.2 & 11.9 & 10.066 & 0.219 & -0.001 & $\mathrm{Sb}$ & $\mathrm{A}$ & SF & 1 & nonA & $S$ \\
\hline UGC05771 & 101.6 & 6.9 & 11.141 & -0.318 & -1.366 & E6 & A & $\mathrm{nR}$ & 1 & nonA & $\mathrm{S}$ \\
\hline UGC05990 & 21.3 & 7.7 & - & - & - & $\mathrm{Sc}$ & A & SF & 1 & nonA & $\mathrm{S}$ \\
\hline UGC06036 & 89.0 & 10.2 & 11.039 & -0.324 & -1.293 & $\mathrm{Sa}$ & A & MX & 1 & nonA & $\mathrm{S}$ \\
\hline UGC06312 & 85.2 & 11.5 & 10.793 & -0.161 & -0.941 & $\mathrm{Sab}$ & A & MX & 1 & nonA & $\mathrm{S}$ \\
\hline UGC07012 & 42.1 & 10.4 & 9.266 & -0.175 & 0.220 & Scd & $\mathrm{AB}$ & $\mathrm{SF}$ & 1 & nonA & $S$ \\
\hline UGC07145 & 91.3 & 12.4 & 10.343 & 0.059 & -0.374 & $\mathrm{Sbc}$ & $\mathrm{A}$ & SF & 1 & nonA & $\mathrm{S}$ \\
\hline UGC08107 & 115.0 & 15.4 & 11.059 & 0.834 & -0.151 & $\mathrm{Sa}$ & $\mathrm{A}$ & SF & 1 & nonA & $\mathrm{S}$ \\
\hline UGC08231 & 35.2 & 13.9 & 9.035 & -0.216 & 0.358 & $\mathrm{Sd}$ & $\mathrm{AB}$ & SF & 1 & nonA & $\mathrm{S}$ \\
\hline UGC08234 & 112.6 & 4.7 & 11.040 & 0.070 & -0.900 & So & $\mathrm{A}$ & $\mathrm{fR}$ & 1 & nonA & $\mathrm{S}$ \\
\hline UGC08733 & 33.2 & 19.8 & 9.072 & -0.788 & -0.243 & $\mathrm{Sdm}$ & $\mathrm{B}$ & SF & 1 & nonA & $\mathrm{S}$ \\
\hline UGC08778 & 45.8 & 9.8 & 9.994 & -0.594 & -0.759 & $\mathrm{Sb}$ & A & MX & 1 & nonA & $\mathrm{S}$ \\
\hline UGC08781 & 104.3 & 10.9 & 10.978 & -0.138 & -1.060 & $\mathrm{Sb}$ & B & $\mathrm{cQ}$ & 1 & nonA & $S$ \\
\hline UGC09476 & 46.2 & 17.2 & 10.032 & 0.195 & 0.001 & $\mathrm{Sbc}$ & A & SF & 1 & nonA & $\mathrm{S}$ \\
\hline UGC09537 & 122.1 & 16.5 & 11.054 & 0.212 & -0.770 & $\mathrm{Sb}$ & A & $\mathrm{cQ}$ & 1 & nonA & $\mathrm{S}$ \\
\hline UGC09542 & 76.4 & 15.0 & 10.185 & 0.227 & -0.085 & $\mathrm{Sc}$ & $\mathrm{A}$ & $\mathrm{SF}$ & 1 & nonA & $\mathrm{S}$ \\
\hline UGC09665 & 36.8 & 14.5 & 9.798 & 0.069 & 0.055 & $\mathrm{Sb}$ & A & SF & 1 & nonA & $S$ \\
\hline UGC09873 & 78.8 & 16.3 & 9.905 & -0.076 & -0.172 & $\mathrm{Sb}$ & $\mathrm{A}$ & SF & 1 & nonA & $\mathrm{S}$ \\
\hline
\end{tabular}


Table B.1. continued.

\begin{tabular}{|c|c|c|c|c|c|c|c|c|c|c|c|}
\hline $\begin{array}{l}\text { Galaxy } \\
\text { (1) }\end{array}$ & $\begin{array}{c}D \\
\mathrm{Mpc} \\
(2) \\
\end{array}$ & $\begin{array}{c}\mathrm{Re} \\
\operatorname{arcsec} \\
(3) \\
\end{array}$ & $\begin{array}{c}\log M_{*} \\
M_{\odot} \\
(4)\end{array}$ & $\begin{array}{c}\log \mathrm{SFR} \\
M_{\odot} \mathrm{yr}^{-1} \\
(5)\end{array}$ & $\begin{array}{c}\Delta \mathrm{SFR} \\
M_{\odot} \mathrm{yr}^{-1} \\
(6)\end{array}$ & $\begin{array}{c}\text { Type } \\
(7)\end{array}$ & $\begin{array}{l}\text { Bar } \\
(8) \\
\end{array}$ & $\begin{array}{l}\text { QS } \\
(9) \\
\end{array}$ & $\begin{array}{c}\text { flag-QS } \\
(10)\end{array}$ & $\begin{array}{l}\text { NA } \\
(11) \\
\end{array}$ & $\begin{array}{c}\text { flag-NA } \\
(12)\end{array}$ \\
\hline UGC09892 & 79.5 & 13.0 & 10.137 & 0.165 & -0.110 & $\mathrm{Sbc}$ & A & SF & 1 & nonA & $\mathrm{S}$ \\
\hline UGC10097 & 83.8 & 7.8 & 11.242 & -0.973 & -2.099 & E5 & A & $\mathrm{nR}$ & 1 & nonA & $\mathrm{S}$ \\
\hline UGC10123 & 53.8 & 10.6 & 10.306 & 0.328 & -0.077 & $\mathrm{Sab}$ & A & $\mathrm{SF}$ & 1 & nonA & $\mathrm{S}$ \\
\hline UGC10205 & 91.6 & 14.6 & 10.952 & 0.353 & -0.550 & $\mathrm{~S} 0 \mathrm{a}$ & $\mathrm{A}$ & $\mathrm{nR}$ & 1 & nonA & $\mathrm{S}$ \\
\hline UGC10257 & 54.5 & 20.1 & 10.511 & 0.858 & 0.294 & $\mathrm{Sbc}$ & A & SF & 1 & nonA & $\mathrm{S}$ \\
\hline UGC10331 & 63.7 & 17.3 & 9.840 & 0.184 & 0.138 & $\mathrm{Sc}$ & $\mathrm{AB}$ & $\mathrm{SF}$ & 1 & nonA & $\mathrm{S}$ \\
\hline UGC10337 & 121.1 & 11.3 & 10.967 & 0.540 & -0.375 & $\mathrm{Sb}$ & $\mathrm{A}$ & $\mathrm{cQ}$ & 1 & nonA & $\mathrm{S}$ \\
\hline UGC10384 & 69.3 & 8.6 & 10.234 & 0.639 & 0.290 & $\mathrm{Sb}$ & A & $\mathrm{SF}$ & 1 & nonA & $\mathrm{S}$ \\
\hline UGC10388 & 64.9 & 6.7 & 10.473 & -0.718 & -1.252 & $\mathrm{Sa}$ & $\mathrm{AB}$ & MX & 2 & nonA & $\mathrm{S}$ \\
\hline UGC10650 & 42.5 & 11.3 & 9.170 & -0.218 & 0.252 & Scd & A & SF & 1 & nonA & $\mathrm{S}$ \\
\hline UGC10693 & 116.9 & 10.2 & 11.272 & -0.655 & -1.804 & E7 & $\mathrm{AB}$ & $\mathrm{fR}$ & 1 & nonA & $\mathrm{S}$ \\
\hline UGC10695 & 116.5 & 11.7 & 11.190 & -0.733 & -1.819 & E5 & A & $\mathrm{nR}$ & 1 & nonA & $\mathrm{S}$ \\
\hline UGC10710 & 117.3 & 13.9 & 10.885 & 1.275 & 0.424 & $\mathrm{Sb}$ & A & SF & 1 & non $A$ & $\mathrm{~S}$ \\
\hline UGC10796 & 44.8 & 11.4 & 9.199 & -0.259 & 0.188 & Scd & $\mathrm{AB}$ & SF & 1 & nonA & $\mathrm{S}$ \\
\hline UGC10811 & 122.5 & 10.7 & 10.955 & 0.468 & -0.437 & $\mathrm{Sb}$ & B & $\mathrm{cQ}$ & 1 & nonA & $\mathrm{S}$ \\
\hline UGC10905 & 109.7 & 17.6 & 11.277 & -0.342 & -1.495 & SOa & $\mathrm{A}$ & $\mathrm{nR}$ & 1 & nonA & $\mathrm{S}$ \\
\hline UGC10972 & 66.1 & 15.0 & 10.329 & -0.219 & -0.642 & $\mathrm{Sbc}$ & $\mathrm{A}$ & $\mathrm{cQ}$ & 1 & nonA & $\mathrm{S}$ \\
\hline UGC11228 & 81.9 & 6.2 & 10.962 & -1.303 & -2.214 & So & B & $\mathrm{nR}$ & 1 & nonA & $\mathrm{S}$ \\
\hline UGC11717 & 89.1 & 11.6 & 10.824 & 0.802 & -0.001 & $\mathrm{Sab}$ & A & MX & 1 & sAGN & $\mathrm{U}$ \\
\hline UGC12054 & 30.6 & 10.0 & 8.993 & -0.557 & 0.048 & $\mathrm{Sc}$ & $\mathrm{A}$ & SF & 1 & nonA & $\mathrm{S}$ \\
\hline UGC12127 & 116.2 & 16.5 & 11.339 & -0.702 & -1.903 & E1 & $\mathrm{A}$ & $\mathrm{nR}$ & 1 & nonA & $\mathrm{S}$ \\
\hline UGC12185 & 93.9 & 16.5 & 10.693 & -0.128 & -0.831 & $\mathrm{Sb}$ & B & $\mathrm{cQ}$ & 1 & non $A$ & $\mathrm{~S}$ \\
\hline UGC12274 & 107.7 & 20.6 & 11.072 & -0.340 & -1.336 & $\mathrm{Sa}$ & $\mathrm{A}$ & $\mathrm{nR}$ & 1 & nonA & $\mathrm{S}$ \\
\hline UGC12308 & 32.9 & 20.3 & 8.916 & -0.571 & 0.094 & Scd & $\mathrm{A}$ & $\mathrm{SF}$ & 1 & nonA & $\mathrm{S}$ \\
\hline UGC12518 & 40.2 & 11.8 & 10.374 & -0.490 & -0.948 & $\mathrm{Sb}$ & $\mathrm{A}$ & MX & 2 & nonA & $\mathrm{S}$ \\
\hline UGC12519 & 62.3 & 12.5 & 10.124 & 0.325 & 0.060 & $\mathrm{Sc}$ & $\mathrm{AB}$ & SF & 1 & nonA & $\mathrm{S}$ \\
\hline UGC12723 & 76.9 & 5.8 & 9.628 & -0.144 & -0.027 & $\mathrm{Sc}$ & $\mathrm{A}$ & SF & 1 & nonA & $\mathrm{S}$ \\
\hline UGC12857 & 35.5 & 13.8 & 9.578 & -0.213 & -0.058 & Sbc & A & $\mathrm{SF}$ & 1 & nonA & $\mathrm{S}$ \\
\hline
\end{tabular}

Table B.2. Median values of galaxy properties from the box-and-whisker diagrams in Fig. 9 (see Sects. 2 and 5).

\begin{tabular}{|c|c|c|c|c|c|c|c|c|c|c|c|c|}
\hline QS & $B / D$ & $\begin{array}{l}\log L_{\mathrm{r}}^{\text {tot }} \\
{\left[L_{\odot}\right]} \\
(3)\end{array}$ & $\begin{array}{l}R_{\mathrm{e}} \\
{[\mathrm{kpc}]} \\
(4)\end{array}$ & $\begin{array}{l}\log \mu_{*} \\
{\left[M_{\odot} / \mathrm{pc}^{2}\right]} \\
(5)\end{array}$ & $\begin{array}{l}\log M_{*}^{\text {tot }} \\
{\left[M_{\odot}\right]} \\
(6)\end{array}$ & $\begin{array}{l}\log \tau \\
{[y r]} \\
(7) \\
\end{array}$ & $\begin{array}{l}\log Z \\
{\left[Z_{\odot}\right]} \\
(8) \\
\end{array}$ & $\begin{array}{l}\log \mathrm{SFR} \\
{\left[M_{\odot} \mathrm{yr}^{-1}\right]} \\
(9)\end{array}$ & $\begin{array}{l}\lambda_{R_{\mathrm{e}}} \\
(10) \\
\end{array}$ & $\begin{array}{l}V_{\mathrm{c}, \max } \\
{\left[\mathrm{km} \mathrm{s}^{-1}\right]} \\
(11)\end{array}$ & $\begin{array}{l}\log M_{\mathrm{dyn}}^{\mathrm{tot}} \\
(12)\end{array}$ & $\begin{array}{l}f_{\mathrm{d}} \\
(13)\end{array}$ \\
\hline $\mathrm{SF}$ & 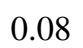 & 1 & & 2.38 & 10 & 8.80 & -0.24 & 0.20 & 0 & 17 & 7 & 0.80 \\
\hline QnR-nonA & 0.09 & 26 & 5. & 2.67 & 10.57 & 9.45 & 0.04 & 0.18 & 0.70 & 83 & 10 & 0.64 \\
\hline cQ-nonA & 0.11 & 10.51 & 4.81 & 2.83 & 10.64 & 9.52 & 0.12 & -0.03 & 0.66 & 237.26 & 11.18 & 0.65 \\
\hline MX-nonA & 0.30 & 10.50 & 3.89 & 3.01 & 10.80 & 9.65 & 0.08 & -0.15 & 0.58 & 245.28 & 11.25 & 0.66 \\
\hline nR-nonA & 0.58 & 10.79 & 4.89 & 3.19 & 11.07 & 9.81 & 0.19 & -0.63 & 0.29 & 343.61 & 11.62 & 0.68 \\
\hline fR-nonA & 0.69 & 10.47 & 2.23 & 3.39 & 10.81 & 9.77 & 0.21 & -1.25 & 0.39 & 326.74 & 11.22 & 0.64 \\
\hline
\end{tabular}

Notes. Columns list: (1) QS of non-active (nonA) galaxies, where: SF - Star-forming, QnR - quiescent-nuclear-ring; cQ - centrally quiescent; MX - mixed; $\mathrm{nR}$ - nearly retired and fR - fully retired (Sect. 3); (2) The median bulge-to-disc ratios for a given EL class; (3) The median value of the total $r$-band luminosity for a given EL class; (4) The median values of the effective radius for a given EL class; (5) The median stellar mass surface density for a given EL class; (6) The median total stellar mass of the galaxies for a given EL class; (7) The median value of the galaxy age for a given EL class; (8) The median value of galaxy metallicity for a given EL class; (9) The median value of star formation rate for a given EL class; (10) The median value of the specific angular momentum within one effective radius for a given EL class; (11) The median value of the maximum circular velocity for a given EL class; (12) The median value of the total dynamical mass for a given EL class; (13) The median value of mass discrepancy factor, $f_{\mathrm{d}}=1-\left(M_{*}^{\text {tot }} / M_{\mathrm{dyn}}^{\text {tot }}\right)$, for a given EL class; see text in Sects. 2 and 5 for more details. 